\title{
Simulations of starspot anomalies within TESS exoplanetary transit light curves
}

\section{Detection limits of starspot anomalies in TESS light curves $\star$}

\author{
J. Tregloan-Reed and E. Unda-Sanzana
}

\begin{abstract}
Centro de Astronomía (CITEVA), Universidad de Antofagasta, Avenida U. de Antofagasta 02800, Antofagasta, Chile e-mail: jeremy.tregloanreed@uantof.cl
\end{abstract}

Received 19 April 2019 / Accepted 14 August 2019

\begin{abstract}
Context. The primary targets of the NASA Transiting Exoplanet Survey Satellite (TESS) are K and M dwarf stars within our solar neighbourhood. Young $\mathrm{K}$ and $\mathrm{M}$ dwarf stars are known to exhibit a high starspot coverage $(\approx 50 \%)$, however, older stars are known to show fewer starspots. This implies that TESS transit light curves at 2 min cadence may contain starspot anomalies, and if so, will require transit-starspot models to determine accurately the properties of the system.

Aims. The goals are to determine if starspot anomalies can manifest in TESS transit light curves, to determine the detection limits of the starspot anomalies, and to examine the relationship between the change in flux caused by the starspot anomaly and the planetary transit.

Methods. We conducted 20573 simulations of planetary transits around spotted stars using the transit-starspot model, PRISM. In total 3888 different scenarios were considered using three different host star spectral types, M4V, M1V, and K5V. The mean amplitude of the starspot anomaly was measured and compared to the photometric precision of the light curve to determine if the characteristic "blip" of the starspot anomaly was noticeable in the light curve.

Results. The simulations show that starspot anomalies are observable in TESS 2 min cadence data. The smallest starspot detectable in TESS transit light curves has a radius of $\approx 1900 \mathrm{~km}$. The starspot detection limits for the three host stars are $4900 \pm 1700 \mathrm{~km}(\mathrm{M} 4 \mathrm{~V})$, $13800 \pm 6000 \mathrm{~km}(\mathrm{M} 1 \mathrm{~V})$, and $15900 \pm 6800 \mathrm{~km}(\mathrm{~K} 5 \mathrm{~V})$. The smallest change in flux of the starspot $\left(\Delta F_{\text {spot }}=0.00015 \pm 0.00001\right) \mathrm{can}$ be detected when the ratio of planetary to stellar radii $k=0.082 \pm 0.004$.

Conclusions. The results confirm known dependencies between the amplitude of the starspot anomaly and the photometric parameters of the light curve. The results facilitated the characterisation of the relationship between the change in flux of the starspot anomaly and the change in flux of the planetary transit for TESS transit light curves.
\end{abstract}

Key words. stars: late-type - stars: activity - starspots - planets and satellites: general - methods: numerical techniques: photometric

\section{Introduction}

The next generation of transit planet hunters (e.g. Next Generation Transit Survey, NGTS: Wheatley et al. 2013; NASA Transiting Exoplanet Survey Satellite, TESS: Ricker et al. 2009, 2014, 2015) have been observing transiting planets orbiting both $\mathrm{K}$ and $\mathrm{M}$ dwarf stars within the solar neighbourhood; these surveys have an emphasis on super-Earth: $1.25 R_{\oplus}$ to $2 R_{\oplus}$; subNeptune: $2 R_{\oplus}$ to $4 R_{\oplus}$; Neptune: $4 R_{\oplus}$ to $6 R_{\oplus}$ class planets. TiO absorption bands show that young $\mathrm{K}$ dwarfs can have $20-40 \%$ starspot coverage (O'Neal et al. 2004), while young M dwarfs can have a starspot coverage of $40 \pm 10 \%$ (Jackson \& Jeffries 2013). Older stars are known to show fewer starspots, however, the dependence on age has not been reliably quantified. This leads to the potential of an increased likelihood that the transits observed by these new planet hunters contain starspot anomalies.

Late-type stars $(\mathrm{G}, \mathrm{K}, \mathrm{M})$ have magnetic fields which often manifest themselves as dark spots on the stellar surface. When a transiting planet passes in front of a starspot, a short-term

\footnotetext{
* Simulated light curves are only available at the CDS via anonymous ftp to cdsarc.u-strasbg. fr (130.79.128.5) or via http:// cdsarc.u-strasbg.fr/viz-bin/cat/J/A+A/630/A114
}

increase in the brightness of the star is seen because the planet is temporarily blocking a fainter part of the surface of the star (Silva 2003). This "blip" in the light curve can be modelled to determine the size and position of the starspot (e.g. WASP-19: Tregloan-Reed et al. 2013; Mancini et al. 2013; WASP-6: Tregloan-Reed et al. 2015; CoRoT-2: Silva-Valio et al. 2010; WASP-4: Sanchis-Ojeda et al. 2011; HAT-P-11: SanchisOjeda \& Winn 2011; Kepler-30: Sanchis-Ojeda et al. 2012; Kepler-63: Sanchis-Ojeda et al. 2013; HATS-2: Mohler-Fischer et al. 2013; Qatar-2: Mancini et al. 2014; WASP-41: Southworth et al. 2016; WASP-52: Mancini et al. 2017; GJ 3470: Chen et al. 2017: Kepler-17: Valio et al. 2017).

Starspots can affect the shape of the light curve (Silva-Válio 2010) and if not correctly modelled can lead to biased measurements of the system parameters (e.g. planetary radius: Nikolov et al. 2013; limb darkening coefficients: Ballerini et al. 2012; time of minimum light: Sanchis-Ojeda et al. 2011). However, an accurate measurement of the planetary radius is achievable if the perturbations are accounted for, thereby improving investigations into the atmosphere, structure, and evolution of a planet (Fortney et al. 2007).

When two closely spaced transit light curves, both of which contain a starspot anomaly (due to the same starspot), 
are accurately modelled, the position of the starspot can be determined at two distinct points in time, allowing for an extraction of the latitudinal stellar rotation period $\left(P_{*}\right)$ and skyprojected orbital obliquity (the sky projection of the angle between the orbit of the planet and the spin of the host star), i.e. $\lambda$ from the transit light curves (e.g. Sanchis-Ojeda et al. 2011; Sanchis-Ojeda \& Winn 2011; Tregloan-Reed et al. 2013, 2015; Southworth et al. 2016; Mancini et al. 2017).

Over the years there have been multiple transit-starspot models developed both by the eclipsing binary star community (e.g. Wilson-Devinney code: Wilson \& Devinney 1971; Wilson 1979, 1990, 2008, 2012; PHEOBE: Prša \& Zwitter 2005; Prša et al. 2016) and the exoplanet community (e.g. PRISM: Tregloan-Reed et al. 2013, 2015, 2018; SOAP-T: Oshagh et al. 2013; spotrod: Béky et al. 2014; KSint: Montalto et al. 2014; ellc: Maxted 2016; StarSim: Herrero et al. 2016; PyTranSpot: Juvan et al. 2018). With a large variety of models readily available to the community, the treatment of starspot anomalies and their associated effects on light curves no longer cause difficulties or delays in the analysis of the exoplanetary light curves.

Locating starspot anomalies in light curves of smaller planets requires a significantly higher precision in the light curve because the size of the starspot anomaly scales linearly with the area of the planetary disc (Tregloan-Reed et al. 2013). This precludes the use of ground-based data for small planets, but the remarkable quality of the light curves from dedicated space missions such as the Kepler satellite (Borucki et al. 2010) makes this work viable. Whilst the temporal sampling of Kepler data (30 min cadence) is too low to resolve starspot anomalies for the vast majority of the stars observed, the NASA's TESS will observe $\geq 200000$ stars at a 2 min cadence (Stassun et al. 2018) and provide a large set of light curves suitable for detecting and measuring starspots.

The TESS instrument is spending 2018 and 2019 looking for super-Earth planets $\left(<2 R_{\oplus}\right)$ transiting between 200000 and 400000 pre-selected stars at a 2 min cadence (Stassun et al. 2018) with a particular emphasis on M dwarf stars. Recent simulations for TESS indicate the potential to detect 1700 planets from the smallest selection of pre-selected stars (Sullivan et al. $2015,2017)$. With 1100 of these planets predicted to be subNeptune ( $2 R_{\oplus}$ to $4 R_{\oplus}$ ) class planets, while, 419 super-Earth or smaller $\left(<2 R_{\oplus}\right)$ planets are expected to be found orbiting M dwarfs. With a further 137 super-Earth or smaller planets orbiting F, G, K stars (Sullivan et al. 2015, 2017). TESS is already exceeding early expectations with 21 confirmed planets (e.g. $\mathrm{Pi}$ Mensae c: Huang et al. 2018: LHS 3884 b; Vanderspek et al. 2019: HD 21749 b; Dragomir et al. 2019: HD 202772A b; Wang et al. 2019) with a further 336 TESS Objects of Interest (ToIs) ${ }^{1}$.

The objective of this work is to determine the detection limits of starspot anomalies in TESS exoplanetary transit light curves. This is accomplished by varying the variables that directly or indirectly alter the duration and/or amplitude of the starspot anomaly in the light curve and comparing the results against the photometric noise of the light curve: starspot size $\left(r_{\text {spot }}\right)$ and temperature $\left(T_{\text {spot }}\right)$, host star radius $\left(R_{*}\right)$ and effective temperature $\left(T_{\text {eff }}\right)$, planetary radius $\left(R_{\mathrm{p}}\right)$, semi-major axis $(a)$, orbital period $(P)$, orbital inclination $(i)$, observing cadence, and the frequency $(v)$ of the observation. Through this, it is possible to determine the physical properties of starspots at the detection limits for TESS transit light curves.

The outline of this paper is as follows. Section 2 describes how the contrast of the starspots was calculated in the TESS

1 From TESS data MAST page; accessed 2019-06-24. passband for use in the simulations and describes which parameters influence the size and shape of the starspot anomalies. The section then describes how the simulations were designed using the PRISM model to produce the simulated light curves. Section 3 presents the results, separated into three host star spectral types: K5V dwarf, M1V, and M4V dwarf stars. Section 4 discusses the results and gives the overall conclusions to the simulations.

\section{Simulating starspot anomalies with PRISM}

We used the transit-starspot model PRISM (Tregloan-Reed et al. $2013,2015,2018)$ to perform the simulations in this work. The PRISM $^{2}$ model is written in IDL $^{3}$ (Interactive Data Language) and uses a pixellation approach to model the stellar disc in a 2D array by subdividing the star into many individual elements. These elements can then be described by a $2 \mathrm{D}$ vector in Cartesian coordinates. Each element is then assigned an intensity value based on whether a stellar feature is present at that location and then the quadratic limb darkening law is applied over the entire stellar disc. The planet is then set to transit the star. For each data point in the transit light curve, the total received intensity is calculated based on which elements of the star are visible. The pixellation method is an ideal method to introduce stellar features to the stellar disc because the model allows individual intensities to be assigned to individual elements at specific coordinates (see Tregloan-Reed et al. 2013). This allows researchers to use PRISM to model both occulted and unocculted starspots and thus help "rule out" starspot trends when examining wavelength dependent transit depth (planetary radii) variations.

The original version of PRISM used six parameters to model the light curve: the ratio of the planetary to stellar radii $(k)$, the sum of the fractional $\left(r_{\mathrm{p}}=R_{\mathrm{p}} / a\right.$ and $\left.r_{*}=R_{*} / a\right)$ planetary and stellar radii $\left(r_{\mathrm{p}}+r_{*}\right)$, linear and quadratic coefficients of the quadratic limb darkening law $\left(u_{1}\right.$ and $\left.u_{2}\right)$, orbital inclination $(i)$, and time of minimum light $\left(T_{0}\right)$. These were combined with four additional parameters for each starspot: the longitude of the centre of the $\operatorname{spot}(\theta)$, the co-latitude of the centre of the $\operatorname{spot}(\phi)$, the angular size $\left(r_{\text {spot }}\right)$ and contrast $\left(\rho_{\text {spot }}\right)$ (TregloanReed et al. 2013). Since its publication, PRISM has had two major updates. The first added two orbital parameters: orbital eccentricity $(e)$ and argument of periastron $(\omega)$ (Tregloan-Reed et al. 2015). The second major update included a third light parameter and the out-of-transit detrending polynomial coefficients (Tregloan-Reed et al. 2018).

During its development the ability of PRISM to model the stellar disc and transit was tested via JKTEBOP (Southworth et al. 2004, 2005, 2007, 2009; Southworth 2008, 2010, 2011, 2013) as a benchmark transit modelling program. To accomplish this, a series of light curves were generated by both PRISM and JKTEBOP using the same transit parameter values. The average difference between the two models for all the tests was $\sim 10 \mathrm{ppm}$, which is six times lower than the expected noise floor in TESS data (see Sullivan et al. 2015).

Since the development of PRISM other researchers have used the model to ascertain the photometric parameters of a transiting system and to derive the parameters of the detected starspots observed in transit light curves (e.g. Mancini et al. 2013, 2014; Mohler-Fischer et al. 2013; Chen et al. 2017). The PRISM model has also been used to help calibrate and benchmark other

\footnotetext{
2 The latest version of PRISM is available from GitHub

3 For further details see http://www.harrisgeospatial.com/ ProductsandTechnology/Software/IDL . aspx
} 
transit-starspot models (e.g. spotrod: Béky et al. 2014; PyTranSpot: Juvan et al. 2018).

The PRISM model measures $r_{\text {spot }}$ in units of angular radius $\left(^{\circ}\right)$. Consequently, an $n^{\circ}$ starspot maintains a constant ratio of the stellar to starspot surfaces for all values of $R_{*}$. The simulations in this work use three different host stars, and as such, three different $R_{*}$. To avoid the projection effects on the spherical surface of the star we quote the starspot stellar surface radius in units of $R_{*}$, i.e.,

$n^{\circ} \equiv n^{\circ}\left(\frac{\pi}{180^{\circ}}\right) R_{*}$,

where a starspot with an angular radius of $1^{\circ}$ has a stellar surface radius of $0.017 R_{*}$

\subsection{Typical starspot contrast in the TESS passband}

While the temperature of the starspot $\left(T_{\text {spot }}\right)$ is fixed across all wavelengths, $\rho_{\text {spot }}$ is not, it is dependent on the frequency of the observation, $v$. At bluer wavelengths $\rho_{\text {spot }}$ decreases (higher contrast), increasing the amplitude of the starspot anomaly. This is because a starspot is cooler than the surrounding photosphere and both the starspot and photosphere can be treated as black bodies (Rabus et al. 2009; Sanchis-Ojeda \& Winn 2011). When the observation wavelength shifts towards a redder wavelength, $\rho_{\text {spot }}$ increases (lower contrast) and therefore the amplitude of the starspot anomaly can potentially fade into the observational noise.

Assuming that both the starspot and surrounding photosphere radiate as black bodies, Silva (2003) used the ratio of the intensities of the starspot to the surrounding photosphere to give an equation to find $\rho_{\text {spot }}$ from the stellar effective temperature $\left(T_{\text {eff }}\right), T_{\text {spot }}$ and $v$. By doing so, Silva (2003) incorporated the spectral signature of the starspot into $\rho_{\text {spot }}$,

$\rho_{\text {spot }}=\frac{\exp \left(h v / k_{\mathrm{B}} T_{\text {eff }}\right)-1}{\exp \left(h v / k_{\mathrm{B}} T_{\text {spot }}\right)-1}$,

where $h$ is Planck's constant and $k_{B}$ is Boltzmann constant.

The TESS instrument uses its own $T$ (TESS) passband with a spectral response between $600-1000 \mathrm{~nm}$ with the JohnsonCousins $I_{\mathrm{C}}$ passband lying near the centre of the $T$ passband (Sullivan et al. 2015). The effective wavelength midpoint of the $I_{\mathrm{C}}$ passband lies approximately at $785 \mathrm{~nm}$. We used Eq. (2) to calculate the contrast for a typical starspot on a cool M dwarf at 600,785 , and $1000 \mathrm{~nm}$ because a large proportion of the emitted flux from an $\mathrm{M}$ dwarf lies within the optical red infrared, potentially engulfing the starspot signal owing to the redder observational wavelengths. Using a $T_{\text {eff }}=3200 \mathrm{~K}$ (transit detection limit in $T$ passband; Sullivan et al. 2015) and setting $T_{\text {spot }}$ to be $200 \mathrm{~K}$ cooler than the photosphere (i.e. $T_{\text {spot }}=3000 \mathrm{~K}$ ), in line with starspot temperatures of fully convective $\mathbf{M}$ dwarf stars (e.g. Barnes et al. 2015), $\rho_{\text {spot }}$ was calculated to be $\rho_{\text {spot }}=0.60$, $\rho_{\text {spot }}=0.68$, and $\rho_{\text {spot }}=0.74$ for the three respective wavelengths. The amplitude of a starspot anomaly is primarily dependent on $r_{\text {spot }}$ and $\rho_{\text {spot }}$. However, there is a critical threshold for $\rho_{\text {spot }}$ at which the starspot anomaly is no longer detected, irrespective of $r_{\text {spot }}$. Previous works using ground-based telescopes have found starspot anomalies with $\rho_{\text {spot }}>0.74$ (e.g. HATP-36, $\rho_{\text {spot }}=0.92$ : Mancini et al. 2015; WASP-41, $\rho_{\text {spot }}=0.90$ : Southworth et al. 2016; WASP-6, $\rho_{\text {spot }}=0.80$ : Tregloan-Reed et al. 2015; WASP-19, $\rho_{\text {spot }}=0.78$ : Tregloan-Reed et al. 2013). This indicates that $\rho_{\text {spot }}$ of a typical starspot anomaly lying within the photosphere of a $\mathrm{M}$ dwarf star, observed in the $T$ passband, is below the critical threshold to be detected by ground-based telescopes and implies that starspot anomalies should be detected in TESS transit light curves.

\subsection{Parameter impact on the shape of a starspot anomaly}

Before we begin simulating starspot anomalies in transit light curves, we need to examine the parameters which directly or indirectly influence the size (amplitude) and shape (duration) of the anomaly. The parameters can be divided into four groups: starspot parameters, stellar and planetary parameters, orbital parameters, and observing constraints.

The first group of parameters belong to the starspot itself $\theta, \phi$, $r_{\text {spot }}$, and $\rho_{\text {spot }}$ (or $T_{\text {spot }}$ ). The amplitude of a starspot anomaly is directly proportional to $\rho_{\text {spot }}$ and the surface area of the starspot (Tregloan-Reed et al. 2013). As $\rho_{\text {spot }}$ decreases (i.e. the starspot darkens and cools), $r_{\text {spot }}$ must be reduced, to maintain the amplitude of the starspot anomaly. In essence, a small starspot needs a large ratio of $T_{\text {spot }}$ to $T_{\text {eff }}$ to be detected (see Eq. (2)). The location of the starspot, $\theta$ and $\phi$ have an indirect effect on the amplitude and duration of the anomaly. When the position of the starspot approaches the limb of the star, there is a reduction in the 2D projection of the surface area of the spot as a consequence of foreshortening along the radial vector, i.e. the vector between the centres of the stellar disc and starspot. Hence, this reduces the amplitude of the starspot anomaly. As the radial vector rotates (by altering the Azimuth angle) to being parallel with the transit cord, the duration of the anomaly is reduced too.

The next group of parameters are related to the host star; $T_{\mathrm{eff}}$, $R_{*}$, and the planet; $R_{\mathrm{p}}$. It was shown in both Sect. 2.1 and Eq. (2) how $T_{\text {eff }}$ directly alters $\rho_{\text {spot }}$ thereby indirectly influencing the amplitude of the anomaly. The amplitude of a starspot anomaly is also directly proportional to $k$, the ratio of $R_{\mathrm{p}}$ to $R_{*}$ (TregloanReed et al. 2013). If $k$ is too small in relation to the noise then the change in flux due to the planet occulting the starspot will not be detectable over the noise in the light curve.

The orbital parameters $i, a$, and $P$ all indirectly impact the amplitude and duration of the starspot anomaly. The parameter $i$ controls the impact parameter $(b)$ and therefore $i$ controls the stellar latitude at which the transit cord crosses. Because occulted starspots must lie on the transit chord to be observed then as $i$ decreases, the position of the starspot moves towards the stellar pole (i.e. the limb of the stellar disc). By doing so, the $2 \mathrm{D}$ projection of the surface area of the starspot decreases owing to foreshortening. The parameter $b$ is also controlled by $a$ combined with the orbital eccentricity $(e)$ and the argument of periastron $(\omega)$ (e.g. Winn 2010), i.e.

$b=\frac{a \cos i}{R_{*}}\left(\frac{1-e^{2}}{1+e \sin \omega}\right)$.

Because $P$ is related to $a$ via Kepler's third law and directly controls the transit duration (e.g. Winn 2010), it becomes apparent how $a$ and $P$ can both directly control the duration and indirectly influence the amplitude of the starspot anomaly.

The final group consists of just two parameters, $v$ and the observing cadence. In Sect. 2.1 it was shown how $v$ alters the amplitude of the starspot anomaly. Where the amplitude of the starspot anomaly increases towards bluer wavelengths and reduces towards redder wavelengths. The observing cadence however does not physically affect the size or shape of the starspot anomaly. However, it does alter our perception of the size and shape. When the cadence decreases (from high to low 
cadence), the number of data points that describe the starspot anomaly decreases, altering our perception of the true shape of the anomaly. This increases the uncertainty in the duration of the anomaly and thus increases the uncertainty in the measured parameters which influence the duration of the starspot anomaly.

Because these simulations are based on TESS transit light curves, the observational cadence is fixed at $2 \mathrm{~min}$ (Stassun et al. 2018) and $v$ is fixed at 600, 785, and $1000 \mathrm{~nm}$ (Sullivan et al. 2015). Consequently both the cadence and $v$ are considered as constraints and are used in the simulations at fixed values.

\subsection{Simulating starspots in TESS transit light curves}

The primary goal of this work is to find the detection limits of starspot anomalies appearing within TESS exoplanet light curves. The TESS instrument was developed to find small planets $\left(<6 R_{\oplus}\right)$ orbiting local $\mathrm{K}$ and $\mathrm{M}$ dwarf stars (Ricker et al. 2009). As a result of this, the host star and planetary properties used in these simulations were based on the selections used by Sullivan et al. (2015, 2017), covering K and M late-type stars with small planets, $<6 R_{\oplus}$.

When predicting the planetary yields of TESS Sullivan et al. (2015) calculated that TESS would have a photometric sensitivity of $200 \mathrm{ppm}$ for an $I_{\mathrm{C}} 10$ th magnitude star. Because of this, the light curves generated by PRISM had added Gaussian noise of $60 \pm 3^{4}, 100 \pm 5,150 \pm 7.5$, and $200 \pm 10 \mathrm{ppm}$. This approach allows us to simulate the photometric sensitivity of light curves for host stars brighter than $I_{C}=10$, after undergoing the TESS data processing pipeline and known systematics removal (see Jenkins et al. 2016).

With the TESS observations being performed in the $T$ passband (600-1000 nm; Sullivan et al. 2015) the simulations were run at three wavelengths: 600,785 , and $1000 \mathrm{~nm}$. These wavelengths were selected to give a good average approximation to the $T$ passband $(785 \mathrm{~nm})$ and to obtain results at the bluest $(600 \mathrm{~nm})$ and reddest $(1000 \mathrm{~nm})$ edge of the $T$-passband spectral response, thereby producing the largest and smallest amplitudes for the starspot anomalies.

Examining the range of parameters described in Sect. 2.2 it becomes apparent that the parameter space which controls the amplitude and duration of a starspot anomaly is large and complex due to multiple degeneracies between the parameters (e.g. $r_{\text {spot }} \leftrightarrow \rho_{\text {spot }}, \phi \leftrightarrow i, P \leftrightarrow a$, and $\phi \leftrightarrow P$ ). However, for this work we are only interested in the detection limits of starspot anomalies found within TESS light curves (i.e. smallest $r_{\text {spot }}$ for a given scenario). Therefore, we can streamline the parameter space by using a mixture of predetermined key parameter values and scenarios (e.g. setting $R_{*}$ and $T_{\text {eff }}$ for typical $\mathrm{K}$ and $\mathrm{M}$ dwarf stars) and search for the smallest detectable $r_{\text {spot }}$.

In the simulations the position of the starspot was set at the centre of the stellar disc, therefore minimising the effect from foreshortening. As discussed in Sect. 2.2 foreshortening reduces the amplitude of the starspot anomaly. Therefore, owing to the geometric nature of spherical foreshortening, the smallest detectable $r_{\text {spot }}$ is for starspots lying at the centre of the stellar disc. This is because when the starspot moves closer to the limb, $r_{\text {spot }}$ needs to increase so as to maintain the same 2D projected surface area of the starspot. Because we want to detect the smallest starspot for a given set of circumstances placing the starspot at the centre of the stellar disc achieves this result.

To determine TESS planetary yields Sullivan et al. (2015, 2017) used an M4V dwarf with $T_{\text {eff }}=3200 \mathrm{~K}$, a radius of

\footnotetext{
4 Mission specified noise floor at $60 \mathrm{ppm} \mathrm{hr}^{1 / 2}$ (see Sullivan et al. 2015).
}

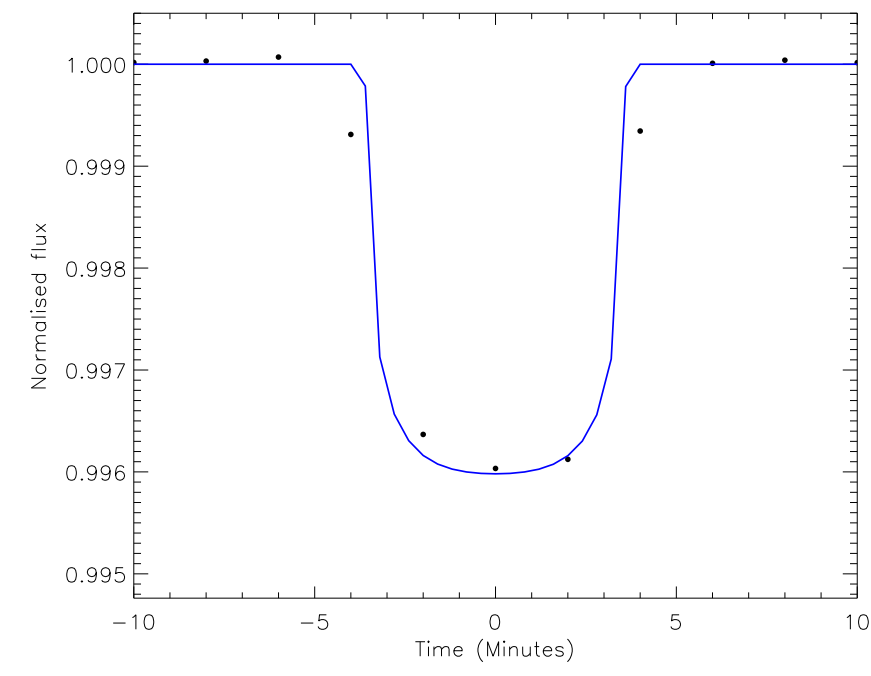

Fig. 1. Simulated 2 min cadence TESS light curve of a $1 R_{\oplus}$ planet orbiting an M4V dwarf star with $P=12 \mathrm{~d}$ and an rms scatter of $104 \mathrm{ppm}$ (generated by PRISM). The transit duration is approximately $8 \mathrm{~min}$ and only 3 data points lie within the transit.

$R_{*}=0.155 R_{\odot}$, a solar metallicity, and an age of 1 Gyr (in keeping with the Dartmouth Stellar Evolution Database; Dotter et al. 2008). The $T_{\text {eff }}$ was selected because $M$ dwarfs cooler than $3200 \mathrm{~K}$ are too faint for planetary transit detection in the $T$ passband (Sullivan et al. 2015). For this work it was therefore natural to start with an M4V dwarf star. The smaller value for $R_{*}$ and by association, larger $k$, meant that a $1 R_{\oplus}$ radius could be used in the M4V dwarf simulations. The planetary radii used ranged from 1 to $3 R_{\oplus}$ in $0.25 R_{\oplus}$ increments. An upper limit of $3 R_{\oplus}$ was used because of the rare occurrence of close in $(<50 \mathrm{~d})$, large $\left(>3 R_{\oplus}\right.$ ) planets orbiting $\mathrm{M}$ dwarf stars, determined from population studies using Kepler and K2 data (Dressing \& Charbonneau 2013, 2015; Morton \& Swift 2014). Although the recent discovery of NGTS-1 b, an $\approx 15 R_{\oplus}$, hot Jupiter transiting an M0.5 dwarf star with a $2.65 \mathrm{~d}$ orbital period (Bayliss et al. 2018) along with two previously known close-in large planets transiting $\mathbf{M}$ dwarfs (Kepler-45 b; Johnson et al. 2012: HATS-6 b; Hartman et al. 2015) appears to be lifting this limit.

A key objective of TESS is to find suitable planets for further study within the habitable zone of the host star. A planet orbiting an M4V dwarf star with a relative insolation $\left(S / S_{\oplus}\right)$ set at the inner $\left(S / S_{\oplus}=1.0\right)$, mean $\left(S / S_{\oplus}=0.6\right)$, and outer edge $\left(S / S_{\oplus}=0.2\right)$ of the habitable zone (Kopparapu et al. 2013) has an orbital period between 12 and 40 days (Eq. (13) Sullivan et al. 2015). The difficulty in detecting starspot anomalies at these orbital periods is that the transit duration, is less than $10 \mathrm{~min}$ (see Fig. 1). This results in the duration of a starspot anomaly being less than a minute, which is not favourable when using 2 min cadence data. Unlike transit detections, which can use phasefolded light curves to increase the number of data points within the transit and therefore aid in the transit detection, a starspot anomaly can generally only be detected using a single transit. This is the result of the rotation of the host star smearing the different positions of the starspot anomaly when viewing multiple phase-folded light curves. Because of this, the orbital period in the M4V dwarf simulations was set at 1,2, and 3 days to allow sufficient sampling in the single light curves to detect a starspot anomaly. It should be stated however that a subset of TESS data will be made available with a cadence of less than two minutes (20 s) for the asteroseismology community (Ricker et al. 2015). 
Table 1. Calculated values of $a$ for each value of $P$ for the three simulated host stars.

\begin{tabular}{cccccccc}
\hline \hline \multicolumn{2}{c}{ M4V } & & \multicolumn{2}{c}{ M1V } & & \multicolumn{2}{c}{ K5V } \\
\cline { 7 - 8 }$P(\mathrm{~d})$ & $a(\mathrm{AU})$ & & $P(\mathrm{~d})$ & $a(\mathrm{AU})$ & & $P(\mathrm{~d})$ & $a(\mathrm{AU})$ \\
\hline 1.0 & 0.0091 & & 2.0 & 0.0242 & & 2.0 & 0.0269 \\
2.0 & 0.0144 & & 3.0 & 0.0317 & & 4.0 & 0.0427 \\
3.0 & 0.0189 & & 4.0 & 0.0383 & & 6.0 & 0.0560 \\
\hline
\end{tabular}

Table 2. Extrapolated values of $R_{*}$ and $M_{*}$ using $T_{\text {eff }}$ from polynomials derived from interferometric measurements of $\mathrm{M}$ and $\mathrm{K}$ dwarf stars (Eqs. (8) and (10), Boyajian et al. 2012), for the three simulated host stars combined with the calculated $\log g$ and $u_{1}$ and $u_{2}$ for the quadratic limb darkening law from Claret (2017).

\begin{tabular}{lcccccc}
\hline \hline $\begin{array}{l}\text { Spectral } \\
\text { type }\end{array}$ & $\begin{array}{c}T_{\mathrm{eff}} \\
(\mathrm{K})\end{array}$ & $\begin{array}{c}R_{*} \\
\left(R_{\odot}\right)\end{array}$ & $\begin{array}{c}M_{*} \\
\left(M_{\odot}\right)\end{array}$ & $\begin{array}{c}\log g \\
(\mathrm{cgs})\end{array}$ & $u_{1}$ & $u_{2}$ \\
\hline M4V & 3200 & 0.155 & 0.10 & 5.06 & 0.1533 & 0.4776 \\
M1V & 3700 & 0.493 & 0.52 & 4.77 & 0.1737 & 0.4118 \\
K5V & 4100 & 0.623 & 0.65 & 4.66 & 0.3955 & 0.2618 \\
\hline
\end{tabular}

Kepler's third law was used to calculate $a$ from $P$ using a stellar mass, $M_{*}=0.1 M_{\odot}$ these are given in Table 1 . The stellar mass was determined using $R_{*}=0.155 R_{\odot}$ combined with Eq. (10) (second order polynomial) from Boyajian et al. (2012), which was derived from interferometric measurements of $\mathrm{M}$ and $\mathrm{K}$ dwarfs. We then calculated $\log g=5.06$ for the M4V dwarf and combined this value with $T_{\text {eff }}=3200 \mathrm{~K}$; we determined the quadratic limb darkening coefficients $u_{1}$ and $u_{2}$ (Table 2) with grids developed by Claret (2017) using stellar atmosphere models that are designed for use in the TESS passband.

Barnes et al. (2015) looked at starspot distributions of two fully convective M dwarfs, M4.5V: GJ 791.2 A, and M9V: LP 944-20 and found starspot temperatures around 200-300 K cooler than the surrounding photosphere. In line with this, we selected four equidistant starspot temperatures 3000, 3050, 3100, and $3150 \mathrm{~K}$ for the $3200 \mathrm{~K} \mathrm{M} 4 \mathrm{~V}$ dwarf simulations.

We used 1296 different scenarios for the M4V dwarf star simulations. Each scenario was derived from alternating the nine $R_{\mathrm{p}}$, three orbital periods, three observational wavelengths (600, 785 , and $1000 \mathrm{~nm}$ ), four starspot temperatures, and four noise levels $(60,100,150$, and $200 \mathrm{ppm})$. We then varied the $r_{\text {spot }}$ to find the detection limit for each of the 1296 scenarios. Figure 2 shows an example light curve and stellar disc from PRISM for one of the simulations.

Owing to the small radius $\left(R_{*}=0.155 R_{\odot}\right)$ of the initial host star, the second host star used in the simulations was a larger M1V dwarf star, where $T_{\text {eff }}=3700 \mathrm{~K}$ (Table 1 , Sullivan et al. 2015). This allowed the simulations to examine the detection limit of starspot anomalies at the two ends of the range of $R_{*}$ and $T_{\text {eff }}$ of $\mathrm{M}$ dwarfs observed by TESS. The $R_{*}$ and $M_{*}$ of the M1V dwarf were extrapolated using the polynomials derived from interferometric measurements of $\mathrm{M}$ and $\mathrm{K}$ dwarfs (Eqs. (8) and (10), Boyajian et al. 2012) using $T_{\text {eff }}=3700 \mathrm{~K}$. The results ${ }^{5}$ are given in Table 2 along with the extrapolated

5 We ignore the coefficient uncertainties in the polynomials presented by Boyajian et al. (2012) as we are generating hypothetical host stars, whose physical properties are consistent with the physical properties of observed stars of the same spectral type.
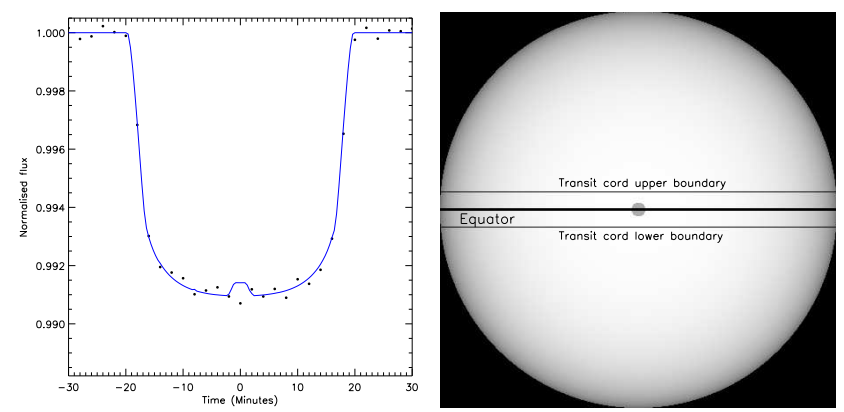

Fig. 2. Example light curve (left) and stellar disc (right) generated by PRISM. Light curve: the solid line represents the noise-free synthetic light curve containing the starspot anomaly, while, the filled circles represent the spot-free synthetic light curve (see Sect. 3) with added Gaussian noise. Stellar disc: the central solid line represents the stellar equator (assuming orbital alignment) and the upper and lower solid lines represent the upper and lower boundaries of the transit cord. These were generated with a $1.5 R_{\oplus}$ super-Earth planet transiting a $3200 \mathrm{~K}, 0.155 R_{\odot}$ M4V dwarf star with $i=90.0^{\circ}$ and $P=1 \mathrm{~d}$. The starspot properties are: $\theta=0^{\circ}, \phi=90^{\circ}, r_{\text {spot }}=0.035 R_{*}$ and $T_{\text {spot }}=3000 \mathrm{~K}$. The observational wavelength and rms scatter for the simulated data were set at $785 \mathrm{~nm}$ and $150 \pm 7.5 \mathrm{ppm}$, respectively.

properties of the M4V and $\mathrm{K} 5 \mathrm{~V}$ dwarf host stars. We employed the same procedure that was used for the M4V dwarf in determining $u_{1}$ and $u_{2}$ from Claret (2017) with the results reported in Table 2.

As a consequence of a threefold increase in the stellar radius, the planetary radii used in the M1V simulations ranged from 2 to $4 R_{\oplus}$ in $0.25 R_{\oplus}$ increments. Three orbital periods were selected at 2,3, and 4 days and the corresponding values for $a$ were calculated using Kepler's third law using a stellar mass of $0.52 M_{\odot}$ (see Table 2) and are given in Table 1 . Adopting $T_{\text {eff }}=3700 \mathrm{~K}$ for the M1V dwarf star, we selected four equidistant starspot temperatures, $3400,3475,3550$, and $3625 \mathrm{~K}$, giving a further 1296 different scenarios using an M1V dwarf host star; we used the same three observational wavelengths and four noise levels that were used in the M4V dwarf simulations. The same procedure that was used in the M4V simulations was used for the M1V simulations in that for each of the 1296 scenarios, $r_{\text {spot }}$ was varied to find the detection limit in each scenario.

For the third host star, a K5V dwarf star was selected. Using an $T_{\text {eff }}=4100 \mathrm{~K}$ (Table 1, Sullivan et al. 2015), the $R_{*}$ and $M_{*}$ was extrapolated from the two polynomials presented by Boyajian et al. (2012); these values are given in Table 2. We determined $u_{1}$ and $u_{2}$ from Claret (2017), for the K5V dwarf; these values are given in Table 2 .

Because of the larger $R_{*}$ the $\mathrm{K} 5 \mathrm{~V}$ simulations probed the detection limits of starspot anomalies for larger planets, 3-7 $R_{\oplus}$ (sub-Neptune to sub-Saturn class planets) in $0.5 R_{\oplus}$ increments. Owing to the larger $R_{*}$ and $R_{\mathrm{p}}$ the three values of $P$ were selected at 2, 4, and 6 days with the corresponding values for $a$ calculated using Kepler's third law with a stellar mass of $0.65 M_{\odot}$ (see Table 2); these values are given in Table 1 . With $T_{\text {eff }}=4100 \mathrm{~K}$ four temperatures were selected for the starspot 3700, 3800, 3900 , and $4000 \mathrm{~K}$. Combining the three observational wavelengths and four noise levels meant that a further 1296 scenarios were generated using a K5V dwarf host star.

\section{Simulation results}

For all 3888 different scenarios a total of 20573 simulated transits were generated. All 20573 simulated transit light curves are 
available at the CDS. The number of simulated transits per scenario was dependent on the detection of $r_{\text {spot }}$ and ranged from 2 to 40 simulations per scenario, where $r_{\text {spot }}$ started at $0.0087 R_{*}$ and was increased in $0.0087 R_{*}$ steps up to $0.35 R_{*}$. The simulations were stopped when the starspot anomaly was deemed detectable. The starspot anomaly was considered "detected" in the simulated transits, if the characteristic blip was visible in the simulated light curve (e.g. Fig. 2). This was accomplished by comparing the amplitude of the anomaly to the photometric precision (rms scatter) of the data. The amplitude of the starspot anomaly was measured by creating two transit light curves. The first had the centre of the starspot within the transit cord while the second light curve had the starspot latitude shifted so that it was no longer within the transit cord. Because both occulted and non-occulted starspots affect the shape of the light curve (Silva-Válio 2010) and in particular the transit depth (Nikolov et al. 2013) it was important that the stellar disc of the "spot-free" light curve model still contained a starspot of equal properties. To reduce the impact from foreshortening, the starspot was positioned so that only a gap of one pixel separated the starspot and transit cord boundaries. We compared the resulting light curves to determine the amount of systematic error the approximation would generate and found a mean divergence of $\approx 1 \mathrm{ppm}$, which is 60 times smaller than the TESS mission specified noise floor. Therefore, we conclude that this approximation is suitable for this work.

The light curve plots produced in this work show two synthetic light curves represented by a solid line and filled circles. The light curves represented by a solid line are the synthetic light curves containing the starspot anomaly and these do not have added noise. The light curves represented by filled circles are the synthetic light curves of the spot-free model and have added Gaussian noise. By comparing the two synthetic light curves it is possible to determine if the amplitude of the starspot anomaly is larger than the rms scatter.

To determine the most efficient set of detection conditions, tests were performed on the synthetic data. After the addition of noise to the synthetic light curve, PRISM was fitted to the synthetic light curve in an attempt to recover the starspot parameters. The amplitude of the starspot anomaly was determined by the data points which described the starspot anomaly and the mean change in flux was calculated. The mean change in flux was then divided by the rms scatter of the light curve to give the amplitude of the starspot anomaly in units of rms $(\sigma)$. Different amplitudes were considered from $1 \sigma$ to $3 \sigma$. In all cases where the amplitude was greater than $2.0 \sigma$, the best fitting starspot parameters of the synthetic light curves were close to the original values and agreed within their $1 \sigma$ parameter uncertainties. However, when the amplitude of the starspot anomaly was between $1.5 \sigma$ and $2 \sigma$, the $1 \sigma$ uncertainty in the starspot parameters was non-physical. For example in one test the starspot radius was found to be $0.047 R_{*} \pm 1.700 R_{*}$, indicating that the starspot covered the entire visible hemisphere of the stellar disc, and in another test the latitude of the starspot was found to be $0.17^{\circ} \pm 97.39^{\circ}$; this indicated that a spot-free solution, i.e. the starspot was no longer on the transit cord, was an acceptable solution. These tests showed that when the amplitude of the starspot anomaly was between $1.5 \sigma$ and $2 \sigma$ the anomaly could be detected but it was not possible to constrain the starspot parameters, thereby confirming the presence of a starspot. Therefore, the optimal cutoff point for the amplitude of the starspot anomaly was set at the $2 \sigma$ limit to differentiate between a detection and non-detection.

\subsection{M4V dwarf host star results}

A total of 6870 simulations were conducted for the 1296 scenarios using an M4V dwarf host star; the results are presented in Table A.1. It was found that the starspot detection limit in TESS transit light curves of an M4V dwarf host star was $r_{\text {spot }}=0.045 R_{*} \pm 0.016 R_{*}(4900 \pm 1700 \mathrm{~km})$, where the uncertainty is the $1 \sigma$ standard deviation from the 1296 scenarios of the M4V host star simulations. The smallest $r_{\text {spot }}$ detected in the M4V simulations (under optimal parameter conditions) was $r_{\text {spot }}=0.017 R_{*}$ or $1900 \mathrm{~km}$ (see Fig. 3) and was detected in 7 of the 1296 scenarios $(0.5 \%)$.

It was found that for three scenarios, it was not possible to resolve the starspot size (see Fig. 4). These scenarios involved a $3150 \mathrm{~K}$ starspot with a photometric precision of $200 \mathrm{ppm}$ observed at $1000 \mathrm{~nm}$ with a $1 R_{\oplus}$ transiting planet. When examining the stellar disc output for the $0.093 R_{*}$ starspot in Fig. 4, the area of the $2 \mathrm{D}$ projection of the starspot is larger than the planetary disc. Therefore, increasing $r_{\text {spot }}$ further, would no longer increase the amplitude of the starspot anomaly because it would not affect the amount of flux blocked by the planet. However, it increases the duration because the planet spends longer occulting the starspot. This can be seen when examining the three simulated light curves in Fig. 4. Therefore, for these scenarios, the amplitude of the starspot anomaly never became larger than the rms scatter of the synthetic data.

Hence, the simulations show that it is not possible to observe a $3150 \mathrm{~K}$ starspot on the surface of a $3200 \mathrm{~K}$ M4V dwarf, using a $1.0 R_{\oplus}$ Earth-sized planet, when observed at the reddest edge of the $T$ passband (i.e. $1000 \mathrm{~nm}$ ) with an rms scatter of $200 \mathrm{ppm}$. However, by either increasing the planetary radius $\left(+0.25 R_{\oplus}\right)$, reducing the rms scatter $(-50 \mathrm{ppm})$, or shifting to a bluer wavelength, it would be possible to detect these starspots down to $0.035 R_{*}(3800 \mathrm{~km})($ see Table A.1).

\subsection{M1V dwarf host star results}

A total of 7769 simulations were conducted for the 1296 scenarios using an M1V dwarf host star; these results are presented in Table A.2. It was found that the starspot detection limit in TESS transit light curves of an M1V dwarf host star was $r_{\text {spot }}=0.040 R_{*} \pm 0.017 R_{*}(13800 \pm 6000 \mathrm{~km})$. The smallest $r_{\text {spot }}$ detected in the M1V simulations (under optimal parameter conditions) was $r_{\text {spot }}=0.017 R_{*}$ or $6000 \mathrm{~km}$ (see Fig. 5), which was detected in 85 of the 1296 scenarios (6.6\%).

To allow for a direct comparison between the starspots on the M1V and M4V dwarf stars requires the M1V dwarf star, $r_{\text {spot }}$ to be multiplied by $\left(\frac{R_{\mathrm{M} 1 \mathrm{~V}}}{R_{\mathrm{M} 4 \mathrm{~V}}}\right) \approx 3.2$. From this it can be seen that while the smallest starspot detected in the M1V dwarf simulations had $r_{\text {spot }}=0.017 R_{*}$, this is the equivalent to $r_{\text {spot }} \approx 0.056 R_{*}$ for a M4V dwarf star.

Similar to the M4V host star results, the M1V host star results show that it is not possible to detect the hottest starspots when small planets (e.g. $<2.75 R_{\oplus}$ ) are transiting the star if the photometric precision is $>100 \mathrm{ppm}$.

\subsection{K5V dwarf host star results}

A total of 5934 simulations were conducted for the 1296 scenarios using a K5V dwarf host star; these results are presented in Table A.3. It was found that the starspot detection limit in TESS transit light curves of a K5V dwarf host star was $r_{\text {spot }}=0.038 R_{*} \pm 0.016 R_{*}(15900 \pm 6800 \mathrm{~km})$. The smallest $r_{\text {spot }}$ 
J. Tregloan-Reed and E. Unda-Sanzana: Simulations of starspot anomalies within TESS
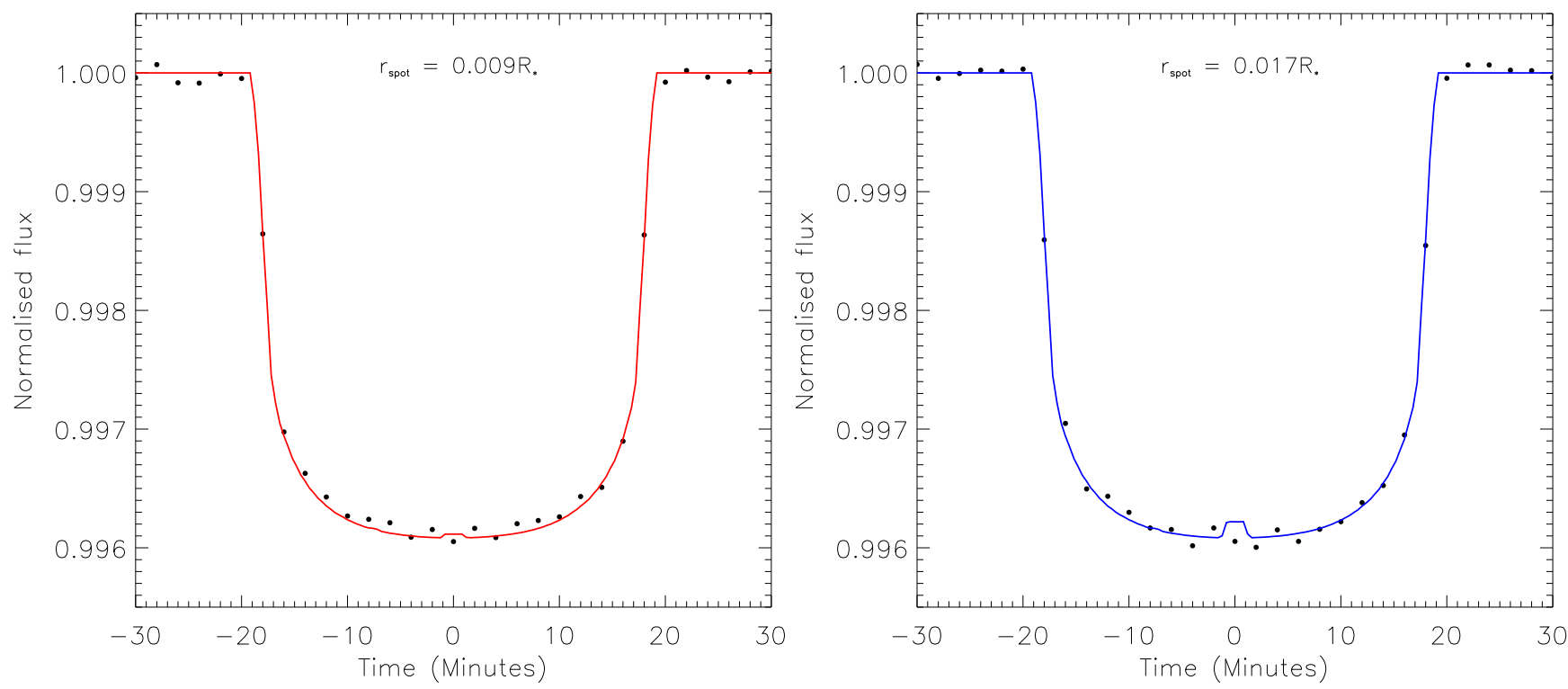

Fig. 3. Two simulated transit light curves generated by PRISM. These were both generated using a $1.0 R_{\oplus}$ Earth-sized planet transiting a $3200 \mathrm{~K}$, $0.155 R_{\odot} \mathrm{M} 4 \mathrm{~V}$ dwarf star with $i=90.0^{\circ}$ and $P=1 \mathrm{~d}$. The starspot properties are $\theta=0^{\circ}, \phi=90^{\circ}, r_{\text {spot }}=0.009 R_{*}(l e f t), r_{\text {spot }}=0.017 R_{*}($ right $)$, and $T_{\text {spot }}=3000 \mathrm{~K}$. The observational wavelength and rms scatter for the simulated transits were set at $600 \mathrm{~nm}$ and $60 \mathrm{ppm}$, respectively. The starspot anomaly becomes visible over the simulated noise when $r_{\text {spot }}$ is increased from $0.009 R_{*}$ to $0.017 R_{*}$. The solid lines represents the noise-free synthetic light curves containing the starspot anomaly, while the filled circles represent the spot-free synthetic light curves (see Sect. 3) with added Gaussian noise.
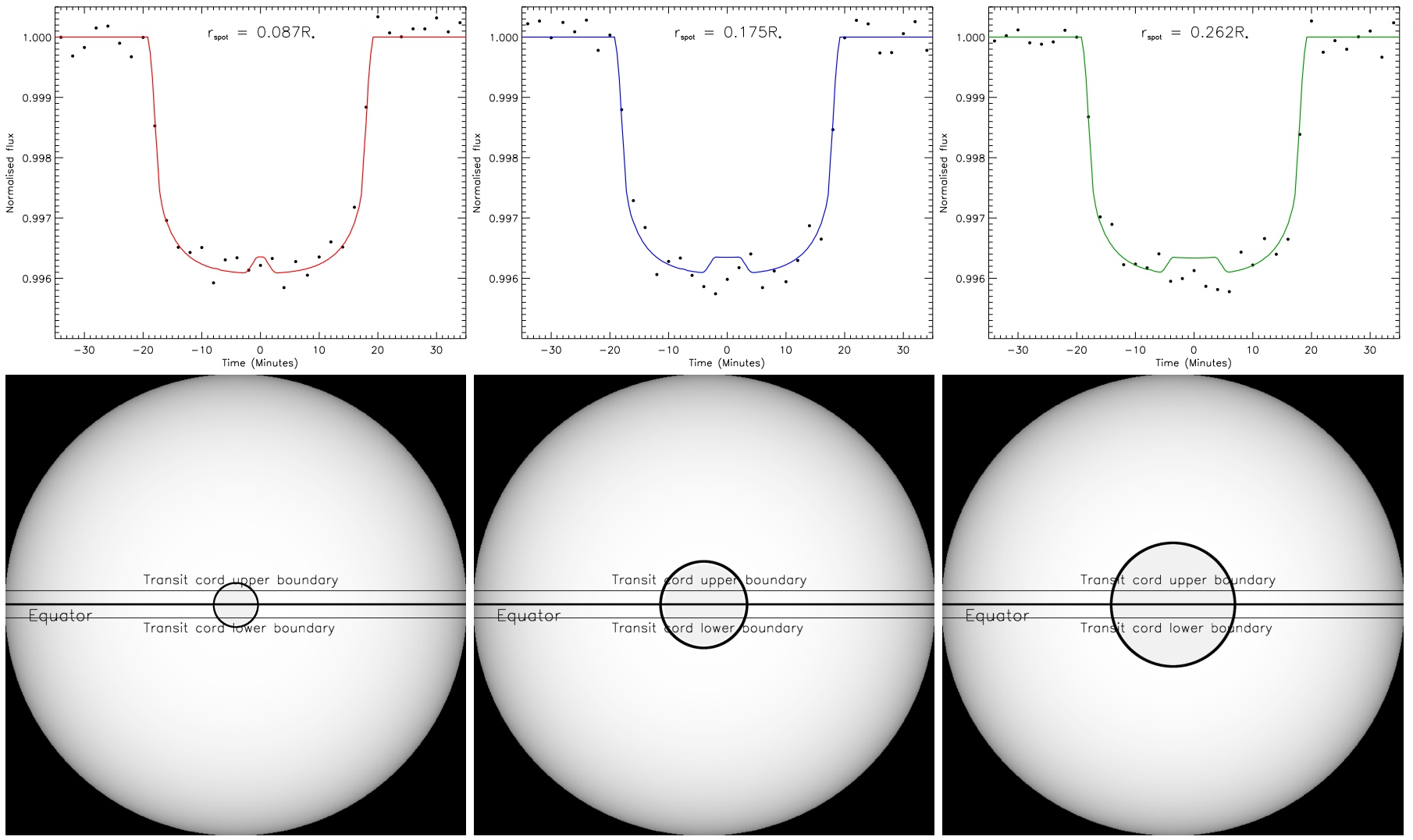

Fig. 4. Three simulated transit light curves (top panels) and stellar disc outputs (bottom panels), generated by PRISM. Light curves: the solid lines represent the noise-free synthetic light curves containing the starspot anomaly, while, the filled circles represent the spot-free synthetic light curves (see Sect. 3) with added Gaussian noise. Stellar discs: the central solid lines represents the stellar equator (assuming orbital alignment) and the upper and lower solid lines represent the upper and lower boundaries of the transit cords. Because the high $\rho_{\text {spot }}$, the starspot boundary is highlighted with a black circular boarder. The observational wavelength and rms scatter for the simulated transits are set at $1000 \mathrm{~nm}$ and $200 \mathrm{ppm}$, respectively. These were generated using a $1.0 R_{\oplus}$ Earth-sized planet transiting a $3200 \mathrm{~K}, 0.155 R_{\odot} \mathrm{M} 4 \mathrm{~V}$ dwarf star with $i=90.0^{\circ}$ and $P=1 \mathrm{~d}$. The starspot properties were set at $\theta=0^{\circ}, \phi=90^{\circ}$ and $T_{\text {spot }}=3150 \mathrm{~K}\left(\rho_{\text {spot }}=0.93\right)$. Left panels: $r_{\text {spot }}=0.087 R_{*}$, middle panels: $r_{\text {spot }}=0.175 R_{*}$, right panels: $r_{\mathrm{spot}}=0.262 R_{*}$. 

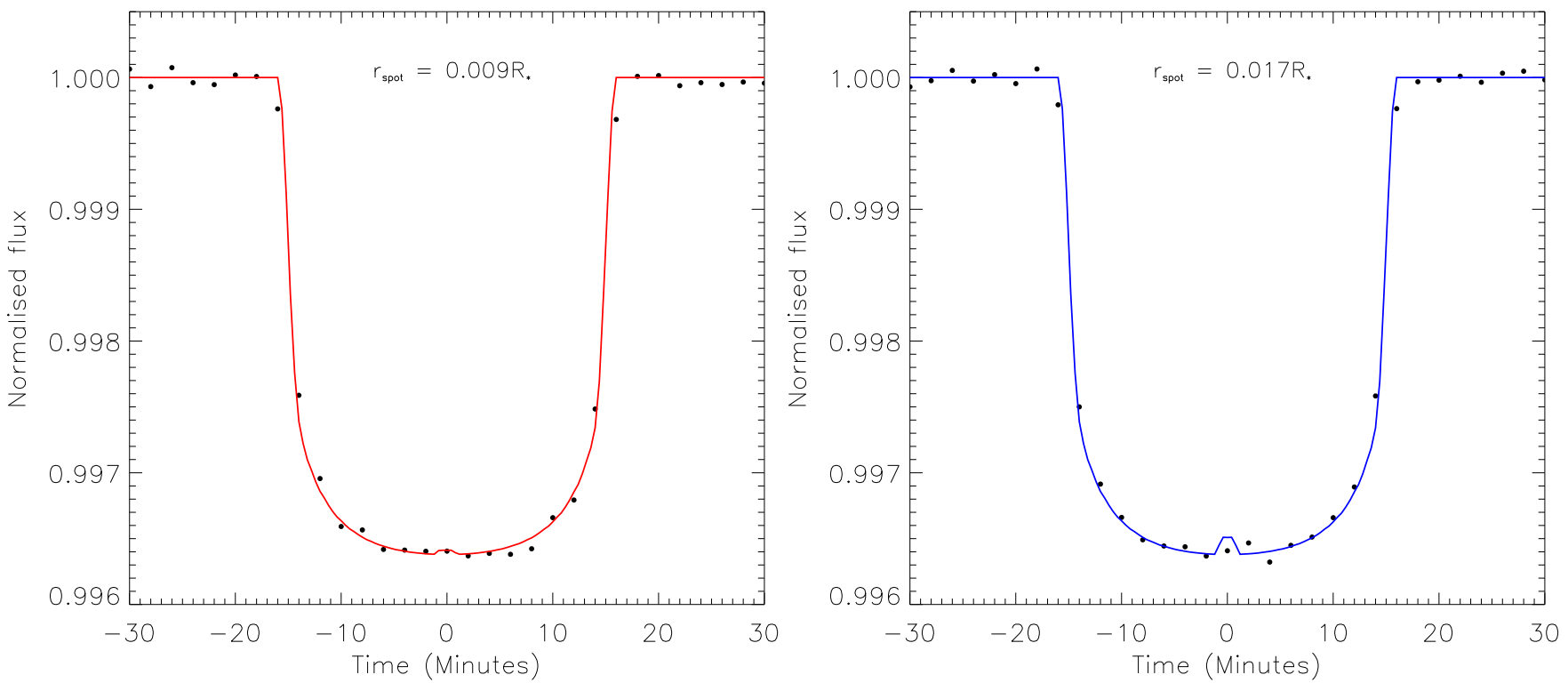

Fig. 5. Two simulated transit light curves generated by PRISM. These were both generated using a $2.75 R_{\oplus}$ sub-Neptune planet transiting a $3700 \mathrm{~K}$, $0.493 R_{\odot} \mathrm{M} 1 \mathrm{~V}$ dwarf star with $i=90.0^{\circ}$ and $P=3 \mathrm{~d}$. The starspot properties are $\theta=0^{\circ}, \phi=90^{\circ}, r_{\text {spot }}=0.009 R_{*}(l e f t), r_{\text {spot }}=0.017 R_{*}(r i g h t)$, and $T_{\text {spot }}=3400 \mathrm{~K}$. The observational wavelength and rms scatter for the simulated transits were set at $785 \mathrm{~nm}$ and $60 \mathrm{ppm}$, respectively. The starspot anomaly becomes visible over the simulated noise when $r_{\text {spot }}$ is increased from $0.009 R_{*}$ to $0.017 R_{*}$. The solid lines represent the noise-free synthetic light curves containing the starspot anomaly, while the filled circles represent the spot-free synthetic light curves (see Sect. 3) with added Gaussian noise.
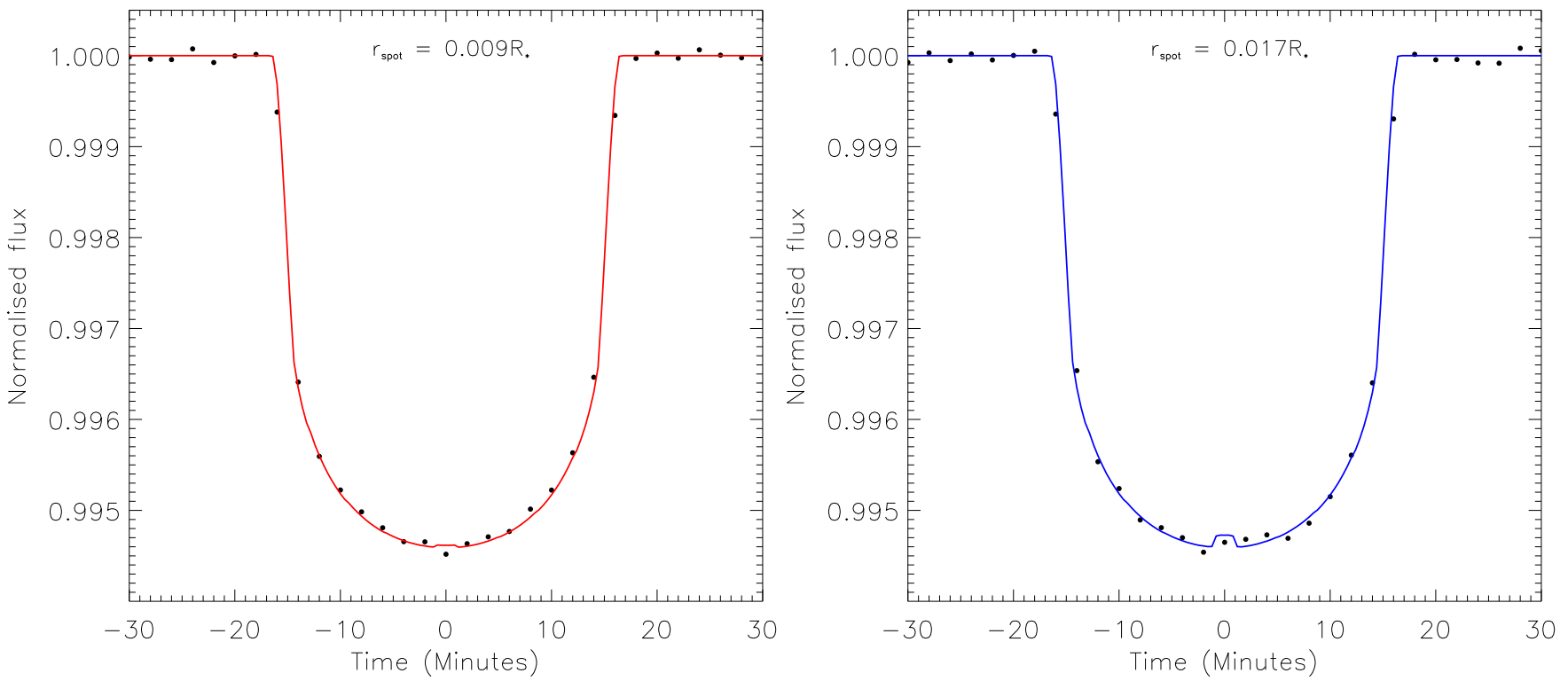

Fig. 6. Two simulated transit light curves generated by PRISM. These were both generated using a $4.5 R_{\oplus}$ Neptune-sized planet transiting a $4100 \mathrm{~K}$, $0.623 R_{\odot} \mathrm{K} 5 \mathrm{~V}$ dwarf star with $i=90.0^{\circ}$ and $P=4 \mathrm{~d}$. The starspot properties are $\theta=0^{\circ}, \phi=90^{\circ}, r_{\text {spot }}=0.009 R_{*}$ (red), $r_{\text {spot }}=0.017 R_{*}$ (blue), and $T_{\text {spot }}=3700 \mathrm{~K}$. The observational wavelength and rms scatter for the simulated transits were set at $785 \mathrm{~nm}$ and $60 \mathrm{ppm}$, respectively. The starspot anomaly becomes visible over the simulated noise when $r_{\text {spot }}$ is increased from $0.009 R_{*}$ to $0.017 R_{*}$. The solid lines represents the noise-free synthetic light curves containing the starspot anomaly, while the filled circles represent the spot-free synthetic light curves (see Sect. 3) with added Gaussian noise.

detected in the $\mathrm{K} 5 \mathrm{~V}$ simulations (under optimal parameter conditions) was $r_{\text {spot }}=0.017 R_{*}$ or $7600 \mathrm{~km}$ (see Fig. 6) and was detected in 102 of the 1296 scenarios (7.9\%).

We used the same process described in Sect. 3.2 to compare the starspot sizes on the $\mathrm{K} 5 \mathrm{~V}$ and M4V dwarf stars. We find that for the $\mathrm{K} 5 \mathrm{~V}$ dwarf star, $r_{\text {spot }}$ should be multiplied by $\left(\frac{0.623}{0.155}\right) \approx 4.0$. The smallest starspot detections for the K5V host star simulations were when $r_{\text {spot }}=0.017 R_{*}$, which would equate to a detection with $r_{\text {spot }} \approx 0.07 R_{*}$ if the starspots were on a M4V dwarf instead.

It was found that it was not possible to resolve the starspot for only $15(1.2 \%)$ scenarios. These scenarios followed the same pattern as found with the previous host stars. The scenarios involving a high temperature starspot, small planet, and that were observed at redder wavelengths with low photometric precision tended to prevent the detection of the starspot. 

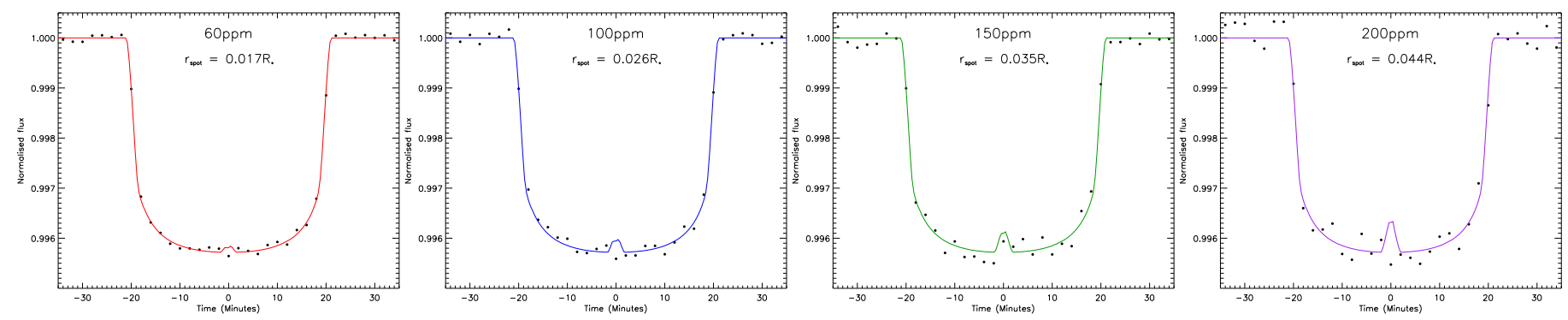

Fig. 7. Four simulated transit light curves generated by PRISM. The observational wavelength of the simulated transits was $785 \mathrm{~nm}$. These were generated using a $3.0 R_{\oplus}$ sub-Neptune planet transiting a $3700 \mathrm{~K}, 0.493 R_{\odot} \mathrm{M} 1 \mathrm{~V}$ dwarf star with $i=90.0^{\circ}$ and $P=2 \mathrm{~d}$. The starspot properties were set at $\theta=0^{\circ}, \phi=90^{\circ}$ and $T_{\text {spot }}=3475 \mathrm{~K}$. The rms scatter of the light curves from left to right are $60,100,150$, and $200 \mathrm{ppm}$. Each light curve shows the smallest detectable starspot size for the scenarios, $0.017 R_{*}$ (red), $0.026 R_{*}$ (blue), $0.035 R_{*}$ (green), and $0.044 R_{*}$ (purple), which increases with increasing noise. The solid lines represents the noise-free synthetic light curves containing the starspot anomaly, while the filled circles represent the spot-free synthetic light curves (see Sect. 3) with added Gaussian noise.

\subsection{Expected trends}

By examining the results from the 20573 simulated transits (see Tables A.1-A.3), known trends (described in Sect. 2.2) in the starspot detection limits are seen.

The simulations confirmed that increasing the photometric precision (decrease in noise) in the observations allowed smaller starspots to be detected (e.g. Fig. 7). The simulations also confirm that the starspot detection limit is dependent on $T_{\text {spot }}$, observational wavelength, and orbital period (e.g. Fig. 8). It is easier to detect a small cool starspot than a small hot starspot (top row, Fig. 8). Likewise, a shorter orbital period allows the detection of smaller starspots (bottom row, Fig. 8). This is because of a longer transit duration and, by association, a longer starspot anomaly duration, thereby increasing the number of data points describing both the transit and starspot anomaly becuase of fixed 2 min cadence of TESS data.

The simulations help support the predicted and known trends given in Sect. 2.2 and highlights which parameters the detection limit is strongly or weakly dependent on. Examining Fig. 8, we see that by doubling the orbital period $(P)$, the minimum detected $r_{\text {spot }}$ increases by $20 \%$, while shifting the observed wavelength from 600 to $1000 \mathrm{~nm}$, the minimum detected $r_{\text {spot }}$ increases by $50 \%$. However increasing $T_{\text {spot }}$ by $100 \mathrm{~K}$ increases the minimum detected $r_{\text {spot }}$ by $67 \% \%^{6}$, indicating that the detection limit is primarily influenced by $T_{\text {spot }}$ and $\lambda_{\mathrm{obs}}$; this is expected because the amplitude of a starspot anomaly is directly proportional to $\rho_{\text {spot }}$ (see Tregloan-Reed et al. 2013), which in turn is proportional to $T_{\text {spot }}$ and $\lambda_{\text {obs }}$ (see Eq. (2)).

The amplitude of the starspot anomaly is proportional to $\left(\frac{r_{\text {spot }}}{R_{\mathrm{p}}}\right)^{2}$ when $r_{\text {spot }} \leq R_{\mathrm{p}}$. Therefore, smaller planets can detect smaller starspots. The results from the simulations agree with this when dealing with cool starspots. However, the simulations depict a different trend when dealing with the hotter starspots in that larger planets are able to detect smaller starspots. For example in Table A.1 we can see that a $1.25 R_{\oplus}$ planet transiting a $3200 \mathrm{~K}, 0.155 R_{\odot} \mathrm{M} 4 \mathrm{~V}$ dwarf star with $i=90.0^{\circ}, P=2 \mathrm{~d}$, $\lambda_{\text {obs }}=785 \mathrm{~nm}$, and $T_{\text {spot }}=3150 \mathrm{~K}$, where the rms scatter is $150 \pm$ $7.5 \mathrm{ppm}$, the smallest detected $r_{\text {spot }}$ is $r_{\text {spot }}=0.070 R_{*}$ While for a $1.5 R_{\oplus}$ and $1.75 R_{\oplus}$ planet the smallest detected $r_{\text {spot }}$ becomes $r_{\text {spot }}=0.061 R_{*}$ and $r_{\text {spot }}=0.052 R_{*}$, respectively.

\footnotetext{
6 For a $3200 \mathrm{~K}$ M4V dwarf star observed at $600 \mathrm{~nm}$
}

\subsection{Unexpected trend}

Table 3 shows the comparison between $R_{\mathrm{p}}$ and the statistical mean of the starspot detection limit $\left(\bar{r}_{\text {spot }}\right)$, including its $1 \sigma$ uncertainty. At first glance, Table 3 seems to be in contradiction with the statement small planets are needed to detect small starspots. However, looking at the top row of Table 3, we see that when the $1 \sigma$ uncertainty in $\bar{r}_{\text {spot }}$ is taken into account, we see that the smallest planet (e.g. $R_{\mathrm{p}}=1 R_{\oplus}$ ) can detect the smallest starspot (e.g. $\bar{r}_{\text {spot }}=0.018 R_{*}$ ). The results in Table 3 also indicate that the range of values for $\bar{r}_{\text {spot }}$ decreases with increasing $R_{\mathrm{p}}$.

When examining Tables A.1-A.3, we can see an unexpected trend emerging: starspots that are small and hot can be detected by large planets, however, they cannot be detected by smaller planets. This unexpected and counter-intuitive trend, however, is a manifestation, from using a fixed cadence combined with the methodology used to detect starspot anomalies in transit light curves.

Increasing $R_{\mathrm{p}}$ not only increases the transit duration, it also increases the duration of the starspot anomaly. This is because a larger planetary disc spends longer occulting the starspot. For a fixed cadence ( $2 \mathrm{~min}$ for TESS) this equates to an increase in the number of data points which describe the starspot anomaly. As mentioned in Sect. 3 the mean flux of the data points within the starspot anomaly was used to determine if the starspot had been detected. This is in principle the same method used to detect starspot anomalies in observed light curves. In the case when a single data point describes the apex of a starspot anomaly (i.e. the 2 D projection of $r_{\text {spot }}$ equals $R_{\mathrm{p}}$; Fig. 9) the data points which lie within the ingress and egress of the starspot anomaly reduce the mean flux. For a starspot anomaly that is described by multiple data points which form a plateau - i.e. the $2 \mathrm{D}$ projection of $r_{\text {spot }}$ is either larger or smaller than $R_{\mathrm{p}}$ (Fig. 10) - the mean flux increases because the data points lying on the starspot ingress and egress have a weaker impact. Because of this, a starspot with an apex requires a larger amplitude to be detected. This is correct as a single data point should require a larger amplitude (compared to multiple data points) to allow a confirmed starspot detection. However, this creates an unavoidable detection bias.

Figures 9 and 10 depict two identical scenarios except that they have two different $R_{\mathrm{p}}, R_{\mathrm{p}}=1.0 R_{\oplus}$ (Fig. 9) and $R_{\mathrm{p}}=$ $1.75 R_{\oplus}$ (Fig. 10). The starspot $\left(r_{\text {spot }}=0.061 R_{*}\right)$ is not detected in Fig. 9, however by increasing the duration of the starspot anomaly and therefore the number of data points which describe this anomaly, the starspot is then detected (Fig. 10). 

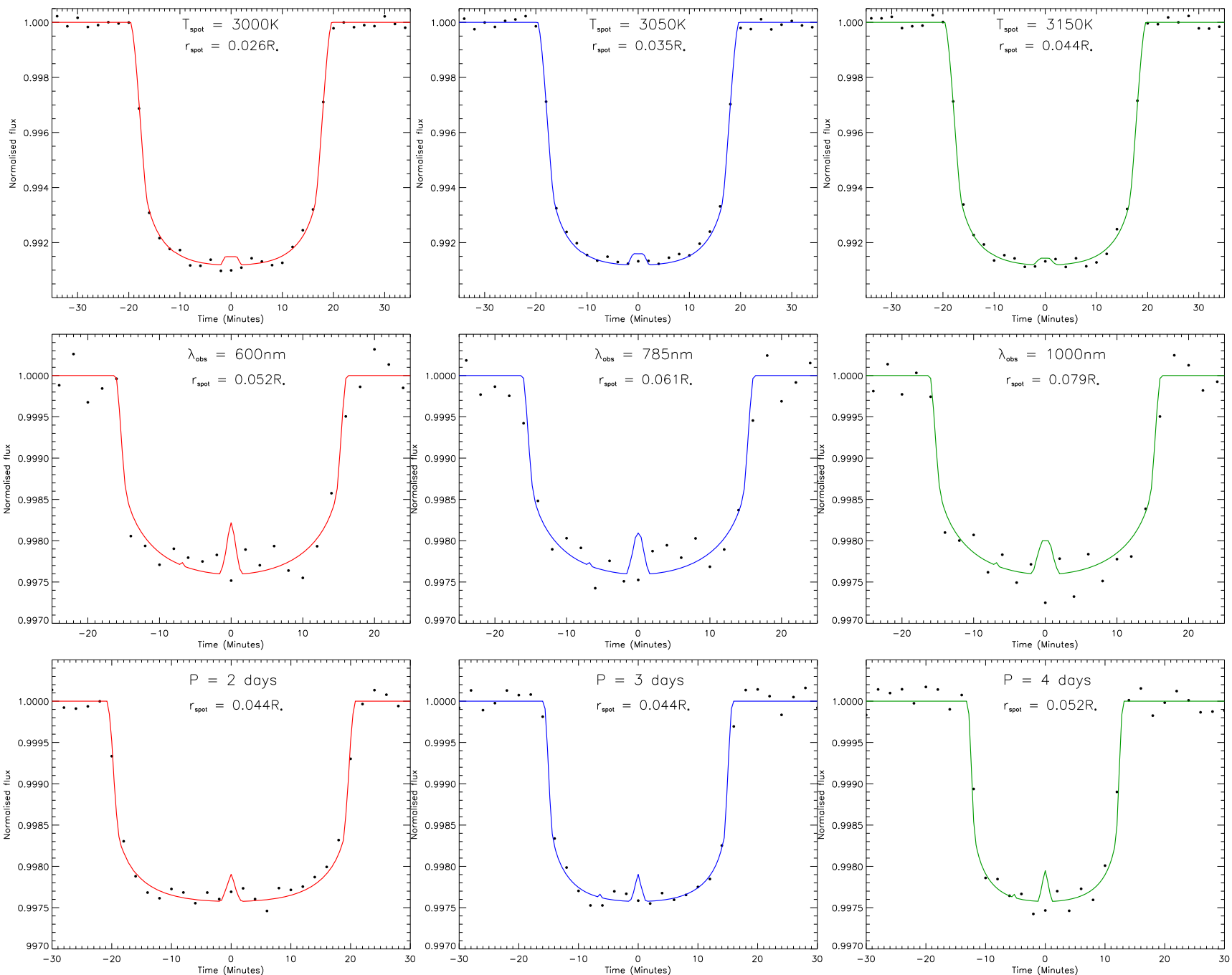

Fig. 8. Nine simulated transit light curves generated by PRISM, showing the smallest detected starspot for 3 trends, $T_{\text {eff }}:$ top row; $\lambda_{\text {obs }}:$ middle row; $P$ : bottom row. Top row: simulations of a $1.5 R_{\oplus}$ super-Earth planet transiting a $3200 \mathrm{~K}, 0.155 R_{\odot} \mathrm{M} 4 \mathrm{~V}$ dwarf star with $i=90.0^{\circ}, P=1 \mathrm{~d}$, $\lambda_{\text {obs }}=600 \mathrm{~nm}$, and rms scatter $150 \pm 7.5 \mathrm{ppm}$. Each column on the top row shows a different $T_{\text {spot }}$, where $T_{\text {spot }}=3000,3050$, and $3100 \mathrm{~K}($ left to right). The smallest detected $r_{\text {spot }}$ are $r_{\text {spot }}=0.026 R_{*}(\mathrm{red}), r_{\text {spot }}=0.035 R_{*}$ (blue), and $r_{\text {spot }}=0.044 R_{*}$ (green). Middle row: simulations of a $3.0 R_{\oplus}$ Neptune-sized planet transiting a $4100 \mathrm{~K}, 0.623 R_{\odot} \mathrm{K} 5 \mathrm{~V}$ dwarf star with $i=90.0^{\circ}, P=4 \mathrm{~d}, T_{\text {spot }}=3900 \mathrm{~K}$, and rms scatter $200 \pm 10 \mathrm{ppm}$. Each column on the middle row shows a different $\lambda_{\mathrm{obs}}$, where $\lambda_{\mathrm{obs}}=600,785$, and $1000 \mathrm{~nm}$ (left to right). The smallest detected $r_{\text {spot }}$ values are $r_{\text {spot }}=0.052 R_{*}$ (red), $r_{\text {spot }}=0.061 R_{*}$ (blue), and $r_{\text {spot }}=0.079 R_{*}$ (green). Bottom row: simulations of a $2.25 R_{\oplus}$ sub-Neptune planet transiting a $3700 \mathrm{~K}, 0.493 R_{\odot}$ M1V dwarf star with $i=90.0^{\circ}, T_{\text {spot }}=3550 \mathrm{~K}, \lambda_{\mathrm{obs}}=1000 \mathrm{~nm}$ and rms scatter $100 \pm 5 \mathrm{ppm}$. Each column on the bottom row shows a different $P$, where $P=2 \mathrm{~d}, 3 \mathrm{~d}$ and $4 \mathrm{~d}$ (left to right). The smallest detected $r_{\text {spot }}$ are $r_{\mathrm{spot}}=0.044 R_{*}(\mathrm{red}), r_{\mathrm{spot}}=0.044 R_{*}$ (blue), and $r_{\mathrm{spot}}=0.052 R_{*}$ (green). The solid lines represent the noise-free synthetic light curves containing the starspot anomaly, while the filled circles represent the spot-free synthetic light curves (see Sect. 3) with added Gaussian noise.

Table 3. Comparison between $R_{\mathrm{p}}$ and $\bar{r}_{\text {spot }}$ for the M4V, M1V and K5V host stars.

\begin{tabular}{|c|c|c|c|c|c|}
\hline \multicolumn{2}{|c|}{ M4V } & \multicolumn{2}{|c|}{ M1V } & \multicolumn{2}{|c|}{$\mathrm{K} 5 \mathrm{~V}$} \\
\hline$R_{\mathrm{p}}\left(R_{\oplus}\right)$ & $\bar{r}_{\text {spot }}\left(R_{*}\right)$ & $R_{\mathrm{p}}\left(R_{\oplus}\right)$ & $\bar{r}_{\text {spot }}\left(R_{*}\right)$ & $R_{\mathrm{p}}\left(R_{\oplus}\right)$ & $\bar{r}_{\text {spot }}\left(R_{*}\right)$ \\
\hline 1.00 & $0.042 \pm 0.024$ & 2.00 & $0.045 \pm 0.028$ & 3.0 & $0.044 \pm 0.031$ \\
\hline 1.25 & $0.038 \pm 0.014$ & 2.25 & $0.044 \pm 0.023$ & 3.5 & $0.040 \pm 0.023$ \\
\hline 1.50 & $0.038 \pm 0.012$ & 2.50 & $0.042 \pm 0.021$ & 4.0 & $0.037 \pm 0.017$ \\
\hline 1.75 & $0.038 \pm 0.010$ & 2.75 & $0.042 \pm 0.021$ & 4.5 & $0.035 \pm 0.014$ \\
\hline 2.00 & $0.040 \pm 0.010$ & 3.00 & $0.040 \pm 0.019$ & 5.0 & $0.035 \pm 0.012$ \\
\hline 2.25 & $0.044 \pm 0.010$ & 3.25 & $0.038 \pm 0.016$ & 5.5 & $0.035 \pm 0.012$ \\
\hline 2.50 & $0.045 \pm 0.010$ & 3.50 & $0.037 \pm 0.014$ & 6.0 & $0.035 \pm 0.010$ \\
\hline 2.75 & $0.051 \pm 0.014$ & 3.75 & $0.037 \pm 0.014$ & 6.5 & $0.035 \pm 0.010$ \\
\hline 3.00 & $0.054 \pm 0.014$ & 4.00 & $0.037 \pm 0.012$ & 7.0 & $0.035 \pm 0.010$ \\
\hline
\end{tabular}




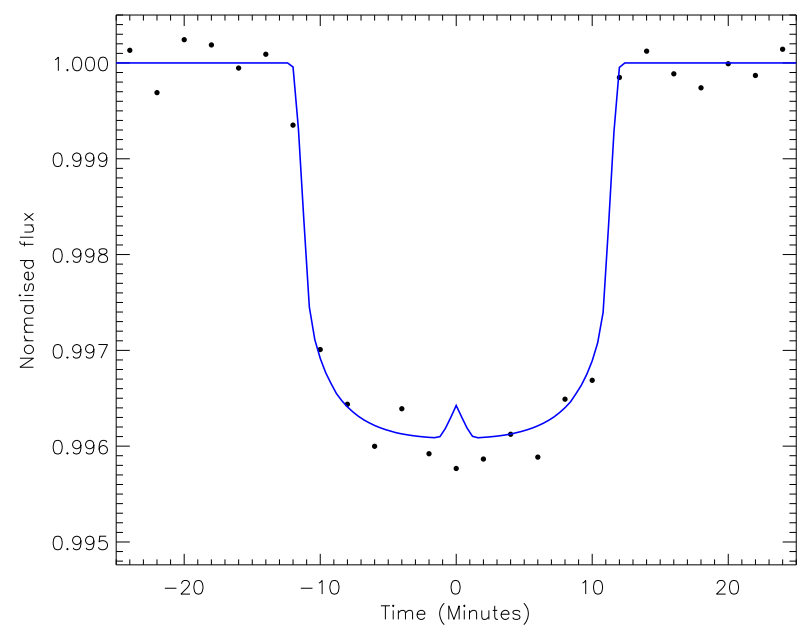

Fig. 9. Example of a single data point describing the apex of a starspot anomaly. Using a $1.0 R_{\oplus}$ Earth-sized planet transiting a $3200 \mathrm{~K}$, $0.155 R_{\odot} \mathrm{M} 4 \mathrm{~V}$ dwarf star with $i=90.0^{\circ}$ and $P=2 \mathrm{~d}$. The starspot properties are $\theta=0^{\circ}, \phi=90^{\circ}, r_{\mathrm{spot}}=0.061 R_{*}$, and $T_{\mathrm{spot}}=3150 \mathrm{~K}$. The observational wavelength and rms scatter for the simulated data were set at $785 \mathrm{~nm}$ and $200 \pm 10 \mathrm{ppm}$, respectively. The solid line represents the noise-free synthetic light curve containing the starspot anomaly, while the filled circles represent the spot-free synthetic light curve (see Sect. 3) with added Gaussian noise.

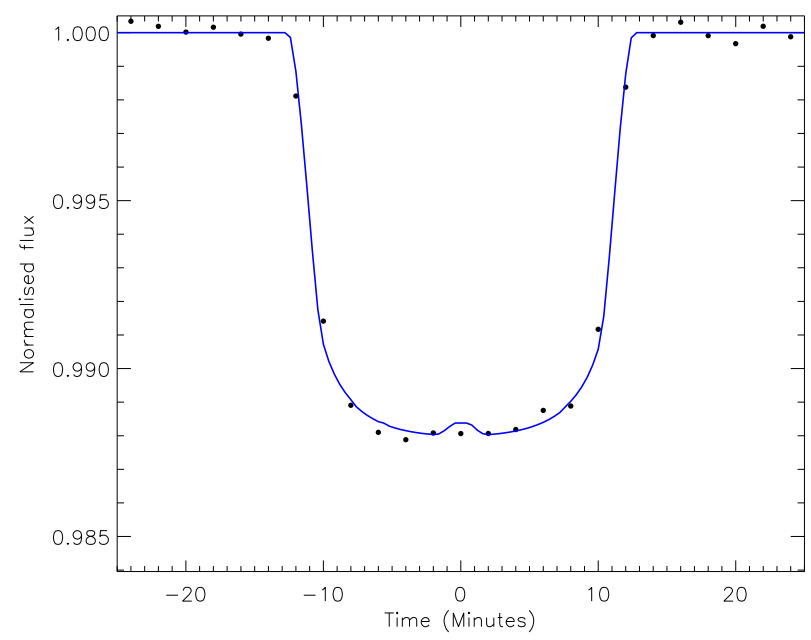

Fig. 10. Example of multiple data points describing the plateau of a starspot anomaly. Using a $1.75 R_{\oplus}$ super-Earth planet transiting a $3200 \mathrm{~K}, 0.155 R_{\odot} \mathrm{M} 4 \mathrm{~V}$ dwarf star with $i=90.0^{\circ}$ and $P=2 \mathrm{~d}$. The starspot properties are $\theta=0^{\circ}, \phi=90^{\circ}, r_{\text {spot }}=0.061 R_{*}$, and $T_{\text {spot }}=$ $3150 \mathrm{~K}$. The observational wavelength and rms scatter for the simulated data were set at $785 \mathrm{~nm}$ and $200 \pm 10 \mathrm{ppm}$, respectively. The solid line represents the noise-free synthetic light curve containing the starspot anomaly, while the filled circles represent the spot-free synthetic light curve (see Sect. 3) with added Gaussian noise.

The mean amplitude of the starspot anomalies are $205 \mathrm{ppm}$ (Fig. 9) and 394 ppm (Fig. 10). This shows how increasing the duration of starspot anomaly can allow for its detection and explains why large planets are needed to detect starspots which are both small and hot in TESS transit light curves.

\subsection{Comparing the change in flux between the planet and starspot}

While the values of $R_{\mathrm{p}}$ and $\bar{r}_{\text {spot }}$ given in Table 3 are valuable they do not allow for a direct comparison between the three
Table 4. Thirty-six values of $\rho_{\text {spot }}$ used in the simulations and the 3 calculated $\bar{\rho}_{\text {spot }}$ for the M4V, M1V, and K5V host star simulations (highlighted in bold).

\begin{tabular}{|c|c|c|c|c|c|c|c|c|}
\hline \multicolumn{3}{|c|}{$\begin{array}{c}\mathrm{M} 4 \mathrm{~V} \\
T_{\text {eff }}=3200 \mathrm{~K}\end{array}$} & \multicolumn{3}{|c|}{$\begin{array}{c}\mathrm{M} 1 \mathrm{~V} \\
T_{\mathrm{eff}}=3700 \mathrm{~K}\end{array}$} & \multicolumn{3}{|c|}{$\begin{array}{c}\mathrm{K} 5 \mathrm{~V} \\
T_{\text {eff }}=4100 \mathrm{~K}\end{array}$} \\
\hline $\begin{array}{l}T_{\text {spot }} \\
(\mathrm{K})\end{array}$ & $\begin{array}{l}\lambda_{\mathrm{obs}} \\
(\mathrm{nm})\end{array}$ & $\rho_{\text {spot }}$ & $\begin{array}{l}T_{\text {spot }} \\
(\mathrm{K})\end{array}$ & $\begin{array}{l}\lambda_{\mathrm{obs}} \\
(\mathrm{nm})\end{array}$ & $\rho_{\text {spot }}$ & $\begin{array}{l}T_{\text {spot }} \\
(\mathrm{K})\end{array}$ & $\begin{array}{l}\lambda_{\mathrm{obs}} \\
(\mathrm{nm})\end{array}$ & $\rho_{\text {spot }}$ \\
\hline \multirow{3}{*}{3000} & 600 & 0.61 & \multirow{3}{*}{3400} & 600 & 0.56 & \multirow{3}{*}{3700} & 600 & 0.53 \\
\hline & 785 & 0.68 & & 785 & 0.64 & & 785 & 0.61 \\
\hline & 1000 & 0.74 & & 1000 & 0.70 & & 1000 & 0.68 \\
\hline \multirow{3}{*}{3050} & 600 & 0.69 & \multirow{3}{*}{3475} & 600 & 0.66 & \multirow{3}{*}{3800} & 600 & 0.63 \\
\hline & 785 & 0.75 & & 785 & 0.72 & & 785 & 0.70 \\
\hline & 1000 & 0.80 & & 1000 & 0.77 & & 1000 & 0.75 \\
\hline \multirow{3}{*}{3100} & 600 & 0.78 & \multirow{3}{*}{3550} & 600 & 0.76 & \multirow{3}{*}{3900} & 600 & 0.74 \\
\hline & 785 & 0.83 & & 785 & 0.81 & & 785 & 0.79 \\
\hline & 1000 & 0.86 & & 1000 & 0.85 & & 1000 & 0.83 \\
\hline \multirow{4}{*}{3150} & 600 & 0.89 & \multirow{3}{*}{3625} & 600 & 0.87 & \multirow{3}{*}{4000} & 600 & 0.86 \\
\hline & 785 & 0.91 & & 785 & 0.90 & & 785 & 0.89 \\
\hline & 1000 & 0.93 & & 1000 & 0.92 & & 1000 & 0.91 \\
\hline & & 0.79 & & & 0.76 & & & 0.74 \\
\hline
\end{tabular}

host star spectral types. To allow a direct comparison, $R_{\mathrm{p}}$ and $\bar{r}_{\text {spot }}$ need to be converted into change in flux $(\Delta F) . R_{\mathrm{p}}$ can be converted to $\Delta F_{\mathrm{p}}$ using $k^{2}$, where

$$
\begin{aligned}
\Delta F_{\mathrm{p}} & =k^{2} \\
& =\left(\frac{R_{\mathrm{p}}}{R_{*}}\right)^{2},
\end{aligned}
$$

which gives the area of the planetary disc in units of a uniform stellar disc. Because the simulations used in this work examined starspot occultations when the starspot was at the centre of the stellar disc, we can use this approximation of $\Delta F_{\mathrm{p}}$ in the absence of limb darkening.

The surface area of the starspot is the solid angle, $\Omega$, and can be determined from $r_{\text {spot }}$ (in units of angular radius) using

$\Omega=2 \pi R_{*}^{2}\left(1-\cos r_{\text {spot }}\right)$,

which in units of the stellar hemisphere $\left(2 \pi R_{*}^{2}\right)$ becomes

$\frac{\Omega}{2 \pi R_{*}^{2}}=\left(1-\cos r_{\text {spot }}\right)$.

However, unlike the planet, the starspot emits a flux which means that the flux deficit caused by the starspot, $\Delta F_{\text {spot }}$, is related to $r_{\text {spot }}$ by $\rho_{\text {spot }}$ using the following relation:

$\Delta F_{\text {spot }}=\left(1-\cos r_{\text {spot }}\right)\left(1-\rho_{\text {spot }}\right)$.

For the simulations in this work 36 different values of $\rho_{\text {spot }}$ were used. These were derived using Eq. (2) using three values of $T_{\text {eff }}$, four $T_{\text {spot }}$, and three $\lambda_{\text {obs }}$. This equates to 12 different values of $\rho_{\text {spot }}$ for each stellar spectral class. To determine $\Delta F_{\text {spot }}$, the mean starspot contrast $\left(\bar{\rho}_{\text {spot }}\right)$ was calculated for each spectral class and are given in Table 4.

Therefore $\Delta F_{\text {spot }}$ was calculated for the nine $\bar{r}_{\text {spot }}$ (Table 3) for each spectral class using the associated $\bar{\rho}_{\text {spot }}$ (Table 4$)$. The 


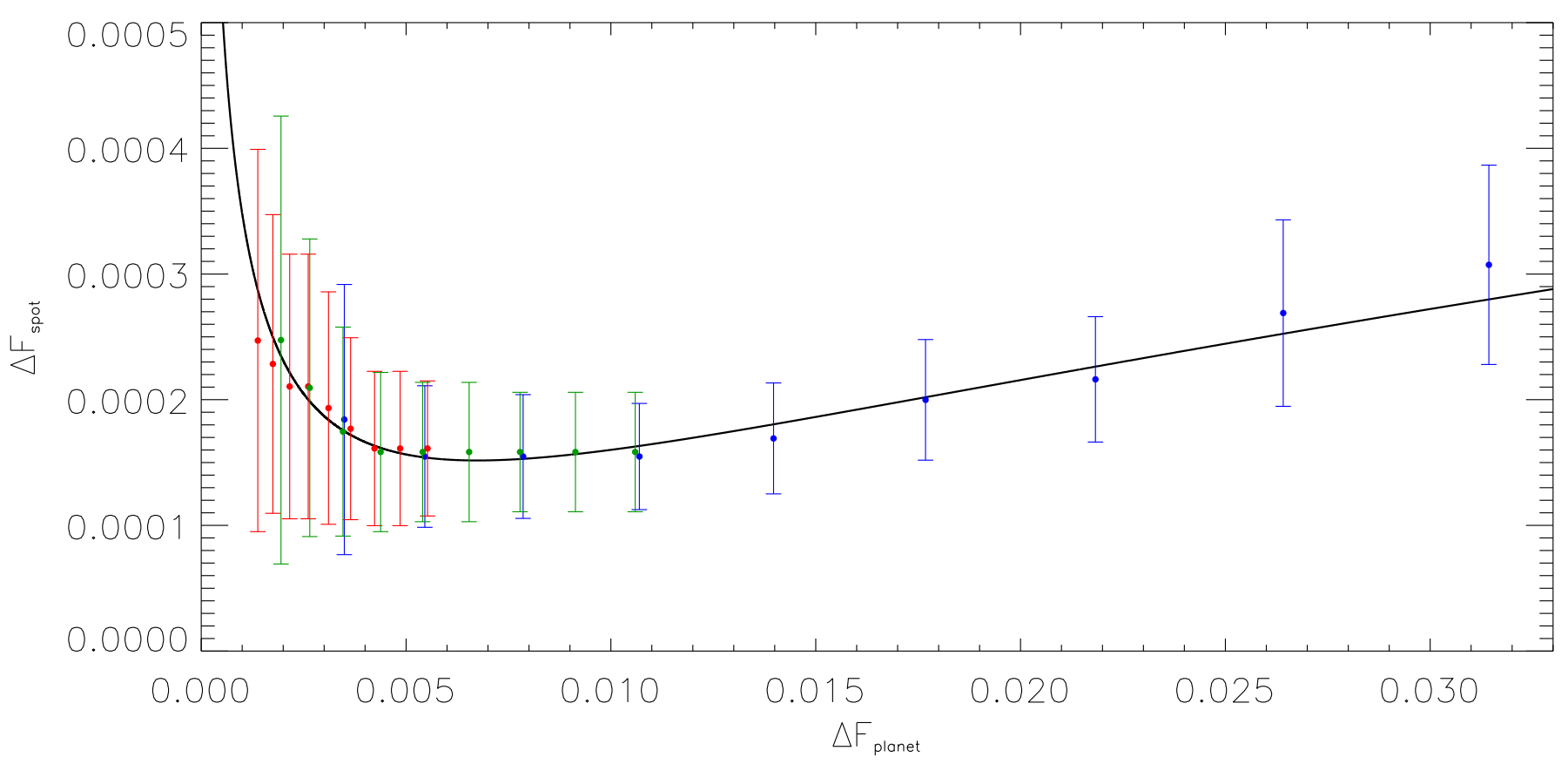

Fig. 11. Plot of $\Delta F_{\mathrm{p}}$ and $\Delta F_{\text {spot }}$ calculated from Eq. (4) and (7) combined with the best fitting model (solid black line). The blue data points represent the M4V host star results. The red data points represent the M1V host star results, while the green data points represent the K5V host star results.

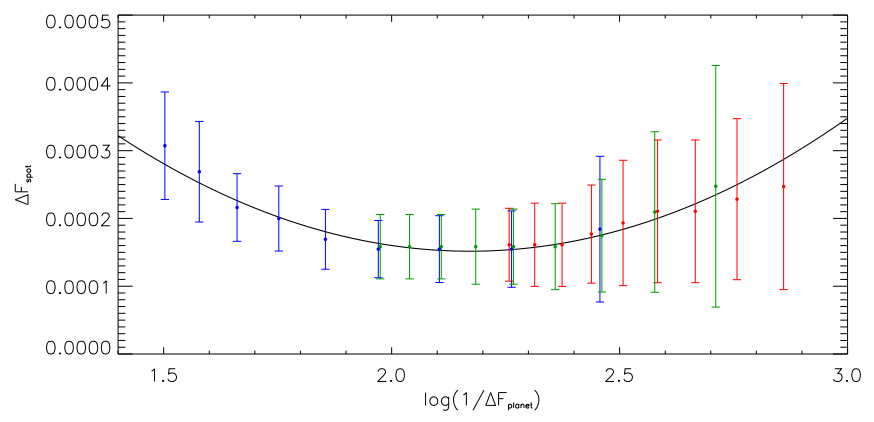

Fig. 12. Plot of $\log _{10}\left(\frac{1}{\Delta F_{\mathrm{p}}}\right)$ and $\Delta F_{\text {spot }}$ calculated from Eqs. (4) and (7) combined with the best fitting model (solid black line). The blue data points represent the M4V host star results. The red data points represent the M1V host star results, while the green data points represent the K5V host star results.

resultant calculated values of $\Delta F_{\mathrm{p}}$ (Eq. (4)) and $\Delta F_{\text {spot }}$ (Eq. (7)) are given in Figs. 11 and 12.

Figure 11 shows that the results for each of the three host stars are in excellent agreement with each other in terms of the smallest $\Delta F_{\text {spot }}$ that can be detected. The simulation results indicate that when $k \geq 0.10$ a starspot needs a larger $\Delta F_{\text {spot }}$ to be detected, either by increasing $r_{\text {spot }}$ or by decreasing $\rho_{\text {spot }}$. The positive gradient towards larger $\Delta F_{\mathrm{p}}$ represents that larger planets can only detect larger or cooler starspots. The steep negative gradient towards smaller $\Delta F_{\mathrm{p}}$ shows that the effect from using a fixed cadence, combined with the method that is used to detect starspot anomalies, has a stronger influence on the size of starspot which can be detected in TESS transit light curves.

To determine which $\Delta F_{\mathrm{p}}$ allows for the smallest $\Delta F_{\text {spot }}$ to be detected, we fitted a parametrised second order polynomial to the results. This was accomplished by parametrising $\Delta F_{\mathrm{p}}$ to a logarithmic scale given by

$t=\log _{10}\left(\frac{1}{\Delta F_{\mathrm{p}}}\right)$,

where $t$ is the logarithmic parametrisation of $\Delta F_{\mathrm{p}}$.

Figure 12 shows the comparison between $t$ and $\Delta F_{\text {spot }}$, which indicates a quadratic relation between the two quantities. We used Markov chain Monte Carlo to fit a quadratic relation as follows:

$$
\begin{aligned}
\Delta F_{\text {spot }}= & (0.00028 \pm 0.00001) t^{2} \\
& -(0.00121 \pm 0.00001) t \\
& +(0.00147 \pm 0.00002) .
\end{aligned}
$$

Using Eq. (9) and setting $\frac{\mathrm{d} \Delta F_{\text {spot }}}{\mathrm{d} t}=0$ we find that the smallest detectable $\Delta F_{\text {spot }}=0.00015 \pm 0.00001$, when $\Delta F_{\mathrm{p}}=0.0067 \pm$ $0.0006(k=0.082 \pm 0.004)$. This allows us to determine that for a $1 R_{\oplus}$ planet, a host star with $R_{*}=0.112 \pm 0.005 R_{\odot}$ is required to detect starspots with $\Delta F_{\text {spot }}=0.00015 \pm 0.00001$. While for a $3 R_{\oplus}$ sub-Neptune planet, a host star with $R_{*}=0.335 \pm 0.015 R_{\odot}$ is required to find starspots with $\Delta F_{\text {spot }}=0.00015 \pm 0.00001$.

In Sect. 2.2 we describe how a degeneracy exists between $r_{\text {spot }}$ and $\rho_{\text {spot }}$. For the simulations performed in this work, the smallest $r_{\text {spot }}$ was determined for sets of fixed parameter values, which included $\rho_{\text {spot }}$ (calculated from $T_{\text {spot }}, T_{\text {eff }}$, and $\lambda_{\text {obs }}$ using Eq. (2)). Table 4 gives the range of $\rho_{\text {spot }}$ used in the simulations (0.53-0.93) and when combined with the results given in Tables A.1-A.3 allows an extrapolation to determine the largest $\rho_{\text {spot }}$ for a fixed $r_{\text {spot }}$.

However, Eq. (9) can be used to determine the largest average detectable $\rho_{\text {spot }}$ for a fixed $r_{\text {spot }}$. For a $1 R_{\text {Jup }}$ planet, transiting a $1 R_{\odot}$ star, the change in flux caused by the transiting planet is $\Delta F_{\mathrm{p}} \approx 0.01$. When parametrised using Eq. (8) gives $t=2$. Equation (9) then calculates the smallest detectable $\Delta F_{\text {spot }}$ for the system, $\Delta F_{\text {spot }}=0.000166 \pm 0.00006$. Using Eq. (7) with a 
given starspot angular radius, $r_{\mathrm{spot}}=5^{\circ}\left(r_{\mathrm{spot}}=0.087 R_{*}\right)$ we can determine the lowest contrast detectable in TESS transit light curves for the starspot, $\rho_{\text {spot }}=0.96 \pm 0.02$. While for a $r_{\text {spot }}=2^{\circ}$ $\left(r_{\text {spot }}=0.035 R_{*}\right)$ we find the lowest contrast detectable to be $\rho_{\text {spot }}=0.73 \pm 0.10$. For a $r_{\text {spot }}=1^{\circ}\left(r_{\text {spot }}=0.017 R_{*}\right)$ Eq. $(7)$ gives a negative value for $\rho_{\text {spot }}$, indicating that it is not possible for a $r_{\text {spot }}=0.017 R_{*}$ starspot to be detected in TESS transit data of a $1 R_{\text {Jup }}$ planet that is transiting a $1 R_{\odot}$ star.

\section{Discussion and conclusions}

The key result from the simulations performed in this work indicates that when TESS observes a transiting planet that occults a starspot on the stellar disc, the characteristic blip of the starspot anomaly is seen in the resulting light curve. This removes any doubt that starspot anomalies are present in TESS light curves because the TESS primary targets are cooler (redder) host stars with shorter transit durations. It also opens the possibility of using the starspot tracking technique (e.g. Tregloan-Reed et al. 2013, 2015; Mohler-Fischer et al. 2013; Southworth et al. 2016; Mancini et al. 2017) to measure the orbital obliquity of TESS planetary systems. Particularly for targets where the conventional Rossiter-McLaughlin technique (Rossiter 1924; McLaughlin 1924; Schlesinger 1910, 1916; Holt 1893 ) is not viable (e.g. slow-rotators, small $k$, and active stars). The simulations then go further in determining the detection limits for a variety of starspot temperatures, planetary and stellar radii, photometric precision, orbital periods, and observational wavelengths. It was found that the starspot detection limits in TESS light curves for $\mathrm{M} 4 \mathrm{~V}, \mathrm{M} 1 \mathrm{~V}$, and $\mathrm{K} 5 \mathrm{~V}$ host stars were $4900 \pm 1700,13800 \pm 6000$, and $15900 \pm 6800 \mathrm{~km}$, respectively.

The simulations confirmed expected trends in the detection limits of starspot anomalies, including the photometric parameter dependencies of the amplitude of the starspot anomaly. The results confirmed that the amplitude of a starspot anomaly is strongly dependent on $r_{\text {spot }}$ and $\rho_{\text {spot }}$, and therefore, by association with $\rho_{\text {spot }}$, strongly dependent on $T_{\text {eff }}$ and $\lambda_{\text {obs }}$. While, the amplitude of the starspot anomaly is less dependent on $P$. The simulations then show that overall the detection limit of a starspot anomaly is dependent on $R_{\mathrm{p}}$ (Table 3) and is therefore dependent on $\Delta F_{\text {planet }}$. However, the simulations uncovered an unexpected trend: starspots that are small and hot can be detected by large planets, however, they cannot be detected by smaller planets.

From our investigations of the synthetic light curves, we uncovered that the unexpected trend was a detection bias generated by the fixed 2 min cadence of TESS combined with the method used to determine if the starspot anomaly was detectable, by comparing the mean amplitude of a starspot anomaly to the observational noise. This detection method is in agreement with fitting starspot anomalies in transit light curves. If the mean amplitude of the starspot anomaly is not large enough, it is not possible to constrain the starspot properties (see Sect. 3). It was found that for anomalies with a short maximum duration (apex shape) the data points lying on the ingress and egress of the starspot anomaly reduced the mean amplitude. As a consequence for the starspot to be detected either $r_{\text {spot }}$ or $R_{\mathrm{p}}$ needed to be increased to increase the mean amplitude. Although as in the case depicted in Fig. 4, if the maximum amplitude of the starspot anomaly is below the detection threshold, then increasing $r_{\text {spot }}$ further did not allow a detection. If the maximum amplitude was tested against the rms scatter in the data instead of using the mean amplitude, this would allow starspot anomalies described by a single data point (i.e. apex shape) to be considered detected when in reality these anomalies would be dismissed as noise (e.g. Fig. 9). Using the mean amplitude of the starspot anomaly effectively mimics what happens when a transit-starspot model fits a starspot anomaly. Hence, the unexpected trend that smaller hot starspots are detected by larger planets will be seen when analysing TESS transit light curves. To counter this would require a reduction in the observational cadence, which would increase the number of data points that describe the starspot anomaly. However, this is not practical when analysing TESS transit data and would require further follow-up photometric observations using a different telescope.

By separating the simulations into three groups for each of the three host stars and then converting $R_{\mathrm{p}}$ and $\bar{r}_{\text {spot }}$ into $\Delta F_{\text {planet }}$ and $\Delta F_{\text {spot }}$, a direct comparison between the planets and starspots for the three host stars was made. The resultant comparison between $\Delta F_{\text {planet }}$ and $\Delta F_{\text {spot }}$ shows excellent agreement of the simulations between the three host stars and indicates that the smallest and hottest starspots detected in TESS transit light curves are when $k=0.082 \pm 0.004$. By fitting a parametrised second order polynomial to the results, we are able to determine the minimum size and lowest contrast (highest intensity) that can be detected for a given value of $k$ in the TESS exoplanetary transit data. The comparison also revealed that the effect from using a fixed 2 min cadence combined with the mean flux of a starspot anomaly to determine its detection had a larger impact on $\Delta F_{\text {spot }}$ than $R_{\mathrm{p}}$.

Young $\mathrm{K}$ and $\mathrm{M}$ dwarf stars are known to have a high starspot surface coverage (e.g. O’Neal et al. 2004; Jackson \& Jeffries 2013). However, the activity-age relationship is still unknown. This leads to the question of how many TESS transit light curves will contain starspot anomalies. The simulations presented in this work are the starting point of answering this question in that the simulations show that starspot anomalies are observable in TESS transit light curves. The starspot distribution on the stellar disc is also an important factor in this question. Barnes et al. (2015) used high resolution Doppler images of two $\mathrm{M}$ dwarfs (GJ 791.2 A; M4.5: LP 944-20; M9) to determine the overall starspot filling factors $\approx 3$ and $\approx 1.5 \%$ for the two $\mathrm{M}$ dwarfs. However, Barnes et al. (2015) found maximum starspot filling factors 82.3 and $76.6 \%$ at high latitudes (polar). If the starspot distributions of GJ 791.2 A and LP 944-20 can be considered typical of $\mathrm{M}$ dwarf stars that will be observed by TESS, then a polar biased starspot distribution will affect the total number of TESS transit light curves which contain a starspot anomaly. Determining the smallest detectable starspot size for a range of planetary sizes, starspot temperatures, orbital periods, and noise levels is an essential first step in beginning to forecast the number of TESS transit light curves which will be affected by starspot anomalies. Therefore, these studies will gauge the importance and the future reliance by the community on transit-starspot models.

Acknowledgements. We would like to thank the anonymous referee and the editor for their helpful comments, which improved the quality of this manuscript. This work was supported by a CONICYT/FONDECYT 2018 Postdoctoral research grant, project number: 3180071. J.T.R. thanks the Centro de Astronomía (CITEVA), Universidad de Antofagasta for hosting the CONICYT/FONDECYT 2018 Postdoctoral research grant. The following internet-based resources were used in the research for this paper: the NASA Astrophysics Data System; the SIMBAD database and VizieR catalogue access tool operated at CDS, Strasbourg, France, and the arXiv scientific paper preprint service operated by Cornell University.

\section{References}

Ballerini, P., Micela, G., Lanza, A. F., \& Pagano, I. 2012, A\&A, 539, A140 Barnes, J. R., Jeffers, S. V., Jones, H. R. A., et al. 2015, ApJ, 812, 42 
Bayliss, D., Gillen, E., Eigmüller, P., et al. 2018, MNRAS, 475, 4467 Béky, B., Kipping, D. M., \& Holman, M. J. 2014, MNRAS, 442, 3686 Borucki, W. J., Koch, D., Basri, G., et al. 2010, Science, 327, 977 Boyajian, T. S., von Braun, K., van Belle, G., et al. 2012, ApJ, 757, 112 Chen, G., Guenther, E. W., Pallé, E., et al. 2017, A\&A, 600, A138 Claret, A. 2017, A\&A, 600, A30

Dotter, A., Chaboyer, B., Jevremović, D., et al. 2008, ApJS, 178, 89

Dragomir, D., Teske, J., Gunther, M. N., et al. 2019, ApJ, 875, L7

Dressing, C. D., \& Charbonneau, D. 2013, ApJ, 767, 95

Dressing, C. D., \& Charbonneau, D. 2015, ApJ, 807, 45

Fortney, J. J., Marley, M. S., \& Barnes, J. W. 2007, ApJ, 659, 1661

Hartman, J. D., Bayliss, D., Brahm, R., et al. 2015, AJ, 149, 166

Herrero, E., Ribas, I., Jordi, C., et al. 2016, A\&A, 586, A131

Holt, J. R. 1893, Astronomy and Astro-Physics, 12, 646

Huang, C. X., Burt, J., Vanderburg, A., et al. 2018, ApJ, 868, L39

Jackson, R. J., \& Jeffries, R. D. 2013, MNRAS, 431, 1883

Jenkins, J. M., Twicken, J. D., McCauliff, S., et al. 2016, Proc. SPIE, 9913, 99133E

Johnson, J. A., Gazak, J. Z., Apps, K., et al. 2012, AJ, 143, 111

Juvan, I. G., Lendl, M., Cubillos, P. E., et al. 2018, A\&A, 610, A15

Kopparapu, R. K., Ramirez, R., Kasting, J. F., et al. 2013, ApJ, 765, 131

Mancini, L., Ciceri, S., Chen, G., et al. 2013, MNRAS, 436, 2

Mancini, L., Southworth, J., Ciceri, S., et al. 2014, MNRAS, 443, 2391

Mancini, L., Esposito, M., Covino, E., et al. 2015, A\&A, 579, A136

Mancini, L., Southworth, J., Raia, G., et al. 2017, MNRAS, 465, 843

Maxted, P. F. L. 2016, A\&A, 591, A111

McLaughlin, D. B. 1924, ApJ, 60, 22

Mohler-Fischer, M., Mancini, L., Hartman, J. D., et al. 2013, A\&A, 558, A55

Montalto, M., Boué, G., Oshagh, M., et al. 2014, MNRAS, 444, 1721

Morton, T. D., \& Swift, J. 2014, ApJ, 791, 10

Nikolov, N., Chen, G., Fortney, J. J., et al. 2013, A\&A, 553, A26

O'Neal, D., Neff, J. E., Saar, S. H., \& Cuntz, M. 2004, AJ, 128, 1802

Oshagh, M., Boisse, I., Boué, G., et al. 2013, A\&A, 549, A35

Prša, A., \& Zwitter, T. 2005, ApJ, 628, 426

Prša, A., Conroy, K. E., Horvat, M., et al. 2016, ApJS, 227, 29

Rabus, M., Alonso, R., Belmonte, J. A., et al. 2009, A\&A, 494, 391

Ricker, G. R., Latham, D. W., Vanderspek, R. K., et al. 2009, BAAS, 41, 193

Ricker, G. R., Winn, J. N., Vanderspek, R., et al. 2014, Proc. SPIE, 9143, 914320

Ricker, G. R., Winn, J. N., Vanderspek, R., et al. 2015, J. Astron. Telesc. Instrum. Syst., 1, 014003

Rossiter, R. A. 1924, ApJ, 60, 15
Sanchis-Ojeda, R., \& Winn, J. N. 2011, ApJ, 743, 61

Sanchis-Ojeda, R., Winn, J. N., Holman, M. J., et al. 2011, ApJ, 733, 127

Sanchis-Ojeda, R., Fabrycky, D. C., Winn, J. N., et al. 2012, Nature, 487, 449

Sanchis-Ojeda, R., Winn, J. N., Marcy, G. W., et al. 2013, ApJ, 775, 54

Schlesinger, F. 1910, Publications of the Allegheny Observatory of the University of Pittsburgh (Charleston: Nabu Press), 1, 123

Schlesinger, F. 1916, Publications of the Allegheny Observatory of the University of Pittsburgh (Charleston: Nabu Press), 3, 23

Silva, A. V. R. 2003, ApJ, 585, L147

Silva-Válio, A. 2010, IAU Symp., 264, 440

Silva-Valio, A., Lanza, A. F., Alonso, R., \& Barge, P. 2010, A\&A, 510, A25

Southworth, J. 2008, MNRAS, 386, 1644

Southworth, J. 2010, MNRAS, 408, 1689

Southworth, J. 2011, MNRAS, 417, 2166

Southworth, J. 2013, A\&A, 557, A119

Southworth, J., Maxted, P. F. L., \& Smalley, B. 2004, MNRAS, 351, 1277

Southworth, J., Smalley, B., Maxted, P. F. L., Claret, A., \& Etzel, P. B. 2005 , MNRAS, 363, 529

Southworth, J., Bruntt, H., \& Buzasi, D. L. 2007, A\&A, 467, 1215

Southworth, J., Hinse, T. C., Dominik, M., et al. 2009, ApJ, 707, 167

Southworth, J., Tregloan-Reed, J., Andersen, M. I., et al. 2016, MNRAS, 457, 4205

Stassun, K. G., Oelkers, R. J., Pepper, J., et al. 2018, AJ, 156, 102

Sullivan, P. W., Winn, J. N., Berta-Thompson, Z. K., et al. 2015, ApJ, 809, 77

Sullivan, P. W., Winn, J. N., Berta-Thompson, Z. K., et al. 2017, ApJ, 837, 99

Tregloan-Reed, J., Southworth, J., \& Tappert, C. 2013, MNRAS, 428, 3671

Tregloan-Reed, J., Southworth, J., Burgdorf, M., et al. 2015, MNRAS, 450, 1760

Tregloan-Reed, J., Southworth, J., Mancini, L., et al. 2018, MNRAS, 474, 5485

Valio, A., Estrela, R., Netto, Y., Bravo, J. P., \& de Medeiros, J. R. 2017, ApJ, 835, 294

Vanderspek, R., Huang, C. X., Vanderburg, A., et al. 2019, ApJ, 871, L24

Wang, S., Jones, M., Shporer, A., et al. 2019, AJ, 157, 51

Wheatley, P. J., Pollacco, D. L., Queloz, D., et al. 2013, Eur. Phys. J. Web Conf., 47, 13002

Wilson, R. E. 1979, ApJ, 234, 1054

Wilson, R. E. 1990, ApJ, 356, 613

Wilson, R. E. 2008, ApJ, 672, 575

Wilson, R. E. 2012, AJ, 144, 73

Wilson, R. E., \& Devinney, E. J. 1971, ApJ, 166, 605

Winn, J. N. 2010, Exoplanet Transits and Occultations, ed. S. Seager (Tucson, AZ: University of Arizona Press), 55 


\section{Appendix A: Full results of all simulations}

Table A.1. Smallest detectable value of $r_{\text {spot }}$ determined from the 6718 simulations of the 1296 scenarios that used an M4V dwarf host star.

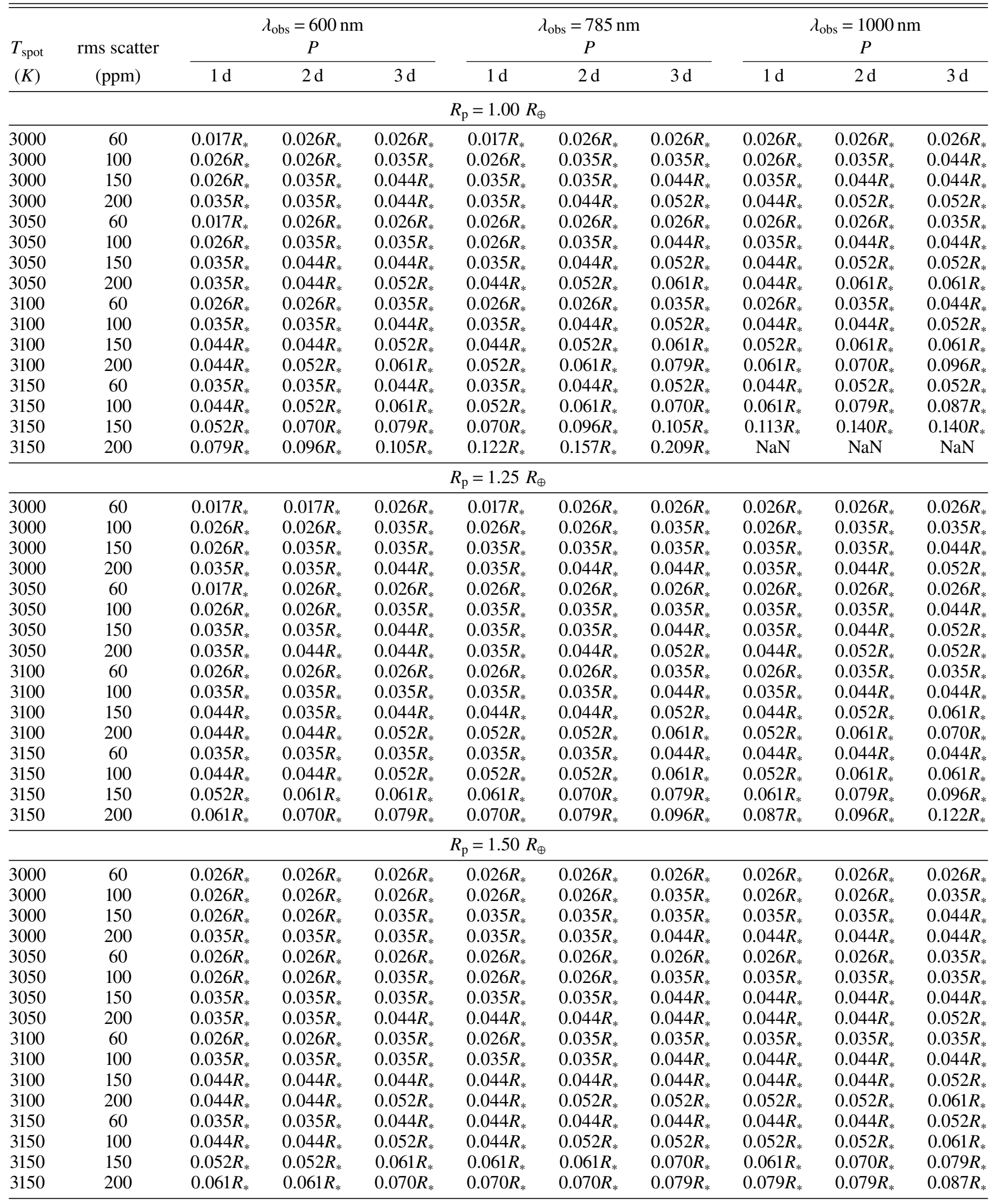


Table A.1. continued.

\begin{tabular}{|c|c|c|c|c|c|c|c|c|c|c|}
\hline \multirow{2}{*}{$\begin{array}{c}T_{\text {spot }} \\
(K)\end{array}$} & \multirow{2}{*}{$\begin{array}{l}\text { rms scatter } \\
(\mathrm{ppm})\end{array}$} & \multicolumn{3}{|c|}{$\begin{array}{c}\lambda_{\mathrm{obs}}=600 \mathrm{~nm} \\
P\end{array}$} & \multicolumn{3}{|c|}{$\begin{array}{c}\lambda_{\mathrm{obs}}=785 \mathrm{~nm} \\
P\end{array}$} & \multicolumn{3}{|c|}{$\begin{array}{c}\lambda_{\mathrm{obs}}=1000 \mathrm{~nm} \\
P\end{array}$} \\
\hline & & $1 \mathrm{~d}$ & $2 \mathrm{~d}$ & $3 d$ & $1 \mathrm{~d}$ & $2 d$ & $3 d$ & $1 \mathrm{~d}$ & $2 \mathrm{~d}$ & $3 d$ \\
\hline \multicolumn{11}{|c|}{$R_{\mathrm{p}}=1.75 R_{\oplus}$} \\
\hline $\begin{array}{l}3000 \\
3000 \\
3000 \\
3000 \\
3050 \\
3050 \\
3050 \\
3050 \\
3100 \\
3100 \\
3100 \\
3100 \\
3150 \\
3150 \\
3150 \\
3150\end{array}$ & $\begin{array}{c}60 \\
100 \\
150 \\
200 \\
60 \\
100 \\
150 \\
200 \\
60 \\
100 \\
150 \\
200 \\
60 \\
100 \\
150 \\
200\end{array}$ & $\begin{array}{c}0.026 R_{*} \\
0.026 R_{*} \\
0.026 R_{*} \\
0.035 R_{*} \\
0.026 R_{*} \\
0.026 R_{*} \\
0.035 R_{*} \\
0.035 R_{*} \\
0.035 R_{*} \\
0.035 R_{*} \\
0.035 R_{*} \\
0.044 R_{*} \\
0.044 R_{*} \\
0.044 R_{*} \\
0.052 R_{*} \\
0.052 R_{*}\end{array}$ & $\begin{array}{l}0.026 R_{*} \\
0.026 R_{*} \\
0.026 R_{*} \\
0.035 R_{*} \\
0.026 R_{*} \\
0.026 R_{*} \\
0.035 R_{*} \\
0.035 R_{*} \\
0.035 R_{*} \\
0.035 R_{*} \\
0.035 R_{*} \\
0.044 R_{*} \\
0.044 R_{*} \\
0.044 R_{*} \\
0.052 R_{*} \\
0.061 R_{*}\end{array}$ & $\begin{array}{c}0.026 R_{*} \\
0.026 R_{*} \\
0.026 R_{*} \\
0.035 R_{*} \\
0.026 R_{*} \\
0.026 R_{*} \\
0.035 R_{*} \\
0.035 R_{*} \\
0.035 R_{*} \\
0.035 R_{*} \\
0.035 R_{*} \\
0.044 R_{*} \\
0.044 R_{*} \\
0.044 R_{*} \\
0.052 R_{*} \\
0.061 R_{*}\end{array}$ & $\begin{array}{l}0.026 R_{*} \\
0.026 R_{*} \\
0.035 R_{*} \\
0.035 R_{*} \\
0.026 R_{*} \\
0.026 R_{*} \\
0.035 R_{*} \\
0.044 R_{*} \\
0.035 R_{*} \\
0.035 R_{*} \\
0.044 R_{*} \\
0.044 R_{*} \\
0.044 R_{*} \\
0.044 R_{*} \\
0.052 R_{*} \\
0.061 R_{*}\end{array}$ & $\begin{array}{l}0.026 R_{*} \\
0.026 R_{*} \\
0.035 R_{*} \\
0.035 R_{*} \\
0.026 R_{*} \\
0.026 R_{*} \\
0.035 R_{*} \\
0.044 R_{*} \\
0.035 R_{*} \\
0.035 R_{*} \\
0.044 R_{*} \\
0.052 R_{*} \\
0.044 R_{*} \\
0.052 R_{*} \\
0.052 R_{*} \\
0.061 R_{*}\end{array}$ & $\begin{array}{c}0.026 R_{*} \\
0.026 R_{*} \\
0.035 R_{*} \\
0.035 R_{*} \\
0.026 R_{*} \\
0.026 R_{*} \\
0.035 R_{*} \\
0.044 R_{*} \\
0.035 R_{*} \\
0.035 R_{*} \\
0.044 R_{*} \\
0.052 R_{*} \\
0.052 R_{*} \\
0.052 R_{*} \\
0.061 R_{*} \\
0.070 R_{*}\end{array}$ & $\begin{array}{c}0.026 R_{*} \\
0.026 R_{*} \\
0.035 R_{*} \\
0.035 R_{*} \\
0.035 R_{*} \\
0.035 R_{*} \\
0.035 R_{*} \\
0.044 R_{*} \\
0.035 R_{*} \\
0.035 R_{*} \\
0.044 R_{*} \\
0.052 R_{*} \\
0.052 R_{*} \\
0.052 R_{*} \\
0.061 R_{*} \\
0.070 R_{*}\end{array}$ & $\begin{array}{c}0.026 R_{*} \\
0.026 R_{*} \\
0.035 R_{*} \\
0.044 R_{*} \\
0.035 R_{*} \\
0.035 R_{*} \\
0.035 R_{*} \\
0.044 R_{*} \\
0.035 R_{*} \\
0.035 R_{*} \\
0.044 R_{*} \\
0.052 R_{*} \\
0.052 R_{*} \\
0.052 R_{*} \\
0.070 R_{*} \\
0.079 R_{*}\end{array}$ & $\begin{array}{c}0.026 R_{*} \\
0.026 R_{*} \\
0.035 R_{*} \\
0.044 R_{*} \\
0.035 R_{*} \\
0.035 R_{*} \\
0.044 R_{*} \\
0.044 R_{*} \\
0.044 R_{*} \\
0.044 R_{*} \\
0.052 R_{*} \\
0.061 R_{*} \\
0.052 R_{*} \\
0.061 R_{*} \\
0.070 R_{*} \\
0.079 R_{*}\end{array}$ \\
\hline \multicolumn{11}{|c|}{$R_{\mathrm{p}}=2.00 R_{\oplus}$} \\
\hline $\begin{array}{l}3000 \\
3000 \\
3000 \\
3000 \\
3050 \\
3050 \\
3050 \\
3050 \\
3100 \\
3100 \\
3100 \\
3100 \\
3150 \\
3150 \\
3150 \\
3150\end{array}$ & $\begin{array}{c}60 \\
100 \\
150 \\
200 \\
60 \\
100 \\
150 \\
200 \\
60 \\
100 \\
150 \\
200 \\
60 \\
100 \\
150 \\
200\end{array}$ & $\begin{array}{c}0.026 R_{*} \\
0.026 R_{*} \\
0.026 R_{*} \\
0.035 R_{*} \\
0.026 R_{*} \\
0.026 R_{*} \\
0.035 R_{*} \\
0.035 R_{*} \\
0.035 R_{*} \\
0.035 R_{*} \\
0.035 R_{*} \\
0.044 R_{*} \\
0.044 R_{*} \\
0.044 R_{*} \\
0.052 R_{*} \\
0.052 R_{*}\end{array}$ & $\begin{array}{l}0.026 R_{*} \\
0.026 R_{*} \\
0.026 R_{*} \\
0.035 R_{*} \\
0.026 R_{*} \\
0.026 R_{*} \\
0.035 R_{*} \\
0.035 R_{*} \\
0.035 R_{*} \\
0.035 R_{*} \\
0.035 R_{*} \\
0.044 R_{*} \\
0.044 R_{*} \\
0.044 R_{*} \\
0.052 R_{*} \\
0.061 R_{*}\end{array}$ & $\begin{array}{c}0.026 R_{*} \\
0.026 R_{*} \\
0.026 R_{*} \\
0.035 R_{*} \\
0.026 R_{*} \\
0.026 R_{*} \\
0.026 R_{*} \\
0.035 R_{*} \\
0.035 R_{*} \\
0.035 R_{*} \\
0.035 R_{*} \\
0.044 R_{*} \\
0.052 R_{*} \\
0.052 R_{*} \\
0.052 R_{*} \\
0.061 R_{*}\end{array}$ & $\begin{array}{l}0.026 R_{*} \\
0.026 R_{*} \\
0.035 R_{*} \\
0.035 R_{*} \\
0.035 R_{*} \\
0.035 R_{*} \\
0.035 R_{*} \\
0.044 R_{*} \\
0.035 R_{*} \\
0.035 R_{*} \\
0.044 R_{*} \\
0.044 R_{*} \\
0.052 R_{*} \\
0.052 R_{*} \\
0.052 R_{*} \\
0.061 R_{*}\end{array}$ & $\begin{array}{l}0.026 R_{*} \\
0.026 R_{*} \\
0.035 R_{*} \\
0.035 R_{*} \\
0.035 R_{*} \\
0.035 R_{*} \\
0.035 R_{*} \\
0.035 R_{*} \\
0.035 R_{*} \\
0.035 R_{*} \\
0.044 R_{*} \\
0.044 R_{*} \\
0.052 R_{*} \\
0.052 R_{*} \\
0.052 R_{*} \\
0.070 R_{*}\end{array}$ & $\begin{array}{c}0.026 R_{*} \\
0.026 R_{*} \\
0.026 R_{*} \\
0.035 R_{*} \\
0.035 R_{*} \\
0.035 R_{*} \\
0.035 R_{*} \\
0.035 R_{*} \\
0.035 R_{*} \\
0.035 R_{*} \\
0.044 R_{*} \\
0.052 R_{*} \\
0.052 R_{*} \\
0.052 R_{*} \\
0.061 R_{*} \\
0.070 R_{*}\end{array}$ & $\begin{array}{c}0.035 R_{*} \\
0.035 R_{*} \\
0.035 R_{*} \\
0.035 R_{*} \\
0.035 R_{*} \\
0.035 R_{*} \\
0.035 R_{*} \\
0.044 R_{*} \\
0.044 R_{*} \\
0.044 R_{*} \\
0.044 R_{*} \\
0.052 R_{*} \\
0.061 R_{*} \\
0.061 R_{*} \\
0.061 R_{*} \\
0.070 R_{*}\end{array}$ & $\begin{array}{c}0.035 R_{*} \\
0.035 R_{*} \\
0.035 R_{*} \\
0.035 R_{*} \\
0.035 R_{*} \\
0.035 R_{*} \\
0.035 R_{*} \\
0.044 R_{*} \\
0.044 R_{*} \\
0.044 R_{*} \\
0.044 R_{*} \\
0.052 R_{*} \\
0.061 R_{*} \\
0.061 R_{*} \\
0.061 R_{*} \\
0.070 R_{*}\end{array}$ & $\begin{array}{c}0.035 R_{*} \\
0.035 R_{*} \\
0.035 R_{*} \\
0.044 R_{*} \\
0.035 R_{*} \\
0.035 R_{*} \\
0.035 R_{*} \\
0.044 R_{*} \\
0.044 R_{*} \\
0.044 R_{*} \\
0.052 R_{*} \\
0.052 R_{*} \\
0.061 R_{*} \\
0.061 R_{*} \\
0.061 R_{*} \\
0.079 R_{*}\end{array}$ \\
\hline \multicolumn{11}{|c|}{$R_{\mathrm{p}}=2.25 R_{\oplus}$} \\
\hline $\begin{array}{l}3000 \\
3000 \\
3000 \\
3000 \\
3050 \\
3050 \\
3050 \\
3050 \\
3100 \\
3100 \\
3100 \\
3100 \\
3150 \\
3150 \\
3150 \\
3150\end{array}$ & $\begin{array}{c}60 \\
100 \\
150 \\
200 \\
60 \\
100 \\
150 \\
200 \\
60 \\
100 \\
150 \\
200 \\
60 \\
100 \\
150 \\
200\end{array}$ & $\begin{array}{c}0.026 R_{*} \\
0.026 R_{*} \\
0.026 R_{*} \\
0.035 R_{*} \\
0.035 R_{*} \\
0.035 R_{*} \\
0.035 R_{*} \\
0.035 R_{*} \\
0.044 R_{*} \\
0.044 R_{*} \\
0.044 R_{*} \\
0.044 R_{*} \\
0.052 R_{*} \\
0.052 R_{*} \\
0.052 R_{*} \\
0.061 R_{*}\end{array}$ & $\begin{array}{l}0.026 R_{*} \\
0.026 R_{*} \\
0.026 R_{*} \\
0.035 R_{*} \\
0.035 R_{*} \\
0.035 R_{*} \\
0.035 R_{*} \\
0.035 R_{*} \\
0.044 R_{*} \\
0.044 R_{*} \\
0.044 R_{*} \\
0.044 R_{*} \\
0.052 R_{*} \\
0.052 R_{*} \\
0.052 R_{*} \\
0.061 R_{*}\end{array}$ & $\begin{array}{c}0.026 R_{*} \\
0.026 R_{*} \\
0.026 R_{*} \\
0.035 R_{*} \\
0.035 R_{*} \\
0.035 R_{*} \\
0.035 R_{*} \\
0.035 R_{*} \\
0.044 R_{*} \\
0.044 R_{*} \\
0.044 R_{*} \\
0.044 R_{*} \\
0.052 R_{*} \\
0.052 R_{*} \\
0.052 R_{*} \\
0.061 R_{*}\end{array}$ & $\begin{array}{c}0.035 R_{*} \\
0.035 R_{*} \\
0.035 R_{*} \\
0.035 R_{*} \\
0.035 R_{*} \\
0.035 R_{*} \\
0.035 R_{*} \\
0.044 R_{*} \\
0.044 R_{*} \\
0.044 R_{*} \\
0.044 R_{*} \\
0.044 R_{*} \\
0.061 R_{*} \\
0.061 R_{*} \\
0.061 R_{*} \\
0.061 R_{*}\end{array}$ & $\begin{array}{c}0.035 R_{*} \\
0.035 R_{*} \\
0.035 R_{*} \\
0.035 R_{*} \\
0.035 R_{*} \\
0.035 R_{*} \\
0.035 R_{*} \\
0.044 R_{*} \\
0.044 R_{*} \\
0.044 R_{*} \\
0.044 R_{*} \\
0.044 R_{*} \\
0.061 R_{*} \\
0.061 R_{*} \\
0.061 R_{*} \\
0.070 R_{*}\end{array}$ & $\begin{array}{c}0.035 R_{*} \\
0.035 R_{*} \\
0.035 R_{*} \\
0.035 R_{*} \\
0.035 R_{*} \\
0.035 R_{*} \\
0.035 R_{*} \\
0.044 R_{*} \\
0.044 R_{*} \\
0.044 R_{*} \\
0.044 R_{*} \\
0.044 R_{*} \\
0.061 R_{*} \\
0.061 R_{*} \\
0.061 R_{*} \\
0.070 R_{*}\end{array}$ & $\begin{array}{c}0.035 R_{*} \\
0.035 R_{*} \\
0.035 R_{*} \\
0.035 R_{*} \\
0.044 R_{*} \\
0.044 R_{*} \\
0.044 R_{*} \\
0.044 R_{*} \\
0.044 R_{*} \\
0.044 R_{*} \\
0.044 R_{*} \\
0.052 R_{*} \\
0.061 R_{*} \\
0.061 R_{*} \\
0.061 R_{*} \\
0.070 R_{*}\end{array}$ & $\begin{array}{c}0.035 R_{*} \\
0.035 R_{*} \\
0.035 R_{*} \\
0.044 R_{*} \\
0.044 R_{*} \\
0.044 R_{*} \\
0.044 R_{*} \\
0.044 R_{*} \\
0.044 R_{*} \\
0.044 R_{*} \\
0.044 R_{*} \\
0.052 R_{*} \\
0.061 R_{*} \\
0.061 R_{*} \\
0.061 R_{*} \\
0.070 R_{*}\end{array}$ & $\begin{array}{c}0.035 R_{*} \\
0.035 R_{*} \\
0.035 R_{*} \\
0.044 R_{*} \\
0.044 R_{*} \\
0.044 R_{*} \\
0.044 R_{*} \\
0.044 R_{*} \\
0.044 R_{*} \\
0.044 R_{*} \\
0.044 R_{*} \\
0.052 R_{*} \\
0.070 R_{*} \\
0.070 R_{*} \\
0.070 R_{*} \\
0.079 R_{*}\end{array}$ \\
\hline
\end{tabular}


Table A.1. continued.

\begin{tabular}{|c|c|c|c|c|c|c|c|c|c|c|}
\hline \multirow{2}{*}{$\begin{array}{c}T_{\text {spot }} \\
(K)\end{array}$} & \multirow{2}{*}{$\begin{array}{l}\text { rms scatter } \\
\text { (ppm) }\end{array}$} & \multicolumn{3}{|c|}{$\begin{array}{c}\lambda_{\mathrm{obs}}=600 \mathrm{~nm} \\
P\end{array}$} & \multicolumn{3}{|c|}{$\begin{array}{c}\lambda_{\mathrm{obs}}=785 \mathrm{~nm} \\
P\end{array}$} & \multicolumn{3}{|c|}{$\begin{array}{c}\lambda_{\mathrm{obs}}=1000 \mathrm{~nm} \\
P\end{array}$} \\
\hline & & $1 \mathrm{~d}$ & $2 d$ & $3 d$ & $1 \mathrm{~d}$ & $2 \mathrm{~d}$ & $3 d$ & $1 \mathrm{~d}$ & $2 d$ & $3 d$ \\
\hline \multicolumn{11}{|c|}{$R_{\mathrm{p}}=2.50 R_{\oplus}$} \\
\hline 3000 & 60 & $0.035 R_{*}$ & $0.035 R_{*}$ & $0.035 R_{*}$ & $0.035 R_{*}$ & $0.035 R_{*}$ & $0.035 R_{*}$ & $0.044 R_{*}$ & $0.044 R_{*}$ & $0.044 R_{*}$ \\
\hline 3000 & 100 & $0.035 R_{*}$ & $0.035 R_{*}$ & $0.035 R_{*}$ & $0.035 R_{*}$ & $0.035 R_{*}$ & $0.035 R_{*}$ & $0.044 R_{*}$ & $0.044 R_{*}$ & $0.044 R_{*}$ \\
\hline 3000 & 150 & $0.035 R_{*}$ & $0.035 R_{*}$ & $0.035 R_{*}$ & $0.035 R_{*}$ & $0.035 R_{*}$ & $0.035 R_{*}$ & $0.044 R_{*}$ & $0.044 R_{*}$ & $0.044 R_{*}$ \\
\hline 3000 & 200 & $0.035 R_{*}$ & $0.035 R_{*}$ & $0.035 R_{*}$ & $0.035 R_{*}$ & $0.035 R_{*}$ & $0.035 R_{*}$ & $0.044 R_{*}$ & $0.044 R_{*}$ & $0.044 R_{*}$ \\
\hline 3050 & 60 & $0.035 R_{*}$ & $0.035 R_{*}$ & $0.035 R_{*}$ & $0.044 R_{*}$ & $0.044 R_{*}$ & $0.044 R_{*}$ & $0.044 R_{*}$ & $0.044 R_{*}$ & $0.044 R_{*}$ \\
\hline 3050 & 100 & $0.035 R_{*}$ & $0.035 R_{*}$ & $0.035 R_{*}$ & $0.044 R_{*}$ & $0.044 R_{*}$ & $0.044 R_{*}$ & $0.044 R_{*}$ & $0.044 R_{*}$ & $0.044 R_{*}$ \\
\hline 3050 & 150 & $0.035 R_{*}$ & $0.035 R_{*}$ & $0.035 R_{*}$ & $0.044 R_{*}^{*}$ & $0.044 R_{*}^{*}$ & $0.044 R_{*}^{*}$ & $0.044 R_{*}^{*}$ & $0.044 R_{*}$ & $0.044 R_{*}$ \\
\hline 3050 & 200 & $0.035 R_{*}$ & $0.035 R_{*}$ & $0.035 R_{*}$ & $0.044 R_{*}$ & $0.044 R_{*}$ & $0.044 R_{*}$ & $0.044 R_{*}$ & $0.044 R_{*}$ & $0.044 R_{*}$ \\
\hline 3100 & 60 & $0.044 R_{*}$ & $0.044 R_{*}$ & $0.044 R_{*}$ & $0.044 R_{*}$ & $0.044 R_{*}$ & $0.044 R_{*}$ & $0.052 R_{*}$ & $0.052 R_{*}$ & $0.052 R_{*}$ \\
\hline 3100 & 100 & $0.044 R_{*}$ & $0.044 R_{*}$ & $0.044 R_{*}$ & $0.044 R_{*}$ & $0.044 R_{*}$ & $0.044 R_{*}$ & $0.052 R_{*}$ & $0.052 R_{*}$ & $0.052 R_{*}$ \\
\hline 3100 & 150 & $0.044 R_{*}$ & $0.044 R_{*}$ & $0.044 R_{*}$ & $0.044 R_{*}$ & $0.044 R_{*}$ & $0.044 R_{*}$ & $0.052 R_{*}$ & $0.052 R_{*}$ & $0.052 R_{*}$ \\
\hline 3100 & 200 & $0.044 R_{*}$ & $0.044 R_{*}$ & $0.044 R_{*}$ & $0.044 R_{*}$ & $0.044 R_{*}$ & $0.044 R_{*}$ & $0.052 R_{*}$ & $0.052 R_{*}$ & $0.052 R_{*}$ \\
\hline 3150 & 60 & $0.052 R_{*}$ & $0.052 R_{*}$ & $0.052 R_{*}$ & $0.061 R_{*}$ & $0.061 R_{*}$ & $0.061 R_{*}$ & $0.070 R_{*}$ & $0.070 R_{*}$ & $0.070 R_{*}$ \\
\hline 3150 & 100 & $0.052 R_{*}$ & $0.052 R_{*}$ & $0.052 R_{*}$ & $0.061 R_{*}$ & $0.061 R_{*}$ & $0.061 R_{*}$ & $0.070 R_{*}$ & $0.070 R_{*}$ & $0.070 R_{*}$ \\
\hline 3150 & 150 & $0.052 R_{*}$ & $0.052 R_{*}$ & $0.052 R_{*}$ & $0.061 R_{*}$ & $0.061 R_{*}$ & $0.061 R_{*}$ & $0.070 R_{*}$ & $0.070 R_{*}$ & $0.070 R_{*}$ \\
\hline 3150 & 200 & $0.052 R_{*}$ & $0.052 R_{*}$ & $0.061 R_{*}$ & $0.061 R_{*}$ & $0.061 R_{*}$ & $0.061 R_{*}$ & $0.070 R_{*}$ & $0.070 R_{*}$ & $0.070 R_{*}$ \\
\hline \multicolumn{11}{|c|}{$R_{\mathrm{p}}=2.75 R_{\oplus}$} \\
\hline 3000 & 60 & $0.035 R_{*}$ & $0.035 R_{*}$ & $0.035 R_{*}$ & $0.035 R_{*}$ & $0.035 R_{*}$ & $0.035 R_{*}$ & $0.044 R_{*}$ & $0.044 R_{*}$ & $0.044 R_{*}$ \\
\hline 3000 & 100 & $0.035 R_{*}$ & $0.035 R_{*}$ & $0.035 R_{*}$ & $0.035 R_{*}$ & $0.035 R_{*}$ & $0.035 R_{*}$ & $0.044 R_{*}$ & $0.044 R_{*}$ & $0.044 R_{*}$ \\
\hline 3000 & 150 & $0.035 R_{*}$ & $0.035 R_{*}$ & $0.035 R_{*}$ & $0.035 R_{*}$ & $0.035 R_{*}$ & $0.035 R_{*}$ & $0.044 R_{*}$ & $0.044 R_{*}$ & $0.044 R_{*}$ \\
\hline 3000 & 200 & $0.035 R_{*}$ & $0.035 R_{*}$ & $0.035 R_{*}$ & $0.035 R_{*}$ & $0.035 R_{*}$ & $0.035 R_{*}$ & $0.044 R_{*}$ & $0.044 R_{*}$ & $0.044 R_{*}$ \\
\hline 3050 & 60 & $0.035 R_{*}$ & $0.035 R_{*}$ & $0.035 R_{*}$ & $0.044 R_{*}$ & $0.044 R_{*}$ & $0.044 R_{*}$ & $0.044 R_{*}$ & $0.044 R_{*}$ & $0.044 R_{*}$ \\
\hline 3050 & 100 & $0.035 R_{*}$ & $0.035 R_{*}$ & $0.035 R_{*}$ & $0.044 R_{*}$ & $0.044 R_{*}$ & $0.044 R_{*}$ & $0.044 R_{*}$ & $0.044 R_{*}$ & $0.044 R_{*}$ \\
\hline 3050 & 150 & $0.035 R_{*}$ & $0.035 R_{*}$ & $0.035 R_{*}$ & $0.044 R_{*}$ & $0.044 R_{*}$ & $0.044 R_{*}$ & $0.044 R_{*}$ & $0.044 R_{*}$ & $0.044 R_{*}$ \\
\hline 3050 & 200 & $0.035 R_{*}$ & $0.035 R_{*}$ & $0.035 R_{*}$ & $0.044 R_{*}$ & $0.044 R_{*}$ & $0.044 R_{*}$ & $0.044 R_{*}$ & $0.044 R_{*}$ & $0.044 R_{*}$ \\
\hline 3100 & 60 & $0.044 R_{*}$ & $0.044 R_{*}$ & $0.044 R_{*}$ & $0.052 R_{*}$ & $0.052 R_{*}$ & $0.052 R_{*}$ & $0.052 R_{*}$ & $0.052 R_{*}$ & $0.052 R_{*}$ \\
\hline 3100 & 100 & $0.044 R_{*}$ & $0.044 R_{*}$ & $0.044 R_{*}$ & $0.052 R_{*}$ & $0.052 R_{*}$ & $0.052 R_{*}$ & $0.052 R_{*}$ & $0.052 R_{*}$ & $0.052 R_{*}$ \\
\hline 3100 & 150 & $0.044 R_{*}$ & $0.044 R_{*}$ & $0.044 R_{*}$ & $0.052 R_{*}$ & $0.052 R_{*}$ & $0.052 R_{*}$ & $0.052 R_{*}$ & $0.052 R_{*}$ & $0.052 R_{*}$ \\
\hline 3100 & 200 & $0.044 R_{*}$ & $0.044 R_{*}$ & $0.044 R_{*}$ & $0.052 R_{*}$ & $0.052 R_{*}$ & $0.052 R_{*}$ & $0.052 R_{*}$ & $0.052 R_{*}$ & $0.052 R_{*}$ \\
\hline 3150 & 60 & $0.061 R_{*}^{*}$ & $0.061 R_{*}^{*}$ & $0.061 R_{*}^{*}$ & $0.070 R_{*}^{*}$ & $0.070 R_{*}^{*}$ & $0.070 R_{*}^{*}$ & $0.079 R_{*}^{*}$ & $0.079 R_{*}^{*}$ & $0.079 R_{*}$ \\
\hline 3150 & 100 & $0.061 R_{*}^{*}$ & $0.061 R_{*}^{*}$ & $0.061 R_{*}^{*}$ & $0.070 R_{*}^{*}$ & $0.070 R_{*}^{*}$ & $0.070 R_{*}^{*}$ & $0.079 R_{*}^{*}$ & $0.079 R_{*}^{*}$ & $0.079 R_{*}$ \\
\hline 3150 & 150 & $0.061 R_{*}$ & $0.061 R_{*}$ & $0.061 R_{*}$ & $0.070 R_{*}$ & $0.070 R_{*}$ & $0.070 R_{*}$ & $0.079 R_{*}$ & $0.079 R_{*}$ & $0.079 R_{*}$ \\
\hline 3150 & 200 & $0.061 R_{*}$ & $0.061 R_{*}$ & $0.061 R_{*}$ & $0.070 R_{*}$ & $0.070 R_{*}$ & $0.070 R_{*}$ & $0.079 R_{*}$ & $0.079 R_{*}$ & $0.079 R_{*}$ \\
\hline \multicolumn{11}{|c|}{$R_{\mathrm{p}}=3.00 R_{\oplus}$} \\
\hline 3000 & 60 & $0.035 R_{*}$ & $0.035 R_{*}$ & $0.035 R_{*}$ & $0.044 R_{*}$ & $0.044 R_{*}$ & $0.044 R_{*}$ & $0.044 R_{*}$ & $0.044 R_{*}$ & $0.044 R_{*}$ \\
\hline 3000 & 100 & $0.035 R_{*}$ & $0.035 R_{*}$ & $0.035 R_{*}^{*}$ & $0.044 R_{*}$ & $0.044 R_{*}$ & $0.044 R_{*}$ & $0.044 R_{*}$ & $0.044 R_{*}$ & $0.044 R_{*}$ \\
\hline 3000 & 150 & $0.035 R_{*}$ & $0.035 R_{*}$ & $0.035 R_{*}$ & $0.044 R_{*}$ & $0.044 R_{*}$ & $0.044 R_{*}$ & $0.044 R_{*}$ & $0.044 R_{*}$ & $0.044 R_{*}$ \\
\hline 3000 & 200 & $0.035 R_{*}$ & $0.035 R_{*}$ & $0.035 R_{*}$ & $0.044 R_{*}$ & $0.044 R_{*}$ & $0.044 R_{*}$ & $0.044 R_{*}$ & $0.044 R_{*}$ & $0.044 R_{*}$ \\
\hline 3050 & 60 & $0.044 R_{*}$ & $0.044 R_{*}$ & $0.044 R_{*}$ & $0.044 R_{*}$ & $0.044 R_{*}$ & $0.044 R_{*}$ & $0.052 R_{*}$ & $0.052 R_{*}$ & $0.052 R_{*}$ \\
\hline 3050 & 100 & $0.044 R_{*}$ & $0.044 R_{*}$ & $0.044 R_{*}$ & $0.044 R_{*}$ & $0.044 R_{*}$ & $0.044 R_{*}$ & $0.052 R_{*}$ & $0.052 R_{*}$ & $0.052 R_{*}$ \\
\hline 3050 & 150 & $0.044 R_{*}$ & $0.044 R_{*}$ & $0.044 R_{*}$ & $0.044 R_{*}$ & $0.044 R_{*}$ & $0.044 R_{*}$ & $0.052 R_{*}$ & $0.052 R_{*}$ & $0.052 R_{*}$ \\
\hline 3050 & 200 & $0.044 R_{*}$ & $0.044 R_{*}$ & $0.044 R_{*}$ & $0.044 R_{*}$ & $0.044 R_{*}$ & $0.044 R_{*}$ & $0.052 R_{*}$ & $0.052 R_{*}$ & $0.052 R_{*}$ \\
\hline 3100 & 60 & $0.052 R_{*}$ & $0.052 R_{*}$ & $0.052 R_{*}$ & $0.052 R_{*}$ & $0.052 R_{*}$ & $0.052 R_{*}$ & $0.061 R_{*}$ & $0.061 R_{*}$ & $0.061 R_{*}$ \\
\hline 3100 & 100 & $0.052 R_{*}$ & $0.052 R_{*}$ & $0.052 R_{*}$ & $0.052 R_{*}$ & $0.052 R_{*}$ & $0.052 R_{*}$ & $0.061 R_{*}$ & $0.061 R_{*}$ & $0.061 R_{*}$ \\
\hline 3100 & 150 & $0.052 R_{*}$ & $0.052 R_{*}$ & $0.052 R_{*}$ & $0.052 R_{*}$ & $0.052 R_{*}$ & $0.052 R_{*}$ & $0.061 R_{*}$ & $0.061 R_{*}$ & $0.061 R_{*}$ \\
\hline 3100 & 200 & $0.052 R_{*}$ & $0.052 R_{*}$ & $0.052 R_{*}$ & $0.052 R_{*}$ & $0.052 R_{*}$ & $0.052 R_{*}$ & $0.061 R_{*}$ & $0.061 R_{*}$ & $0.061 R_{*}$ \\
\hline 3150 & 60 & $0.070 R_{*}$ & $0.070 R_{*}$ & $0.070 R_{*}$ & $0.079 R_{*}$ & $0.079 R_{*}$ & $0.079 R_{*}$ & $0.087 R_{*}$ & $0.087 R_{*}$ & $0.087 R_{*}$ \\
\hline 3150 & 100 & $0.070 R_{*}$ & $0.070 R_{*}$ & $0.070 R_{*}$ & $0.079 R_{*}$ & $0.079 R_{*}$ & $0.079 R_{*}$ & $0.087 R_{*}$ & $0.087 R_{*}$ & $0.087 R_{*}$ \\
\hline 3150 & 150 & $0.070 R_{*}$ & $0.070 R_{*}$ & $0.070 R_{*}$ & $0.079 R_{*}$ & $0.079 R_{*}$ & $0.079 R_{*}$ & $0.087 R_{*}$ & $0.087 R_{*}$ & $0.087 R_{*}$ \\
\hline 3150 & 200 & $0.070 R_{*}$ & $0.070 R_{*}$ & $0.070 R_{*}$ & $0.079 R_{*}$ & $0.079 R_{*}$ & $0.079 R_{*}$ & $0.087 R_{*}$ & $0.087 R_{*}$ & $0.087 R_{*}$ \\
\hline
\end{tabular}


A\&A 630, A114 (2019)

Table A.2. Smallest detectable value of $r_{\text {spot }}$ determined from the 7812 simulations of the 1296 scenarios that used an M1V dwarf host star.

\begin{tabular}{|c|c|c|c|c|c|c|c|c|c|c|}
\hline \multirow{2}{*}{$\begin{array}{l}T_{\text {spot }} \\
(K)\end{array}$} & \multirow{2}{*}{$\begin{array}{l}\text { rms scatter } \\
\text { (ppm) }\end{array}$} & \multicolumn{3}{|c|}{$\begin{array}{c}\lambda_{\mathrm{obs}}=600 \mathrm{~nm} \\
P\end{array}$} & \multicolumn{3}{|c|}{$\begin{array}{c}\lambda_{\mathrm{obs}}=785 \mathrm{~nm} \\
P\end{array}$} & \multicolumn{3}{|c|}{$\begin{array}{c}\lambda_{\mathrm{obs}}=1000 \mathrm{~nm} \\
P\end{array}$} \\
\hline & & $2 \mathrm{~d}$ & $3 \mathrm{~d}$ & $4 \mathrm{~d}$ & $2 \mathrm{~d}$ & $3 \mathrm{~d}$ & $4 \mathrm{~d}$ & $2 \mathrm{~d}$ & $3 \mathrm{~d}$ & $4 \mathrm{~d}$ \\
\hline \multicolumn{11}{|c|}{$R_{\mathrm{p}}=2.00 R_{\oplus}$} \\
\hline 3400 & 60 & $0.017 R_{*}\left(0.056 R_{*}\right)$ & $0.026 R_{*}\left(0.083 R_{*}\right)$ & $0.026 R_{*}\left(0.083 R_{*}\right)$ & $0.017 R_{*}\left(0.056 R_{*}\right)$ & $0.026 R_{*}\left(0.083 R_{*}\right)$ & $0.026 R_{*}\left(0.083 R_{*}\right)$ & $0.026 R_{*}\left(0.083 R_{*}\right)$ & $0.026 R_{*}\left(0.083 R_{*}\right)$ & $0.026 R_{*}\left(0.083 R_{*}\right)$ \\
\hline 3400 & 100 & $0.026 R_{*}\left(0.083 R_{*}\right)$ & $0.026 R_{*}\left(0.083 R_{*}\right)$ & $0.035 R_{*}\left(0.111 R_{*}\right)$ & $0.026 R_{*}\left(0.083 R_{*}\right)$ & $0.035 R_{*}\left(0.111 R_{*}\right)$ & $0.035 R_{*}\left(0.111 R_{*}\right)$ & $0.035 R_{*}\left(0.111 R_{*}\right)$ & $0.035 R_{*}\left(0.111 R_{*}\right)$ & $0.044 R_{*}\left(0.139 R_{*}\right)$ \\
\hline 3400 & 150 & $0.035 R_{*}\left(0.111 R_{*}\right)$ & $0.035 R_{*}\left(0.111 R_{*}\right)$ & $0.044 R_{*}\left(0.139 R_{*}\right)$ & $0.035 R_{*}\left(0.111 R_{*}\right)$ & $0.044 R_{*}\left(0.139 R_{*}\right)$ & $0.044 R_{*}\left(0.139 R_{*}\right)$ & $0.044 R_{*}\left(0.139 R_{*}\right)$ & $0.044 R_{*}\left(0.139 R_{*}\right)$ & $0.052 R_{*}\left(0.167 R_{*}\right)$ \\
\hline 3400 & 200 & $0.035 R_{*}\left(0.111 R_{*}\right)$ & $0.044 R_{*}\left(0.139 R_{*}\right)$ & $0.044 R_{*}\left(0.139 R_{*}\right)$ & $0.044 R_{*}\left(0.139 R_{*}\right)$ & $0.044 R_{*}\left(0.139 R_{*}\right)$ & $0.052 R_{*}\left(0.167 R_{*}\right)$ & $0.044 R_{*}\left(0.139 R_{*}\right)$ & $0.052 R_{*}\left(0.167 R_{*}\right)$ & $0.061 R_{*}\left(0.194 R_{*}\right)$ \\
\hline 3475 & 60 & $0.017 R_{*}\left(0.056 R_{*}\right)$ & $0.026 R_{*}\left(0.083 R_{*}\right)$ & $0.026 R_{*}\left(0.083 R_{*}\right)$ & $0.026 R_{*}\left(0.083 R_{*}\right)$ & $0.026 R_{*}\left(0.083 R_{*}\right)$ & $0.026 R_{*}\left(0.083 R_{*}\right)$ & $0.026 R_{*}\left(0.083 R_{*}\right)$ & $0.026 R_{*}\left(0.083 R_{*}\right)$ & $0.035 R_{*}\left(0.111 R_{*}\right)$ \\
\hline 3475 & 100 & $0.026 R_{*}\left(0.083 R_{*}\right)$ & $0.035 R_{*}\left(0.111 R_{*}\right)$ & $0.035 R_{*}\left(0.111 R_{*}\right)$ & $0.035 R_{*}\left(0.111 R_{*}\right)$ & $0.035 R_{*}\left(0.111 R_{*}\right)$ & $0.044 R_{*}\left(0.139 R_{*}\right)$ & $0.035 R_{*}\left(0.111 R_{*}\right)$ & $0.035 R_{*}\left(0.111 R_{*}\right)$ & $0.044 R_{*}\left(0.139 R_{*}\right)$ \\
\hline 3475 & 150 & $0.035 R_{*}\left(0.111 R_{*}\right)$ & $0.044 R_{*}\left(0.139 R_{*}\right)$ & $0.044 R_{*}\left(0.139 R_{*}\right)$ & $0.044 R_{*}\left(0.139 R_{*}\right)$ & $0.044 R_{*}\left(0.139 R_{*}\right)$ & $0.052 R_{*}\left(0.167 R_{*}\right)$ & $0.052 R_{*}\left(0.167 R_{*}\right)$ & $0.052 R_{*}\left(0.167 R_{*}\right)$ & $0.061 R_{*}\left(0.194 R_{*}\right)$ \\
\hline 3475 & 200 & $0.044 R_{*}\left(0.139 R_{*}\right)$ & $0.052 R_{*}\left(0.167 R_{*}\right)$ & $0.052 R_{*}\left(0.167 R_{*}\right)$ & $0.052 R_{*}\left(0.167 R_{*}\right)$ & $0.052 R_{*}\left(0.167 R_{*}\right)$ & $0.061 R_{*}\left(0.194 R_{*}\right)$ & $0.061 R_{*}\left(0.194 R_{*}\right)$ & $0.061 R_{*}\left(0.194 R_{*}\right)$ & $0.079 R_{*}\left(0.250 R_{*}\right)$ \\
\hline 3550 & 60 & $0.026 R_{*}\left(0.083 R_{*}\right)$ & $0.026 R_{*}\left(0.083 R_{*}\right)$ & $0.026 R_{*}\left(0.083 R_{*}\right)$ & $0.026 R_{*}\left(0.083 R_{*}\right)$ & $0.026 R_{*}\left(0.083 R_{*}\right)$ & $0.035 R_{*}\left(0.111 R_{*}\right)$ & $0.035 R_{*}\left(0.111 R_{*}\right)$ & $0.035 R_{*}\left(0.111 R_{*}\right)$ & $0.035 R_{*}\left(0.111 R_{*}\right)$ \\
\hline 3550 & 100 & $0.035 R_{*}\left(0.111 R_{*}\right)$ & $0.035 R_{*}\left(0.111 R_{*}\right)$ & $0.044 R_{*}\left(0.139 R_{*}\right)$ & $0.035 R_{*}\left(0.111 R_{*}\right)$ & $0.044 R_{*}\left(0.139 R_{*}\right)$ & $0.052 R_{*}\left(0.167 R_{*}\right)$ & $0.044 R_{*}\left(0.139 R_{*}\right)$ & $0.052 R_{*}\left(0.167 R_{*}\right)$ & $0.061 R_{*}\left(0.194 R_{*}\right)$ \\
\hline 3550 & 150 & $0.044 R_{*}\left(0.139 R_{*}\right)$ & $0.052 R_{*}\left(0.167 R_{*}\right)$ & $0.061 R_{*}\left(0.194 R_{*}\right)$ & $0.052 R_{*}\left(0.167 R_{*}\right)$ & $0.061 R_{*}\left(0.194 R_{*}\right)$ & $0.070 R_{*}\left(0.222 R_{*}\right)$ & $0.070 R_{*}\left(0.222 R_{*}\right)$ & $0.087 R_{*}\left(0.278 R_{*}\right)$ & $0.087 R_{*}\left(0.278 R_{*}\right)$ \\
\hline 3550 & 200 & $0.052 R_{*}\left(0.167 R_{*}\right)$ & $0.061 R_{*}\left(0.194 R_{*}\right)$ & $0.070 R_{*}\left(0.222 R_{*}\right)$ & $0.079 R_{*}\left(0.250 R_{*}\right)$ & $0.096 R_{*}\left(0.305 R_{*}\right)$ & $0.105 R_{*}\left(0.333 R_{*}\right)$ & $\mathrm{NaN}$ & $\mathrm{NaN}$ & $\mathrm{NaN}$ \\
\hline 3625 & 60 & $0.035 R_{*}\left(0.111 R_{*}\right)$ & $0.044 R_{*}\left(0.139 R_{*}\right)$ & $0.044 R_{*}\left(0.139 R_{*}\right)$ & $0.044 R_{*}\left(0.139 R_{*}\right)$ & $0.044 R_{*}\left(0.139 R_{*}\right)$ & $0.052 R_{*}\left(0.167 R_{*}\right)$ & $0.044 R_{*}\left(0.139 R_{*}\right)$ & $0.052 R_{*}\left(0.167 R_{*}\right)$ & $0.061 R_{*}\left(0.194 R_{*}\right)$ \\
\hline 3625 & 100 & $0.052 R_{*}\left(0.167 R_{*}\right)$ & $0.061 R_{*}\left(0.194 R_{*}\right)$ & $0.070 R_{*}\left(0.222 R_{*}\right)$ & $0.087 R_{*}\left(0.278 R_{*}\right)$ & $0.105 R_{*}\left(0.333 R_{*}\right)$ & $0.096 R_{*}\left(0.305 R_{*}\right)$ & $\mathrm{NaN}$ & $\mathrm{NaN}$ & $\mathrm{NaN}$ \\
\hline 3625 & 150 & $\mathrm{NaN}$ & $\mathrm{NaN}$ & $\mathrm{NaN}$ & $\mathrm{NaN}$ & $\mathrm{NaN}$ & $\mathrm{NaN}$ & $\mathrm{NaN}$ & $\mathrm{NaN}$ & $\mathrm{NaN}$ \\
\hline 3625 & 200 & $\mathrm{NaN}$ & $\mathrm{NaN}$ & $\mathrm{NaN}$ & $\mathrm{NaN}$ & $\mathrm{NaN}$ & $\mathrm{NaN}$ & $\mathrm{NaN}$ & $\mathrm{NaN}$ & $\mathrm{NaN}$ \\
\hline
\end{tabular}

$R_{\mathrm{p}}=2.25 R_{\oplus}$

\begin{tabular}{|c|c|c|c|c|c|c|c|c|c|c|}
\hline 3400 & 60 & $0.017 R_{*}\left(0.056 R_{*}\right)$ & $0.017 R_{*}\left(0.056 R_{*}\right)$ & $0.026 R_{*}\left(0.083 R_{*}\right)$ & $0.017 R_{*}\left(0.056 R_{*}\right)$ & $0.026 R_{*}\left(0.083 R_{*}\right)$ & $0.026 R_{*}\left(0.083 R_{*}\right)$ & $0.026 R_{*}\left(0.083 R_{*}\right)$ & $0.026 R_{*}\left(0.083 R_{*}\right)$ & $0.026 R_{*}\left(0.083 R_{*}\right)$ \\
\hline 3400 & 100 & $0.026 R_{*}\left(0.083 R_{*}\right)$ & $0.026 R_{*}\left(0.083 R_{*}\right)$ & $0.035 R_{*}\left(0.111 R_{*}\right)$ & $0.026 R_{*}\left(0.083 R_{*}\right)$ & $0.035 R_{*}\left(0.111 R_{*}\right)$ & $0.035 R_{*}\left(0.111 R_{*}\right)$ & $0.026 R_{*}\left(0.083 R_{*}\right)$ & $0.035 R_{*}\left(0.111 R_{*}\right)$ & $0.035 R_{*}\left(0.111 R_{*}\right)$ \\
\hline 3400 & 150 & $0.035 R_{*}\left(0.111 R_{*}\right)$ & $0.035 R_{*}\left(0.111 R_{*}\right)$ & $0.035 R_{*}\left(0.111 R_{*}\right)$ & $0.035 R_{*}\left(0.111 R_{*}\right)$ & $0.035 R_{*}\left(0.111 R_{*}\right)$ & $0.044 R_{*}\left(0.139 R_{*}\right)$ & $0.035 R_{*}\left(0.111 R_{*}\right)$ & $0.044 R_{*}\left(0.139 R_{*}\right)$ & $0.044 R_{*}\left(0.139 R_{*}\right)$ \\
\hline 3400 & 200 & $0.035 R_{*}\left(0.111 R_{*}\right)$ & $0.035 R_{*}\left(0.111 R_{*}\right)$ & $0.044 R_{*}\left(0.139 R_{*}\right)$ & $0.044 R_{*}\left(0.139 R_{*}\right)$ & $0.044 R_{*}\left(0.139 R_{*}\right)$ & $0.044 R_{*}\left(0.139 R_{*}\right)$ & $0.044 R_{*}\left(0.139 R_{*}\right)$ & $0.044 R_{*}\left(0.139 R_{*}\right)$ & $0.052 R_{*}\left(0.167 R_{*}\right)$ \\
\hline 3475 & 60 & $0.017 R_{*}\left(0.056 R_{*}\right)$ & $0.026 R_{*}\left(0.083 R_{*}\right)$ & $0.026 R_{*}\left(0.083 R_{*}\right)$ & $0.026 R_{*}\left(0.083 R_{*}\right)$ & $0.026 R_{*}\left(0.083 R_{*}\right)$ & $0.026 R_{*}\left(0.083 R_{*}\right)$ & $0.026 R_{*}\left(0.083 R_{*}\right)$ & $0.026 R_{*}\left(0.083 R_{*}\right)$ & $0.026 R_{*}\left(0.083 R_{*}\right)$ \\
\hline 3475 & 100 & $0.026 R_{*}\left(0.083 R_{*}\right)$ & $0.035 R_{*}\left(0.111 R_{*}\right)$ & $0.035 R_{*}\left(0.111 R_{*}\right)$ & $0.035 R_{*}\left(0.111 R_{*}\right)$ & $0.035 R_{*}\left(0.111 R_{*}\right)$ & $0.035 R_{*}\left(0.111 R_{*}\right)$ & $0.035 R_{*}\left(0.111 R_{*}\right)$ & $0.035 R_{*}\left(0.111 R_{*}\right)$ & $0.044 R_{*}\left(0.139 R_{*}\right)$ \\
\hline 3475 & 150 & $0.035 R_{*}\left(0.111 R_{*}\right)$ & $0.035 R_{*}\left(0.111 R_{*}\right)$ & $0.044 R_{*}\left(0.139 R_{*}\right)$ & $0.035 R_{*}\left(0.111 R_{*}\right)$ & $0.044 R_{*}\left(0.139 R_{*}\right)$ & $0.044 R_{*}\left(0.139 R_{*}\right)$ & $0.044 R_{*}\left(0.139 R_{*}\right)$ & $0.044 R_{*}\left(0.139 R_{*}\right)$ & $0.052 R_{*}\left(0.167 R_{*}\right)$ \\
\hline 3475 & 200 & $0.044 R_{*}\left(0.139 R_{*}\right)$ & $0.044 R_{*}\left(0.139 R_{*}\right)$ & $0.044 R_{*}\left(0.139 R_{*}\right)$ & $0.044 R_{*}\left(0.139 R_{*}\right)$ & $0.052 R_{*}\left(0.167 R_{*}\right)$ & $0.052 R_{*}\left(0.167 R_{*}\right)$ & $0.052 R_{*}\left(0.167 R_{*}\right)$ & $0.061 R_{*}\left(0.194 R_{*}\right)$ & $0.061 R_{*}\left(0.194 R_{*}\right)$ \\
\hline 3550 & 60 & $0.026 R_{*}\left(0.083 R_{*}\right)$ & $0.026 R_{*}\left(0.083 R_{*}\right)$ & $0.026 R_{*}\left(0.083 R_{*}\right)$ & $0.026 R_{*}\left(0.083 R_{*}\right)$ & $0.026 R_{*}\left(0.083 R_{*}\right)$ & $0.035 R_{*}\left(0.111 R_{*}\right)$ & $0.026 R_{*}\left(0.083 R_{*}\right)$ & $0.035 R_{*}\left(0.111 R_{*}\right)$ & $0.035 R_{*}\left(0.111 R_{*}\right)$ \\
\hline 3550 & 100 & $0.035 R_{*}\left(0.111 R_{*}\right)$ & $0.035 R_{*}\left(0.111 R_{*}\right)$ & $0.044 R_{*}\left(0.139 R_{*}\right)$ & $0.035 R_{*}\left(0.111 R_{*}\right)$ & $0.044 R_{*}\left(0.139 R_{*}\right)$ & $0.044 R_{*}\left(0.139 R_{*}\right)$ & $0.044 R_{*}\left(0.139 R_{*}\right)$ & $0.044 R_{*}\left(0.139 R_{*}\right)$ & $0.052 R_{*}\left(0.167 R_{*}\right)$ \\
\hline 3550 & 150 & $0.044 R_{*}\left(0.139 R_{*}\right)$ & $0.044 R_{*}\left(0.139 R_{*}\right)$ & $0.052 R_{*}\left(0.167 R_{*}\right)$ & $0.044 R_{*}\left(0.139 R_{*}\right)$ & $0.052 R_{*}\left(0.167 R_{*}\right)$ & $0.061 R_{*}\left(0.194 R_{*}\right)$ & $0.061 R_{*}\left(0.194 R_{*}\right)$ & $0.061 R_{*}\left(0.194 R_{*}\right)$ & $0.070 R_{*}\left(0.222 R_{*}\right)$ \\
\hline 3550 & 200 & $0.052 R_{*}\left(0.167 R_{*}\right)$ & $0.052 R_{*}\left(0.167 R_{*}\right)$ & $0.061 R_{*}\left(0.194 R_{*}\right)$ & $0.061 R_{*}\left(0.194 R_{*}\right)$ & $0.070 R_{*}\left(0.222 R_{*}\right)$ & $0.079 R_{*}\left(0.250 R_{*}\right)$ & $0.087 R_{*}\left(0.278 R_{*}\right)$ & $0.087 R_{*}\left(0.278 R_{*}\right)$ & $0.096 R_{*}\left(0.305 R_{*}\right)$ \\
\hline 3625 & 60 & $0.035 R_{*}\left(0.111 R_{*}\right)$ & $0.035 R_{*}\left(0.111 R_{*}\right)$ & $0.044 R_{*}\left(0.139 R_{*}\right)$ & $0.035 R_{*}\left(0.111 R_{*}\right)$ & $0.044 R_{*}\left(0.139 R_{*}\right)$ & $0.044 R_{*}\left(0.139 R_{*}\right)$ & $0.044 R_{*}\left(0.139 R_{*}\right)$ & $0.044 R_{*}\left(0.139 R_{*}\right)$ & $0.052 R_{*}\left(0.167 R_{*}\right)$ \\
\hline 3625 & 100 & $0.044 R_{*}\left(0.139 R_{*}\right)$ & $0.052 R_{*}\left(0.167 R_{*}\right)$ & $0.061 R_{*}\left(0.194 R_{*}\right)$ & $0.061 R_{*}\left(0.194 R_{*}\right)$ & $0.061 R_{*}\left(0.194 R_{*}\right)$ & $0.070 R_{*}\left(0.222 R_{*}\right)$ & $0.070 R_{*}\left(0.222 R_{*}\right)$ & $0.087 R_{*}\left(0.278 R_{*}\right)$ & $0.096 R_{*}\left(0.305 R_{*}\right)$ \\
\hline 3625 & 150 & $0.079 R_{*}\left(0.250 R_{*}\right)$ & $0.070 R_{*}\left(0.222 R_{*}\right)$ & $0.096 R_{*}\left(0.305 R_{*}\right)$ & $\mathrm{NaN}$ & $\mathrm{NaN}$ & $\mathrm{NaN}$ & $\mathrm{NaN}$ & $\mathrm{NaN}$ & $\mathrm{NaN}$ \\
\hline 3625 & 200 & $\mathrm{NaN}$ & $\mathrm{NaN}$ & $\mathrm{NaN}$ & $\mathrm{NaN}$ & $\mathrm{NaN}$ & $\mathrm{NaN}$ & $\mathrm{NaN}$ & $\mathrm{NaN}$ & $\mathrm{NaN}$ \\
\hline
\end{tabular}

$R_{\mathrm{p}}=2.50 R_{\oplus}$

\begin{tabular}{|c|c|c|c|c|c|c|c|c|c|c|}
\hline 3400 & 60 & $0.017 R_{*}\left(0.056 R_{*}\right)$ & $0.017 R_{*}\left(0.056 R_{*}\right)$ & $0.017 R_{*}\left(0.056 R_{*}\right)$ & $0.017 R_{*}\left(0.056 R_{*}\right)$ & $0.017 R_{*}\left(0.056 R_{*}\right)$ & $0.026 R_{*}\left(0.083 R_{*}\right)$ & $0.017 R_{*}\left(0.056 R_{*}\right)$ & $0.026 R_{*}\left(0.083 R_{*}\right)$ & $0.026 R_{*}\left(0.083 R_{*}\right)$ \\
\hline 3400 & 100 & $0.026 R_{*}\left(0.083 R_{*}\right)$ & $0.026 R_{*}\left(0.083 R_{*}\right)$ & $0.026 R_{*}\left(0.083 R_{*}\right)$ & $0.026 R_{*}\left(0.083 R_{*}\right)$ & $0.026 R_{*}\left(0.083 R_{*}\right)$ & $0.035 R_{*}\left(0.111 R_{*}\right)$ & $0.026 R_{*}\left(0.083 R_{*}\right)$ & $0.026 R_{*}\left(0.083 R_{*}\right)$ & $0.035 R_{*}\left(0.111 R_{*}\right)$ \\
\hline 3400 & 150 & $0.026 R_{*}\left(0.083 R_{*}\right)$ & $0.035 R_{*}\left(0.111 R_{*}\right)$ & $0.035 R_{*}\left(0.111 R_{*}\right)$ & $0.035 R_{*}\left(0.111 R_{*}\right)$ & $0.035 R_{*}\left(0.111 R_{*}\right)$ & $0.035 R_{*}\left(0.111 R_{*}\right)$ & $0.035 R_{*}\left(0.111 R_{*}\right)$ & $0.035 R_{*}\left(0.111 R_{*}\right)$ & $0.044 R_{*}\left(0.139 R_{*}\right)$ \\
\hline 3400 & 200 & $0.035 R_{*}\left(0.111 R_{*}\right)$ & $035 R_{*}\left(0.111 R_{*}\right)$ & $0.044 R_{*}\left(0.139 R_{*}\right)$ & $035 R_{*}\left(0.111 R_{*}\right)$ & $0.044 R_{*}\left(0.139 R_{*}\right)$ & $0.044 R_{*}\left(0.139 R_{*}\right)$ & $0.044 R_{*}\left(0.139 R_{*}\right)$ & $0.044 R_{*}\left(0.139 R_{*}\right)$ & $0.044 R_{*}\left(0.139 R_{*}\right)$ \\
\hline 3475 & 60 & $0.017 R_{*}\left(0.056 R_{*}\right)$ & $0.017 R_{*}\left(0.056 R_{*}\right)$ & $0.026 R_{*}\left(0.083 R_{*}\right)$ & $0.017 R_{*}\left(0.056 R_{*}\right)$ & $0.026 R_{*}\left(0.083 R_{*}\right)$ & $0.026 R_{*}\left(0.083 R_{*}\right)$ & $0.026 R_{*}\left(0.083 R_{*}\right)$ & $0.026 R_{*}\left(0.083 R_{*}\right)$ & $0.026 R_{*}\left(0.083 R_{*}\right)$ \\
\hline 3475 & 100 & $0.026 R_{*}\left(0.083 R_{*}\right)$ & $0.026 R_{*}\left(0.083 R_{*}\right)$ & $0.035 R_{*}\left(0.111 R_{*}\right)$ & $0.026 R_{*}\left(0.083 R_{*}\right)$ & $0.035 R_{*}\left(0.111 R_{*}\right)$ & $0.035 R_{*}\left(0.111 R_{*}\right)$ & $0.035 R_{*}\left(0.111 R_{*}\right)$ & $0.035 R_{*}\left(0.111 R_{*}\right)$ & $0.035 R_{*}\left(0.111 R_{*}\right)$ \\
\hline 3475 & 150 & $0.035 R_{*}\left(0.111 R_{*}\right)$ & $0.035 R_{*}\left(0.111 R_{*}\right)$ & $0.035 R_{*}\left(0.111 R_{*}\right)$ & $0.035 R_{*}\left(0.111 R_{*}\right)$ & $0.044 R_{*}\left(0.139 R_{*}\right)$ & $0.044 R_{*}\left(0.139 R_{*}\right)$ & $0.044 R_{*}\left(0.139 R_{*}\right)$ & $0.044 R_{*}\left(0.139 R_{*}\right)$ & $0.052 R_{*}\left(0.167 R_{*}\right)$ \\
\hline 3475 & 200 & $0.035 R_{*}\left(0.111 R_{*}\right)$ & $0.044 R_{*}\left(0.139 R_{*}\right)$ & $0.044 R_{*}\left(0.139 R_{*}\right)$ & $0.044 R_{*}\left(0.139 R_{*}\right)$ & $0.044 R_{*}\left(0.139 R_{*}\right)$ & $0.052 R_{*}\left(0.167 R_{*}\right)$ & $0.052 R_{*}\left(0.167 R_{*}\right)$ & $0.052 R_{*}\left(0.167 R_{*}\right)$ & $0.061 R_{*}\left(0.194 R_{*}\right)$ \\
\hline 3550 & 60 & $0.026 R_{*}\left(0.083 R_{*}\right)$ & $0.026 R_{*}\left(0.083 R_{*}\right)$ & $0.026 R_{*}\left(0.083 R_{*}\right)$ & $0.026 R_{*}\left(0.083 R_{*}\right)$ & $0.026 R_{*}\left(0.083 R_{*}\right)$ & $0.035 R_{*}\left(0.111 R_{*}\right)$ & $0.026 R_{*}\left(0.083 R_{*}\right)$ & $0.026 R_{*}\left(0.083 R_{*}\right)$ & $0.035 R_{*}\left(0.111 R_{*}\right)$ \\
\hline 3550 & 100 & $0.035 R_{*}\left(0.111 R_{*}\right)$ & $0.035 R_{*}\left(0.111 R_{*}\right)$ & $0.035 R_{*}\left(0.111 R_{*}\right)$ & $0.035 R_{*}\left(0.111 R_{*}\right)$ & $0.035 R_{*}\left(0.111 R_{*}\right)$ & $0.044 R_{*}\left(0.139 R_{*}\right)$ & $0.044 R_{*}\left(0.139 R_{*}\right)$ & $0.044 R_{*}\left(0.139 R_{*}\right)$ & $0.044 R_{*}\left(0.139 R_{*}\right)$ \\
\hline 3550 & 150 & $0.044 R_{*}\left(0.139 R_{*}\right)$ & $0.044 R_{*}\left(0.139 R_{*}\right)$ & $0.044 R_{*}\left(0.139 R_{*}\right)$ & $0.044 R_{*}\left(0.139 R_{*}\right)$ & $0.044 R_{*}\left(0.139 R_{*}\right)$ & $0.052 R_{*}\left(0.167 R_{*}\right)$ & $0.052 R_{*}\left(0.167 R_{*}\right)$ & $0.052 R_{*}\left(0.167 R_{*}\right)$ & $0.061 R_{*}\left(0.194 R_{*}\right)$ \\
\hline 550 & 200 & $0.044 R_{*}\left(0.139 R_{*}\right)$ & $0.052 R_{*}\left(0.167 R_{*}\right)$ & $0.052 R_{*}\left(0.167 R_{*}\right)$ & $0.052 R_{*}\left(0.167 R_{*}\right)$ & $0.052 R_{*}\left(0.167 R_{*}\right)$ & $0.061 R_{*}\left(0.194 R_{*}\right)$ & $0.061 R_{*}\left(0.194 R_{*}\right)$ & $0.070 R_{*}\left(0.222 R_{*}\right)$ & $0.079 R_{*}\left(0.250 R_{*}\right)$ \\
\hline 3625 & 60 & $0.026 R_{*}\left(0.083 R_{*}\right)$ & $0.035 R_{*}\left(0.111 R_{*}\right)$ & $0.035 R_{*}\left(0.111 R_{*}\right)$ & $0.035 R_{*}\left(0.111 R_{*}\right)$ & $0.035 R_{*}\left(0.111 R_{*}\right)$ & $0.044 R_{*}\left(0.139 R_{*}\right)$ & $0.035 R_{*}\left(0.111 R_{*}\right)$ & $0.044 R_{*}\left(0.139 R_{*}\right)$ & $0.044 R_{*}\left(0.139 R_{*}\right)$ \\
\hline 3625 & 100 & $0.044 R_{*}\left(0.139 R_{*}\right)$ & $0.044 R_{*}\left(0.139 R_{*}\right)$ & $0.052 R_{*}\left(0.167 R_{*}\right)$ & $0.052 R_{*}\left(0.167 R_{*}\right)$ & $0.052 R_{*}\left(0.167 R_{*}\right)$ & $0.061 R_{*}\left(0.194 R_{*}\right)$ & $0.061 R_{*}\left(0.194 R_{*}\right)$ & $0.070 R_{*}\left(0.222 R_{*}\right)$ & $0.079 R_{*}\left(0.250 R_{*}\right)$ \\
\hline 3625 & 150 & $0.061 R_{*}\left(0.194 R_{*}\right)$ & $0.061 R_{*}\left(0.194 R_{*}\right)$ & $0.070 R_{*}\left(0.222 R_{*}\right)$ & $0.079 R_{*}\left(0.250 R_{*}\right)$ & $0.087 R_{*}\left(0.278 R_{*}\right)$ & $0.105 R_{*}\left(0.333 R_{*}\right)$ & $\mathrm{NaN}$ & $\mathrm{NaN}$ & $\mathrm{NaN}$ \\
\hline 3625 & 200 & $0.096 R_{*}\left(0.305 R_{*}\right)$ & $0.087 R_{*}\left(0.278 R_{*}\right)$ & $0.122 R_{*}\left(0.389 R_{*}\right)$ & $\mathrm{NaN}$ & $\mathrm{NaN}$ & $\mathrm{NaN}$ & $\mathrm{NaN}$ & $\mathrm{NaN}$ & $\mathrm{NaN}$ \\
\hline
\end{tabular}

\begin{tabular}{|c|c|c|c|c|c|c|c|c|c|c|}
\hline \multicolumn{11}{|c|}{$R_{\mathrm{p}}=2.75 R_{\oplus}$} \\
\hline 3400 & 60 & $0.017 R_{*}\left(0.056 R_{*}\right)$ & $0.017 R_{*}\left(0.056 R_{*}\right)$ & $0.017 R_{*}\left(0.056 R_{*}\right)$ & $0.017 R_{*}\left(0.056 R_{*}\right)$ & $0.017 R_{*}\left(0.056 R_{*}\right)$ & $0.026 R_{*}\left(0.083 R_{*}\right)$ & $0.017 R_{*}\left(0.056 R_{*}\right)$ & $0.026 R_{*}\left(0.083 R_{*}\right)$ & $0.026 R_{*}\left(0.083 R_{*}\right)$ \\
\hline 3400 & 100 & $0.026 R_{*}\left(0.083 R_{*}\right)$ & $0.026 R_{*}\left(0.083 R_{*}\right)$ & $0.026 R_{*}\left(0.083 R_{*}\right)$ & $0.026 R_{*}\left(0.083 R_{*}\right)$ & $0.026 R_{*}\left(0.083 R_{*}\right)$ & $0.026 R_{*}\left(0.083 R_{*}\right)$ & $0.026 R_{*}\left(0.083 R_{*}\right)$ & $0.026 R_{*}\left(0.083 R_{*}\right)$ & $0.035 R_{*}\left(0.111 R_{*}\right)$ \\
\hline 3400 & 150 & $0.026 R_{*}\left(0.083 R_{*}\right)$ & $0.026 R_{*}\left(0.083 R_{*}\right)$ & $0.035 R_{*}\left(0.111 R_{*}\right)$ & $0.026 R_{*}\left(0.083 R_{*}\right)$ & $0.035 R_{*}\left(0.111 R_{*}\right)$ & $0.035 R_{*}\left(0.111 R_{*}\right)$ & $0.035 R_{*}\left(0.111 R_{*}\right)$ & $0.035 R_{*}\left(0.111 R_{*}\right)$ & $0.044 R_{*}\left(0.139 R_{*}\right)$ \\
\hline 3400 & 200 & $0.035 R_{*}\left(0.111 R_{*}\right)$ & $0.035 R_{*}\left(0.111 R_{*}\right)$ & $0.035 R_{*}\left(0.111 R_{*}\right)$ & $0.035 R_{*}\left(0.111 R_{*}\right)$ & $0.035 R_{*}\left(0.111 R_{*}\right)$ & $0.044 R_{*}\left(0.139 R_{*}\right)$ & $0.044 R_{*}\left(0.139 R_{*}\right)$ & $0.044 R_{*}\left(0.139 R_{*}\right)$ & $0.044 R_{*}\left(0.139 R_{*}\right)$ \\
\hline 3475 & 60 & $0.017 R_{*}\left(0.056 R_{*}\right)$ & $0.017 R_{*}\left(0.056 R_{*}\right)$ & $0.026 R_{*}\left(0.083 R_{*}\right)$ & $0.017 R_{*}\left(0.056 R_{*}\right)$ & $0.026 R_{*}\left(0.083 R_{*}\right)$ & $0.026 R_{*}\left(0.083 R_{*}\right)$ & $0.026 R_{*}\left(0.083 R_{*}\right)$ & $0.026 R_{*}\left(0.083 R_{*}\right)$ & $0.026 R_{*}\left(0.083 R_{*}\right)$ \\
\hline 3475 & 100 & $0.026 R_{*}\left(0.083 R_{*}\right)$ & $0.026 R_{*}\left(0.083 R_{*}\right)$ & $0.026 R_{*}\left(0.083 R_{*}\right)$ & $0.026 R_{*}\left(0.083 R_{*}\right)$ & $0.026 R_{*}\left(0.083 R_{*}\right)$ & $0.035 R_{*}\left(0.111 R_{*}\right)$ & $0.035 R_{*}\left(0.111 R_{*}\right)$ & $0.035 R_{*}\left(0.111 R_{*}\right)$ & $0.035 R_{*}\left(0.111 R_{*}\right)$ \\
\hline 3475 & 150 & $0.035 R_{*}\left(0.111 R_{*}\right)$ & $0.035 R_{*}\left(0.111 R_{*}\right)$ & $0.035 R_{*}\left(0.111 R_{*}\right)$ & $0.035 R_{*}\left(0.111 R_{*}\right)$ & $0.035 R_{*}\left(0.111 R_{*}\right)$ & $0.044 R_{*}\left(0.139 R_{*}\right)$ & $0.035 R_{*}\left(0.111 R_{*}\right)$ & $0.044 R_{*}\left(0.139 R_{*}\right)$ & $0.044 R_{*}\left(0.139 R_{*}\right)$ \\
\hline 3475 & 200 & $0.035 R_{*}\left(0.111 R_{*}\right)$ & $0.035 R_{*}\left(0.111 R_{*}\right)$ & $0.044 R_{*}\left(0.139 R_{*}\right)$ & $0.035 R_{*}\left(0.111 R_{*}\right)$ & $0.044 R_{*}\left(0.139 R_{*}\right)$ & $0.044 R_{*}\left(0.139 R_{*}\right)$ & $0.044 R_{*}\left(0.139 R_{*}\right)$ & $0.052 R_{*}\left(0.167 R_{*}\right)$ & $0.052 R_{*}\left(0.167 R_{*}\right)$ \\
\hline 3550 & 60 & $0.026 R_{*}\left(0.083 R_{*}\right)$ & $0.026 R_{*}\left(0.083 R_{*}\right)$ & $0.026 R_{*}\left(0.083 R_{*}\right)$ & $0.026 R_{*}\left(0.083 R_{*}\right)$ & $0.026 R_{*}\left(0.083 R_{*}\right)$ & $0.026 R_{*}\left(0.083 R_{*}\right)$ & $0.026 R_{*}\left(0.083 R_{*}\right)$ & $0.026 R_{*}\left(0.083 R_{*}\right)$ & $0.035 R_{*}\left(0.111 R_{*}\right)$ \\
\hline 3550 & 100 & $0.035 R_{*}\left(0.111 R_{*}\right)$ & $0.035 R_{*}\left(0.111 R_{*}\right)$ & $0.035 R_{*}\left(0.111 R_{*}\right)$ & $0.035 R_{*}\left(0.111 R_{*}\right)$ & $0.035 R_{*}\left(0.111 R_{*}\right)$ & $0.044 R_{*}\left(0.139 R_{*}\right)$ & $0.035 R_{*}\left(0.111 R_{*}\right)$ & $0.044 R_{*}\left(0.139 R_{*}\right)$ & $0.044 R_{*}\left(0.139 R_{*}\right)$ \\
\hline 3550 & 150 & $0.035 R_{*}\left(0.111 R_{*}\right)$ & $0.044 R_{*}\left(0.139 R_{*}\right)$ & $0.044 R_{*}\left(0.139 R_{*}\right)$ & $0.044 R_{*}\left(0.139 R_{*}\right)$ & $0.044 R_{*}\left(0.139 R_{*}\right)$ & $0.052 R_{*}\left(0.167 R_{*}\right)$ & $0.044 R_{*}\left(0.139 R_{*}\right)$ & $0.052 R_{*}\left(0.167 R_{*}\right)$ & $0.052 R_{*}\left(0.167 R_{*}\right)$ \\
\hline 3550 & 200 & $0.044 R_{*}\left(0.139 R_{*}\right)$ & $0.052 R_{*}\left(0.167 R_{*}\right)$ & $0.052 R_{*}\left(0.167 R_{*}\right)$ & $0.044 R_{*}\left(0.139 R_{*}\right)$ & $0.052 R_{*}\left(0.167 R_{*}\right)$ & $0.052 R_{*}\left(0.167 R_{*}\right)$ & $0.061 R_{*}\left(0.194 R_{*}\right)$ & $0.061 R_{*}\left(0.194 R_{*}\right)$ & $0.070 R_{*}\left(0.222 R_{*}\right)$ \\
\hline 3625 & 60 & $0.026 R_{*}\left(0.083 R_{*}\right)$ & $0.035 R_{*}\left(0.111 R_{*}\right)$ & $0.035 R_{*}\left(0.111 R_{*}\right)$ & $0.035 R_{*}\left(0.111 R_{*}\right)$ & $0.035 R_{*}\left(0.111 R_{*}\right)$ & $0.044 R_{*}\left(0.139 R_{*}\right)$ & $0.035 R_{*}\left(0.111 R_{*}\right)$ & $0.044 R_{*}\left(0.139 R_{*}\right)$ & $0.044 R_{*}\left(0.139 R_{*}\right)$ \\
\hline
\end{tabular}

Notes. For a direct comparison with the results from the M4V dwarf host star simulations, the values of $r_{\text {spot }}$ in brackets are the starspot size if they were on the M4V dwarf.

A114, page 18 of 23 
Table A.2. continued.

\begin{tabular}{|c|c|c|c|c|c|c|c|c|c|c|}
\hline \multirow{2}{*}{$\begin{array}{l}T_{\text {spot }} \\
(K)\end{array}$} & \multirow{2}{*}{$\begin{array}{c}\text { rms scatter } \\
\text { (ppm) }\end{array}$} & \multicolumn{3}{|c|}{$\begin{array}{c}\lambda_{\mathrm{obs}}=600 \mathrm{~nm} \\
P\end{array}$} & \multicolumn{3}{|c|}{$\begin{array}{c}\lambda_{\mathrm{obs}}=785 \mathrm{~nm} \\
P\end{array}$} & \multicolumn{3}{|c|}{$\begin{array}{c}\lambda_{\mathrm{obs}}=1000 \mathrm{~nm} \\
P\end{array}$} \\
\hline & & $2 \mathrm{~d}$ & $3 \mathrm{~d}$ & $4 \mathrm{~d}$ & $2 \mathrm{~d}$ & $3 \mathrm{~d}$ & $4 \mathrm{~d}$ & $2 \mathrm{~d}$ & $3 \mathrm{~d}$ & $4 \mathrm{~d}$ \\
\hline 3625 & 100 & $0.044 R_{*}\left(0.139 R_{*}\right)$ & $0.044 R_{*}\left(0.139 R_{*}\right)$ & $0.044 R_{*}\left(0.139 R_{*}\right)$ & $0.052 R_{*}\left(0.167 R_{*}\right)$ & $0.052 R_{*}\left(0.167 R_{*}\right)$ & $0.061 R_{*}\left(0.194 R_{*}\right)$ & $0.052 R_{*}\left(0.167 R_{*}\right)$ & $0.061 R_{*}\left(0.194 R_{*}\right)$ & $0.061 R_{*}\left(0.194 R_{*}\right)$ \\
\hline 3625 & 150 & $0.052 R_{*}\left(0.167 R_{*}\right)$ & $0.061 R_{*}\left(0.194 R_{*}\right)$ & $0.061 R_{*}\left(0.194 R_{*}\right)$ & $0.061 R_{*}\left(0.194 R_{*}\right)$ & $0.070 R_{*}\left(0.222 R_{*}\right)$ & $0.079 R_{*}\left(0.250 R_{*}\right)$ & $0.096 R_{*}\left(0.305 R_{*}\right)$ & $0.087 R_{*}\left(0.278 R_{*}\right)$ & $0.105 R_{*}\left(0.333 R_{*}\right)$ \\
\hline 3625 & 200 & $0.061 R_{*}\left(0.194 R_{*}\right)$ & $0.079 R_{*}\left(0.250 R_{*}\right)$ & $0.079 R_{*}\left(0.250 R_{*}\right)$ & $0.105 R_{*}\left(0.333 R_{*}\right)$ & $0.166 R_{*}\left(0.527 R_{*}\right)$ & $0.105 R_{*}\left(0.333 R_{*}\right)$ & $\mathrm{NaN}$ & $\mathrm{NaN}$ & $\mathrm{NaN}$ \\
\hline
\end{tabular}

$R_{\mathrm{p}}=3.00 R_{\oplus}$

\begin{tabular}{|c|c|c|c|c|c|c|c|c|c|c|}
\hline 3400 & 60 & $0.017 R_{*}\left(0.056 R_{*}\right)$ & $0.017 R_{*}\left(0.056 R_{*}\right)$ & $0.017 R_{*}\left(0.056 R_{*}\right)$ & $0.017 R_{*}\left(0.056 R_{*}\right)$ & $0.017 R_{*}\left(0.056 R_{*}\right)$ & $0.026 R_{*}\left(0.083 R_{*}\right)$ & $0.017 R_{*}\left(0.056 R_{*}\right)$ & $0.017 R_{*}\left(0.056 R_{*}\right)$ & $0.026 R_{*}\left(0.083 R_{*}\right)$ \\
\hline 3400 & 100 & $.026 R_{*}\left(0.083 R_{*}\right)$ & $0.026 R_{*}\left(0.083 R_{*}\right)$ & $0.026 R_{*}\left(0.083 R_{*}\right)$ & $0.026 R_{*}\left(0.083 R_{*}\right)$ & $0.026 R_{*}\left(0.083 R_{*}\right)$ & $0.026 R_{*}\left(0.083 R_{*}\right)$ & $0.026 R_{*}\left(0.083 R_{*}\right)$ & $0.026 R_{*}\left(0.083 R_{*}\right)$ & $0.035 R_{*}\left(0.111 R_{*}\right)$ \\
\hline 3400 & 150 & $026 R_{*}\left(0.083 R_{*}\right)$ & $0.026 R_{*}\left(0.083 R_{*}\right)$ & $0.035 R_{*}\left(0.111 R_{*}\right)$ & $026 R_{*}\left(0.083 R_{*}\right)$ & $0.035 R_{*}\left(0.111 R_{*}\right)$ & $035 R_{*}\left(0.111 R_{*}\right)$ & $.035 R_{*}\left(0.111 R_{*}\right)$ & $\left.1 R_{*}\right)$ & $\left(11 R_{*}\right)$ \\
\hline 3400 & 200 & $0.035 R_{*}\left(0.111 R_{*}\right)$ & $0.035 R_{*}\left(0.111 R_{*}\right)$ & $0.035 R_{*}\left(0.111 R_{*}\right)$ & $035 R_{*}\left(0.111 R_{*}\right)$ & $0.035 R_{*}\left(0.111 R_{*}\right)$ & $0.035 R_{*}\left(0.111 R_{*}\right)$ & $0.035 R_{*}\left(0.111 R_{*}\right)$ & $0.044 R_{*}\left(0.139 R_{*}\right)$ & $0.044 R_{*}\left(0.139 R_{*}\right)$ \\
\hline 3475 & 60 & $0.017 R_{*}\left(0.056 R_{*}\right)$ & $0.017 R_{*}\left(0.056 R_{*}\right)$ & $0.026 R_{*}\left(0.083 R_{*}\right)$ & $0.017 R_{*}\left(0.056 R_{*}\right)$ & $0.026 R_{*}\left(0.083 R_{*}\right)$ & $0.026 R_{*}\left(0.083 R_{*}\right)$ & $0.026 R_{*}\left(0.083 R_{*}\right)$ & $0.026 R_{*}\left(0.083 R_{*}\right)$ & $0.026 R_{*}\left(0.083 R_{*}\right)$ \\
\hline 3475 & 100 & $026 R_{*}\left(0.083 R_{*}\right)$ & $0.026 R_{*}\left(0.083 R_{*}\right)$ & $0.026 R_{*}\left(0.083 R_{*}\right)$ & $0.026 R_{*}\left(0.083 R_{*}\right)$ & $0.026 R_{*}\left(0.083 R_{*}\right)$ & $0.035 R_{*}\left(0.111 R_{*}\right)$ & $0.026 R_{*}\left(0.083 R_{*}\right)$ & $0.035 R_{*}\left(0.111 R_{*}\right)$ & $\left(0.111 R_{*}\right)$ \\
\hline 3475 & 150 & $0.035 R_{*}\left(0.111 R_{*}\right)$ & $0.035 R_{*}\left(0.111 R_{*}\right)$ & $0.035 R_{*}\left(0.111 R_{*}\right)$ & $0.035 R_{*}\left(0.111 R_{*}\right)$ & $0.035 R_{*}\left(0.111 R_{*}\right)$ & $0.044 R_{*}\left(0.139 R_{*}\right)$ & $0.035 R_{*}\left(0.111 R_{*}\right)$ & $0.044 R_{*}\left(0.139 R_{*}\right)$ & $0.044 R_{*}\left(0.139 R_{*}\right)$ \\
\hline 3475 & 200 & $0.035 R_{*}\left(0.111 R_{*}\right)$ & $0.035 R_{*}\left(0.111 R_{*}\right)$ & $0.044 R_{*}\left(0.139 R_{*}\right)$ & $0.044 R_{*}\left(0.139 R_{*}\right)$ & $0.044 R_{*}\left(0.139 R_{*}\right)$ & $0.044 R_{*}\left(0.139 R_{*}\right)$ & $0.044 R_{*}\left(0.139 R_{*}\right)$ & $0.044 R_{*}\left(0.139 R_{*}\right)$ & $\left.0.167 R_{*}\right)$ \\
\hline 3550 & 60 & $026 R_{*}\left(0.083 R_{*}\right)$ & $0.026 R_{*}\left(0.083 R_{*}\right)$ & $0.026 R_{*}\left(0.083 R_{*}\right)$ & $0.026 R_{*}\left(0.083 R_{*}\right)$ & $0.026 R_{*}\left(0.083 R_{*}\right)$ & $0.026 R_{*}\left(0.083 R_{*}\right)$ & $0.026 R_{*}\left(0.083 R_{*}\right)$ & $0.026 R_{*}\left(0.083 R_{*}\right)$ & $0.026 R_{*}\left(0.083 R_{*}\right)$ \\
\hline 3550 & 100 & $0.026 R_{*}\left(0.083 R_{*}\right)$ & $0.035 R_{*}\left(0.111 R_{*}\right)$ & $0.035 R_{*}\left(0.111 R_{*}\right)$ & $0.035 R_{*}\left(0.111 R_{*}\right)$ & $0.035 R_{*}\left(0.111 R_{*}\right)$ & $0.035 R_{*}\left(0.111 R_{*}\right)$ & $0.035 R_{*}\left(0.111 R_{*}\right)$ & $0.044 R_{*}\left(0.139 R_{*}\right)$ & $0.044 R_{*}\left(0.139 R_{*}\right)$ \\
\hline 3550 & 150 & $0.035 R_{*}\left(0.111 R_{*}\right)$ & $0.044 R_{*}\left(0.139 R_{*}\right)$ & $0.044 R_{*}\left(0.139 R_{*}\right)$ & $0.044 R_{*}\left(0.139 R_{*}\right)$ & $0.044 R_{*}\left(0.139 R_{*}\right)$ & $0.044 R_{*}\left(0.139 R_{*}\right)$ & $0.044 R_{*}\left(0.139 R_{*}\right)$ & $0.044 R_{*}\left(0.139 R_{*}\right)$ & $0.052 R_{*}\left(0.167 R_{*}\right)$ \\
\hline 3550 & 200 & $0.044 R_{*}\left(0.139 R_{*}\right)$ & $0.044 R_{*}\left(0.139 R_{*}\right)$ & $0.044 R_{*}\left(0.139 R_{*}\right)$ & $0.052 R_{*}\left(0.167 R_{*}\right)$ & $0.052 R_{*}\left(0.167 R_{*}\right)$ & $0.052 R_{*}\left(0.167 R_{*}\right)$ & $0.052 R_{*}\left(0.167 R_{*}\right)$ & $0.052 R_{*}\left(0.167 R_{*}\right)$ & $0.061 R_{*}\left(0.194 R_{*}\right)$ \\
\hline 3625 & 60 & $0.026 R_{*}\left(0.083 R_{*}\right)$ & $0.035 R_{*}\left(0.111 R_{*}\right)$ & $0.035 R_{*}\left(0.111 R_{*}\right)$ & $0.035 R_{*}\left(0.111 R_{*}\right)$ & $0.035 R_{*}\left(0.111 R_{*}\right)$ & $0.035 R_{*}\left(0.111 R_{*}\right)$ & $0.035 R_{*}\left(0.111 R_{*}\right)$ & $0.044 R_{*}\left(0.139 R_{*}\right)$ & $0.044 R_{*}\left(0.139 R_{*}\right)$ \\
\hline 3625 & 100 & $0.044 R_{*}\left(0.139 R_{*}\right)$ & $0.044 R_{*}\left(0.139 R_{*}\right)$ & $0.052 R_{*}\left(0.167 R_{*}\right)$ & $0.052 R_{*}\left(0.167 R_{*}\right)$ & $0.052 R_{*}\left(0.167 R_{*}\right)$ & $0.052 R_{*}\left(0.167 R_{*}\right)$ & $0.052 R_{*}\left(0.167 R_{*}\right)$ & $0.061 R_{*}\left(0.194 R_{*}\right)$ & $0.061 R_{*}\left(0.194 R_{*}\right)$ \\
\hline 3625 & 150 & $0.052 R_{*}\left(0.167 R_{*}\right)$ & $0.052 R_{*}\left(0.167 R_{*}\right)$ & $0.061 R_{*}\left(0.194 R_{*}\right)$ & $0.061 R_{*}\left(0.194 R_{*}\right)$ & $0.061 R_{*}\left(0.194 R_{*}\right)$ & $0.061 R_{*}\left(0.194 R_{*}\right)$ & $0.070 R_{*}\left(0.222 R_{*}\right)$ & $0.079 R_{*}\left(0.250 R_{*}\right)$ & $0.087 R_{*}\left(0.278 R_{*}\right)$ \\
\hline 3625 & 200 & $0.061 R_{*}\left(0.194 R_{*}\right)$ & $0.061 R_{*}\left(0.194 R_{*}\right)$ & $0.070 R_{*}\left(0.222 R_{*}\right)$ & $0.079 R_{*}\left(0.250 R_{*}\right)$ & $0.079 R_{*}\left(0.250 R_{*}\right)$ & $0.087 R_{*}\left(0.278 R_{*}\right)$ & $0.105 R_{*}\left(0.333 R_{*}\right)$ & $\mathrm{NaN}$ & $\mathrm{NaN}$ \\
\hline
\end{tabular}

\begin{tabular}{rrrrrrr}
3625 & 200 & $0.061 R_{*}\left(0.194 R_{*}\right)$ & $0.061 R_{*}\left(0.194 R_{*}\right)$ & $0.070 R_{*}\left(0.222 R_{*}\right)$ & $0.079 R_{*}\left(0.250 R_{*}\right)$ & $0.079 R_{*}$ \\
\hline & & $R_{\mathrm{p}}=3.25 R_{\oplus}$
\end{tabular}

\begin{tabular}{|c|c|c|c|c|c|c|c|c|c|c|}
\hline 3400 & 60 & $0.017 R_{*}\left(0.056 R_{*}\right)$ & $0.017 R_{*}\left(0.056 R_{*}\right)$ & $0.017 R_{*}\left(0.056 R_{*}\right)$ & $0.017 R_{*}\left(0.056 R_{*}\right)$ & $0.017 R_{*}\left(0.056 R_{*}\right)$ & $0.017 R_{*}\left(0.056 R_{*}\right)$ & $0.017 R_{*}\left(0.056 R_{*}\right)$ & $0.017 R_{*}\left(0.056 R_{*}\right)$ & $0.026 R_{*}\left(0.083 R_{*}\right)$ \\
\hline 3400 & 100 & $.026 R_{*}\left(0.083 R_{*}\right)$ & $0.026 R_{*}\left(0.083 R_{*}\right)$ & $0.026 R_{*}\left(0.083 R_{*}\right)$ & $0.026 R_{*}\left(0.083 R_{*}\right)$ & $0.026 R_{*}\left(0.083 R_{*}\right)$ & $0.026 R_{*}\left(0.083 R_{*}\right)$ & $0.026 R_{*}\left(0.083 R_{*}\right)$ & $0.026 R_{*}\left(0.083 R_{*}\right)$ & $0.026 R_{*}\left(0.083 R_{*}\right)$ \\
\hline 3400 & 150 & $026 R_{*}\left(0.083 R_{*}\right)$ & $0.026 R_{*}\left(0.083 R_{*}\right)$ & $0.026 R_{*}\left(0.083 R_{*}\right)$ & $.026 R_{*}\left(0.083 R_{*}\right)$ & $0.035 R_{*}\left(0.111 R_{*}\right)$ & $035 R_{*}\left(0.111 R_{*}\right)$ & $.035 R_{*}\left(0.111 R_{*}\right)$ & $R_{*}\left(0.111 R_{*}\right)$ & $\left.0.111 R_{*}\right)$ \\
\hline 3400 & 200 & $026 R_{*}\left(0.083 R_{*}\right)$ & $0.035 R_{*}\left(0.111 R_{*}\right)$ & $0.035 R_{*}\left(0.111 R_{*}\right)$ & $0.035 R_{*}\left(0.111 R_{*}\right)$ & $0.035 R_{*}\left(0.111 R_{*}\right)$ & $0.035 R_{*}\left(0.111 R_{*}\right)$ & $0.035 R_{*}\left(0.111 R_{*}\right)$ & $0.044 R_{*}\left(0.139 R_{*}\right)$ & $0.044 R_{*}\left(0.139 R_{*}\right)$ \\
\hline 3475 & 60 & $017 R_{*}\left(0.056 R_{*}\right)$ & $\left(0.056 R_{*}\right)$ & $0.017 R_{*}\left(0.056 R_{*}\right)$ & $017 R_{*}\left(0.056 R_{*}\right)$ & $0.017 R_{*}\left(0.056 R_{*}\right)$ & $0.026 R_{*}\left(0.083 R_{*}\right)$ & $0.026 R_{*}\left(0.083 R_{*}\right)$ & $0.026 R_{*}\left(0.083 R_{*}\right)$ & $0.026 R_{*}\left(0.083 R_{*}\right)$ \\
\hline 3475 & 100 & $26 R_{*}\left(0.083 R_{*}\right)$ & $0.026 R_{*}\left(0.083 R_{*}\right)$ & $0.026 R_{*}\left(0.083 R_{*}\right)$ & $\left..083 R_{*}\right)$ & $0.026 R_{*}\left(0.083 R_{*}\right)$ & $0.035 R_{*}\left(0.111 R_{*}\right)$ & $0.035 R_{*}\left(0.111 R_{*}\right)$ & $0.035 R_{*}\left(0.111 R_{*}\right)$ & $\left.0.111 R_{*}\right)$ \\
\hline 3475 & 150 & $0.035 R_{*}\left(0.111 R_{*}\right)$ & $0.035 R_{*}\left(0.111 R_{*}\right)$ & $0.035 R_{*}\left(0.111 R_{*}\right)$ & $0.035 R_{*}\left(0.111 R_{*}\right)$ & $0.035 R_{*}\left(0.111 R_{*}\right)$ & $0.035 R_{*}\left(0.111 R_{*}\right)$ & $0.035 R_{*}\left(0.111 R_{*}\right)$ & $0.035 R_{*}\left(0.111 R_{*}\right)$ & $\left.139 R_{*}\right)$ \\
\hline 3475 & 200 & $0.035 R_{*}\left(0.111 R_{*}\right)$ & $0.035 R_{*}\left(0.111 R_{*}\right)$ & $0.035 R_{*}\left(0.111 R_{*}\right)$ & $0.035 R_{*}\left(0.111 R_{*}\right)$ & $0.044 R_{*}\left(0.139 R_{*}\right)$ & $0.044 R_{*}\left(0.139 R_{*}\right)$ & $0.044 R_{*}\left(0.139 R_{*}\right)$ & $*\left(0.139 R_{*}\right)$ & $\left.67 R_{*}\right)$ \\
\hline 3550 & 60 & $026 R_{*}\left(0.083 R_{*}\right)$ & $0.026 R_{*}\left(0.083 R_{*}\right)$ & $0.026 R_{*}\left(0.083 R_{*}\right)$ & $0.026 R_{*}\left(0.083 R_{*}\right)$ & $0.026 R_{*}\left(0.083 R_{*}\right)$ & $0.026 R_{*}\left(0.083 R_{*}\right)$ & $0.026 R_{*}\left(0.083 R_{*}\right)$ & $0.026 R_{*}\left(0.083 R_{*}\right)$ & $0.026 R_{*}\left(0.083 R_{*}\right)$ \\
\hline 3550 & 100 & $0.026 R_{*}\left(0.083 R_{*}\right)$ & $0.026 R_{*}\left(0.083 R_{*}\right)$ & $0.035 R_{*}\left(0.111 R_{*}\right)$ & $0.035 R_{*}\left(0.111 R_{*}\right)$ & $0.035 R_{*}\left(0.111 R_{*}\right)$ & $0.035 R_{*}\left(0.111 R_{*}\right)$ & $0.035 R_{*}\left(0.111 R_{*}\right)$ & $0.035 R_{*}\left(0.111 R_{*}\right)$ & $0.044 R_{*}\left(0.139 R_{*}\right)$ \\
\hline 3550 & 150 & $0.035 R_{*}\left(0.111 R_{*}\right)$ & $0.035 R_{*}\left(0.111 R_{*}\right)$ & $0.044 R_{*}\left(0.139 R_{*}\right)$ & $0.035 R_{*}\left(0.111 R_{*}\right)$ & $0.044 R_{*}\left(0.139 R_{*}\right)$ & $0.044 R_{*}\left(0.139 R_{*}\right)$ & $0.044 R_{*}\left(0.139 R_{*}\right)$ & $0.044 R_{*}\left(0.139 R_{*}\right)$ & $0.052 R_{*}\left(0.167 R_{*}\right)$ \\
\hline 3550 & 200 & $0.044 R_{*}\left(0.139 R_{*}\right)$ & $0.044 R_{*}\left(0.139 R_{*}\right)$ & $0.044 R_{*}\left(0.139 R_{*}\right)$ & $0.052 R_{*}\left(0.167 R_{*}\right)$ & $0.044 R_{*}\left(0.139 R_{*}\right)$ & $0.052 R_{*}\left(0.167 R_{*}\right)$ & $0.052 R_{*}\left(0.167 R_{*}\right)$ & $0.052 R_{*}\left(0.167 R_{*}\right)$ & $0.061 R_{*}\left(0.194 R_{*}\right)$ \\
\hline 3625 & 60 & $0.026 R_{*}\left(0.083 R_{*}\right)$ & $0.035 R_{*}\left(0.111 R_{*}\right)$ & $0.035 R_{*}\left(0.111 R_{*}\right)$ & $0.035 R_{*}\left(0.111 R_{*}\right)$ & $0.035 R_{*}\left(0.111 R_{*}\right)$ & $0.035 R_{*}\left(0.111 R_{*}\right)$ & $0.035 R_{*}\left(0.111 R_{*}\right)$ & $0.035 R_{*}\left(0.111 R_{*}\right)$ & $0.044 R_{*}\left(0.139 R_{*}\right)$ \\
\hline 3625 & 100 & $0.044 R_{*}\left(0.139 R_{*}\right)$ & $0.044 R_{*}\left(0.139 R_{*}\right)$ & $0.044 R_{*}\left(0.139 R_{*}\right)$ & $0.044 R_{*}\left(0.139 R_{*}\right)$ & $0.052 R_{*}\left(0.167 R_{*}\right)$ & $0.052 R_{*}\left(0.167 R_{*}\right)$ & $0.052 R_{*}\left(0.167 R_{*}\right)$ & $0.052 R_{*}\left(0.167 R_{*}\right)$ & $0.061 R_{*}\left(0.194 R_{*}\right)$ \\
\hline 3625 & 150 & $0.052 R_{*}\left(0.167 R_{*}\right)$ & $0.052 R_{*}\left(0.167 R_{*}\right)$ & $0.061 R_{*}\left(0.194 R_{*}\right)$ & $0.052 R_{*}\left(0.167 R_{*}\right)$ & $0.061 R_{*}\left(0.194 R_{*}\right)$ & $0.061 R_{*}\left(0.194 R_{*}\right)$ & $0.061 R_{*}\left(0.194 R_{*}\right)$ & $0.070 R_{*}\left(0.222 R_{*}\right)$ & $0.070 R_{*}\left(0.222 R_{*}\right)$ \\
\hline 3625 & 200 & $0.061 R_{*}\left(0.194 R_{*}\right)$ & $0.061 R_{*}\left(0.194 R_{*}\right)$ & $0.061 R_{*}\left(0.194 R_{*}\right)$ & $0.070 R_{*}\left(0.222 R_{*}\right)$ & $0.070 R_{*}\left(0.222 R_{*}\right)$ & $0.079 R_{*}\left(0.250 R_{*}\right)$ & $0.087 R_{*}\left(0.278 R_{*}\right)$ & $0.096 R_{*}\left(0.305 R_{*}\right)$ & $0.113 R_{*}\left(0.361 R_{*}\right)$ \\
\hline
\end{tabular}

$R_{\mathrm{p}}=3.50 R_{\oplus}$

\begin{tabular}{|c|c|c|c|c|c|c|c|c|c|c|}
\hline 3400 & 60 & $0.017 R_{*}\left(0.056 R_{*}\right)$ & $0.017 R_{*}\left(0.056 R_{*}\right)$ & $0.017 R_{*}\left(0.056 R_{*}\right)$ & $0.017 R_{*}\left(0.056 R_{*}\right)$ & $0.017 R_{*}\left(0.056 R_{*}\right)$ & $0.017 R_{*}\left(0.056 R_{*}\right)$ & $0.017 R_{*}\left(0.056 R_{*}\right)$ & $0.017 R_{*}\left(0.056 R_{*}\right)$ & $0.017 R_{*}\left(0.056 R_{*}\right)$ \\
\hline 3400 & 100 & $026 R_{*}\left(0.083 R_{*}\right)$ & $0.026 R_{*}\left(0.083 R_{*}\right)$ & $0.026 R_{*}\left(0.083 R_{*}\right)$ & $0.026 R_{*}\left(0.083 R_{*}\right)$ & $026 R_{*}\left(0.083 R_{*}\right)$ & $0.026 R_{*}\left(0.083 R_{*}\right)$ & $0.026 R_{*}\left(0.083 R_{*}\right)$ & $0.026 R_{*}\left(0.083 R_{*}\right)$ & $\left..083 R_{*}\right)$ \\
\hline 3400 & 150 & $026 R_{*}\left(0.083 R_{*}\right)$ & $026 R_{*}\left(0.083 R_{*}\right)$ & $0.026 R_{*}\left(0.083 R_{*}\right)$ & $026 R_{*}\left(0.083 R_{*}\right)$ & $026 R_{*}\left(0.083 R_{*}\right)$ & $035 R_{*}\left(0.111 R_{*}\right)$ & $035 R_{*}\left(0.111 R_{*}\right)$ & $0.035 R_{*}\left(0.111 R_{*}\right)$ & $\left.111 R_{*}\right)$ \\
\hline 3400 & 200 & $0.035 R_{*}\left(0.111 R_{*}\right)$ & $0.035 R_{*}\left(0.111 R_{*}\right)$ & $0.035 R_{*}\left(0.111 R_{*}\right)$ & $.035 R_{*}\left(0.111 R_{*}\right)$ & $0.035 R_{*}\left(0.111 R_{*}\right)$ & $0.035 R_{*}\left(0.111 R_{*}\right)$ & $0.035 R_{*}\left(0.111 R_{*}\right)$ & $0.035 R_{*}\left(0.111 R_{*}\right)$ & $0.035 R_{*}\left(0.111 R_{*}\right)$ \\
\hline 3475 & 60 & $0.017 R_{*}\left(0.056 R_{*}\right)$ & $0.017 R_{*}\left(0.056 R_{*}\right)$ & $0.017 R_{*}\left(0.056 R_{*}\right)$ & $0.017 R_{*}\left(0.056 R_{*}\right)$ & $0.017 R_{*}\left(0.056 R_{*}\right)$ & $0.017 R_{*}\left(0.056 R_{*}\right)$ & $0.026 R_{*}\left(0.083 R_{*}\right)$ & $0.026 R_{*}\left(0.083 R_{*}\right)$ & $0.026 R_{*}\left(0.083 R_{*}\right)$ \\
\hline 3475 & 100 & $0.026 R_{*}\left(0.083 R_{*}\right)$ & $0.026 R_{*}\left(0.083 R_{*}\right)$ & $0.026 R_{*}\left(0.083 R_{*}\right)$ & $0.026 R_{*}\left(0.083 R_{*}\right)$ & $0.026 R_{*}\left(0.083 R_{*}\right)$ & $0.035 R_{*}\left(0.111 R_{*}\right)$ & $0.035 R_{*}\left(0.111 R_{*}\right)$ & $0.035 R_{*}\left(0.111 R_{*}\right)$ & $0.035 R_{*}\left(0.111 R_{*}\right)$ \\
\hline 3475 & 150 & $0.026 R_{*}\left(0.083 R_{*}\right)$ & $0.026 R_{*}\left(0.083 R_{*}\right)$ & $0.035 R_{*}\left(0.111 R_{*}\right)$ & $0.035 R_{*}\left(0.111 R_{*}\right)$ & $0.035 R_{*}\left(0.111 R_{*}\right)$ & $0.035 R_{*}\left(0.111 R_{*}\right)$ & $0.035 R_{*}\left(0.111 R_{*}\right)$ & $0.035 R_{*}\left(0.111 R_{*}\right)$ & $0.035 R_{*}\left(0.111 R_{*}\right)$ \\
\hline 3475 & 200 & $0.035 R_{*}\left(0.111 R_{*}\right)$ & $0.035 R_{*}\left(0.111 R_{*}\right)$ & $0.035 R_{*}\left(0.111 R_{*}\right)$ & $0.035 R_{*}\left(0.111 R_{*}\right)$ & $0.044 R_{*}\left(0.139 R_{*}\right)$ & $0.044 R_{*}\left(0.139 R_{*}\right)$ & $0.044 R_{*}\left(0.139 R_{*}\right)$ & $0.044 R_{*}\left(0.139 R_{*}\right)$ & $0.044 R_{*}\left(0.139 R_{*}\right)$ \\
\hline 3550 & 60 & $017 R_{*}\left(0.056 R_{*}\right)$ & $0.017 R_{*}\left(0.056 R_{*}\right)$ & $0.026 R_{*}\left(0.083 R_{*}\right)$ & $0.026 R_{*}\left(0.083 R_{*}\right)$ & $0.026 R_{*}\left(0.083 R_{*}\right)$ & $0.026 R_{*}\left(0.083 R_{*}\right)$ & $0.026 R_{*}\left(0.083 R_{*}\right)$ & $0.026 R_{*}\left(0.083 R_{*}\right)$ & $0.026 R_{*}\left(0.083 R_{*}\right)$ \\
\hline 3550 & 100 & $0.026 R_{*}\left(0.083 R_{*}\right)$ & $0.035 R_{*}\left(0.111 R_{*}\right)$ & $0.035 R_{*}\left(0.111 R_{*}\right)$ & $0.035 R_{*}\left(0.111 R_{*}\right)$ & $0.035 R_{*}\left(0.111 R_{*}\right)$ & $0.035 R_{*}\left(0.111 R_{*}\right)$ & $0.035 R_{*}\left(0.111 R_{*}\right)$ & $0.035 R_{*}\left(0.111 R_{*}\right)$ & $0.044 R_{*}\left(0.139 R_{*}\right)$ \\
\hline 3550 & 150 & $0.035 R_{*}\left(0.111 R_{*}\right)$ & $0.035 R_{*}\left(0.111 R_{*}\right)$ & $0.035 R_{*}\left(0.111 R_{*}\right)$ & $0.044 R_{*}\left(0.139 R_{*}\right)$ & $0.044 R_{*}\left(0.139 R_{*}\right)$ & $0.044 R_{*}\left(0.139 R_{*}\right)$ & $0.044 R_{*}\left(0.139 R_{*}\right)$ & $0.052 R_{*}\left(0.167 R_{*}\right)$ & $0.044 R_{*}\left(0.139 R_{*}\right)$ \\
\hline 3550 & 200 & $0.044 R_{*}\left(0.139 R_{*}\right)$ & $0.044 R_{*}\left(0.139 R_{*}\right)$ & $0.044 R_{*}\left(0.139 R_{*}\right)$ & $0.044 R_{*}\left(0.139 R_{*}\right)$ & $0.044 R_{*}\left(0.139 R_{*}\right)$ & $0.052 R_{*}\left(0.167 R_{*}\right)$ & $0.052 R_{*}\left(0.167 R_{*}\right)$ & $0.052 R_{*}\left(0.167 R_{*}\right)$ & $0.052 R_{*}\left(0.167 R_{*}\right)$ \\
\hline 3625 & 60 & $0.026 R_{*}\left(0.083 R_{*}\right)$ & $0.026 R_{*}\left(0.083 R_{*}\right)$ & $0.035 R_{*}\left(0.111 R_{*}\right)$ & $0.035 R_{*}\left(0.111 R_{*}\right)$ & $0.035 R_{*}\left(0.111 R_{*}\right)$ & $0.035 R_{*}\left(0.111 R_{*}\right)$ & $0.035 R_{*}\left(0.111 R_{*}\right)$ & $0.035 R_{*}\left(0.111 R_{*}\right)$ & $0.035 R_{*}\left(0.111 R_{*}\right)$ \\
\hline 3625 & 100 & $0.035 R_{*}\left(0.111 R_{*}\right)$ & $0.044 R_{*}\left(0.139 R_{*}\right)$ & $0.044 R_{*}\left(0.139 R_{*}\right)$ & $0.044 R_{*}\left(0.139 R_{*}\right)$ & $0.044 R_{*}\left(0.139 R_{*}\right)$ & $0.052 R_{*}\left(0.167 R_{*}\right)$ & $0.052 R_{*}\left(0.167 R_{*}\right)$ & $0.052 R_{*}\left(0.167 R_{*}\right)$ & $0.052 R_{*}\left(0.167 R_{*}\right)$ \\
\hline 3625 & 150 & $0.052 R_{*}\left(0.167 R_{*}\right)$ & $0.052 R_{*}\left(0.167 R_{*}\right)$ & $0.052 R_{*}\left(0.167 R_{*}\right)$ & $0.061 R_{*}\left(0.194 R_{*}\right)$ & $0.052 R_{*}\left(0.167 R_{*}\right)$ & $0.061 R_{*}\left(0.194 R_{*}\right)$ & $0.061 R_{*}\left(0.194 R_{*}\right)$ & $0.070 R_{*}\left(0.222 R_{*}\right)$ & $0.070 R_{*}\left(0.222 R_{*}\right)$ \\
\hline 3625 & 200 & $0.052 R_{*}\left(0.167 R_{*}\right)$ & $0.061 R_{*}\left(0.194 R_{*}\right)$ & $0.070 R_{*}\left(0.222 R_{*}\right)$ & $0.061 R_{*}\left(0.194 R_{*}\right)$ & $0.070 R_{*}\left(0.222 R_{*}\right)$ & $0.070 R_{*}\left(0.222 R_{*}\right)$ & $0.079 R_{*}\left(0.250 R_{*}\right)$ & $0.079 R_{*}\left(0.250 R_{*}\right)$ & $0.096 R_{*}\left(0.305 R_{*}\right)$ \\
\hline
\end{tabular}

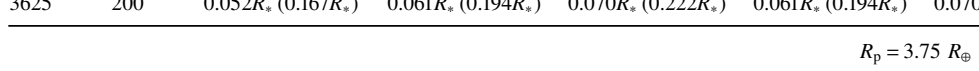

\begin{tabular}{|c|c|c|c|c|c|c|c|c|c|c|}
\hline 3400 & 60 & $0.017 R_{*}\left(0.056 R_{*}\right)$ & $0.017 R_{*}\left(0.056 R_{*}\right)$ & $0.017 R_{*}\left(0.056 R_{*}\right)$ & $0.017 R_{*}\left(0.056 R_{*}\right)$ & $0.017 R_{*}\left(0.056 R_{*}\right)$ & $0.017 R_{*}\left(0.056 R_{*}\right)$ & $0.017 R_{*}\left(0.056 R_{*}\right)$ & $0.017 R_{*}\left(0.056 R_{*}\right)$ & $0.026 R_{*}\left(0.083 R_{*}\right)$ \\
\hline 3400 & 100 & $0.026 R_{*}\left(0.083 R_{*}\right)$ & $0.026 R_{*}\left(0.083 R_{*}\right)$ & $0.026 R_{*}\left(0.083 R_{*}\right)$ & $0.026 R_{*}\left(0.083 R_{*}\right)$ & $0.026 R_{*}\left(0.083 R_{*}\right)$ & $0.026 R_{*}\left(0.083 R_{*}\right)$ & $0.026 R_{*}\left(0.083 R_{*}\right)$ & $0.026 R_{*}\left(0.083 R_{*}\right)$ & $0.026 R_{*}\left(0.083 R_{*}\right)$ \\
\hline 3400 & 150 & $.026 R_{*}\left(0.083 R_{*}\right)$ & $0.026 R_{*}\left(0.083 R_{*}\right)$ & $0.026 R_{*}\left(0.083 R_{*}\right)$ & $0.035 R_{*}\left(0.111 R_{*}\right)$ & $0.026 R_{*}\left(0.083 R_{*}\right)$ & $0.035 R_{*}\left(0.111 R_{*}\right)$ & $0.035 R_{*}\left(0.111 R_{*}\right)$ & $0.035 R_{*}\left(0.111 R_{*}\right)$ & $\left.0.111 R_{*}\right)$ \\
\hline 3400 & 200 & $035 R_{*}\left(0.111 R_{*}\right)$ & $.035 R_{*}\left(0.111 R_{*}\right)$ & $0.035 R_{*}($ & $\left..111 R_{*}\right)$ & $0.035 R_{*}\left(0.111 R_{*}\right)$ & $\left(0.111 R_{*}\right)$ & $0.035 R_{*}\left(0.111 R_{*}\right)$ & $0.035 R_{*}\left(0.111 R_{*}\right)$ & $0.035 R_{*}\left(0.111 R_{*}\right)$ \\
\hline 3475 & 60 & $\left.56 R_{*}\right)$ & $0.017 R_{*}\left(0.056 R_{*}\right)$ & $0.017 R_{*}\left(0.056 R_{*}\right)$ & $0.026 R_{*}\left(0.083 R_{*}\right)$ & $0.026 R_{*}\left(0.083 R_{*}\right)$ & $0.026 R_{*}\left(0.083 R_{*}\right)$ & $0.026 R_{*}\left(0.083 R_{*}\right)$ & $0.026 R_{*}\left(0.083 R_{*}\right)$ & $0.026 R_{*}\left(0.083 R_{*}\right)$ \\
\hline 3475 & 100 & $0.026 R_{*}\left(0.083 R_{*}\right)$ & $0.026 R_{*}\left(0.083 R_{*}\right)$ & $0.026 r$ & $0.026 R_{*}($ & $0.026 R_{*}\left(0.083 R_{*}\right)$ & $0.026 R_{*}$ & $0.035 R_{*}$ & 0.026 & $\left.11 R_{*}\right)$ \\
\hline 3475 & 150 & $0.026 R_{*}\left(0.083 R_{*}\right)$ & $0.035 R_{*}\left(0.111 R_{*}\right)$ & $0.035 R_{*}\left(0.111 R_{*}\right)$ & $0.035 R_{*}\left(0.111 R_{*}\right)$ & $0.035 R_{*}\left(0.111 R_{*}\right)$ & $0.035 R_{*}\left(0.111 R_{*}\right)$ & $0.035 R_{*}\left(0.111 R_{*}\right)$ & $0.035 R_{*}\left(0.111 R_{*}\right)$ & $0.044 R_{*}\left(0.139 R_{*}\right)$ \\
\hline 3475 & 200 & $035 R_{*}\left(0.111 R_{*}\right)$ & $0.035 R_{*}\left(0.111 R_{*}\right)$ & $0.035 R_{*}\left(0.111 R_{*}\right)$ & $0.035 R_{*}\left(0.111 R_{*}\right)$ & $0.035 R_{*}\left(0.111 R_{*}\right)$ & $0.035 R_{*}\left(0.111 R_{*}\right)$ & $0.044 R_{*}\left(0.139 R_{*}\right)$ & $0.044 R_{*}\left(0.139 R_{*}\right)$ & $0.044 R_{*}\left(0.139 R_{*}\right)$ \\
\hline 3550 & 60 & $0.026 R_{*}\left(0.083 R_{*}\right)$ & $0.026 R_{*}\left(0.083 R_{*}\right)$ & $0.026 R_{*}\left(0.083 R_{*}\right)$ & $0.026 R_{*}\left(0.083 R_{*}\right)$ & $0.026 R_{*}\left(0.083 R_{*}\right)$ & $0.026 R_{*}\left(0.083 R_{*}\right)$ & $0.026 R_{*}\left(0.083 R_{*}\right)$ & $0.026 R_{*}\left(0.083 R_{*}\right)$ & $0.026 R_{*}\left(0.083 R_{*}\right)$ \\
\hline 3550 & 100 & $0.026 R_{*}\left(0.083 R_{*}\right)$ & $0.026 R_{*}\left(0.083 R_{*}\right)$ & $0.035 R_{*}\left(0.111 R_{*}\right)$ & $0.035 R_{*}\left(0.111 R_{*}\right)$ & $0.035 R_{*}\left(0.111 R_{*}\right)$ & $0.035 R_{*}\left(0.111 R_{*}\right)$ & $0.035 R_{*}\left(0.111 R_{*}\right)$ & $0.035 R_{*}\left(0.111 R_{*}\right)$ & $0.035 R_{*}\left(0.111 R_{*}\right)$ \\
\hline 3550 & 150 & $0.035 R_{*}\left(0.111 R_{*}\right)$ & $0.035 R_{*}\left(0.111 R_{*}\right)$ & $0.035 R_{*}\left(0.111 R_{*}\right)$ & $0.044 R_{*}\left(0.139 R_{*}\right)$ & $0.044 R_{*}\left(0.139 R_{*}\right)$ & $0.044 R_{*}\left(0.139 R_{*}\right)$ & $0.044 R_{*}\left(0.139 R_{*}\right)$ & $0.044 R_{*}\left(0.139 R_{*}\right)$ & $0.052 R_{*}\left(0.167 R_{*}\right)$ \\
\hline
\end{tabular}


A\&A 630, A114 (2019)

Table A.2. continued.

\begin{tabular}{|c|c|c|c|c|c|c|c|c|c|c|}
\hline \multirow{2}{*}{$\begin{array}{l}T_{\text {spot }} \\
(K)\end{array}$} & \multirow{2}{*}{$\begin{array}{l}\text { rms scatter } \\
\text { (ppm) }\end{array}$} & \multicolumn{3}{|c|}{$\begin{array}{c}\lambda_{\mathrm{obs}}=600 \mathrm{~nm} \\
P\end{array}$} & \multicolumn{3}{|c|}{$\begin{array}{c}\lambda_{\mathrm{obs}}=785 \mathrm{~nm} \\
P\end{array}$} & \multicolumn{3}{|c|}{$\begin{array}{c}\lambda_{\mathrm{obs}}=1000 \mathrm{~nm} \\
P\end{array}$} \\
\hline & & $2 \mathrm{~d}$ & $3 \mathrm{~d}$ & $4 \mathrm{~d}$ & $2 \mathrm{~d}$ & $3 \mathrm{~d}$ & $4 \mathrm{~d}$ & $2 \mathrm{~d}$ & $3 \mathrm{~d}$ & $4 \mathrm{~d}$ \\
\hline 3550 & 200 & $0.044 R_{*}\left(0.139 R_{*}\right)$ & $0.035 R_{*}\left(0.111 R_{*}\right)$ & $0.044 R_{*}\left(0.139 R_{*}\right)$ & $0.044 R_{*}\left(0.139 R_{*}\right)$ & $0.044 R_{*}\left(0.139 R_{*}\right)$ & $0.044 R_{*}\left(0.139 R_{*}\right)$ & $0.052 R_{*}\left(0.167 R_{*}\right)$ & $0.052 R_{*}\left(0.167 R_{*}\right)$ & $0.052 R_{*}\left(0.167 R_{*}\right)$ \\
\hline 3625 & 60 & $0.026 R_{*}\left(0.083 R_{*}\right)$ & $0.026 R_{*}\left(0.083 R_{*}\right)$ & $0.035 R_{*}\left(0.111 R_{*}\right)$ & $0.035 R_{*}\left(0.111 R_{*}\right)$ & $0.035 R_{*}\left(0.111 R_{*}\right)$ & $0.035 R_{*}\left(0.111 R_{*}\right)$ & $0.035 R_{*}\left(0.111 R_{*}\right)$ & $0.035 R_{*}\left(0.111 R_{*}\right)$ & $0.044 R_{*}\left(0.139 R_{*}\right)$ \\
\hline 3625 & 100 & $0.044 R_{*}\left(0.139 R_{*}\right)$ & $0.044 R_{*}\left(0.139 R_{*}\right)$ & $0.044 R_{*}\left(0.139 R_{*}\right)$ & $0.044 R_{*}\left(0.139 R_{*}\right)$ & $0.044 R_{*}\left(0.139 R_{*}\right)$ & $0.044 R_{*}\left(0.139 R_{*}\right)$ & $0.052 R_{*}\left(0.167 R_{*}\right)$ & $0.052 R_{*}\left(0.167 R_{*}\right)$ & $0.052 R_{*}\left(0.167 R_{*}\right)$ \\
\hline 3625 & 150 & $0.052 R_{*}\left(0.167 R_{*}\right)$ & $0.052 R_{*}\left(0.167 R_{*}\right)$ & $0.052 R_{*}\left(0.167 R_{*}\right)$ & $0.052 R_{*}\left(0.167 R_{*}\right)$ & $0.052 R_{*}\left(0.167 R_{*}\right)$ & $0.061 R_{*}\left(0.194 R_{*}\right)$ & $0.061 R_{*}\left(0.194 R_{*}\right)$ & $0.061 R_{*}\left(0.194 R_{*}\right)$ & $0.070 R_{*}\left(0.222 R_{*}\right)$ \\
\hline 3625 & 200 & $0.052 R_{*}\left(0.167 R_{*}\right)$ & $0.052 R_{*}\left(0.167 R_{*}\right)$ & $0.061 R_{*}\left(0.194 R_{*}\right)$ & $0.061 R_{*}\left(0.194 R_{*}\right)$ & $0.070 R_{*}\left(0.222 R_{*}\right)$ & $0.070 R_{*}\left(0.222 R_{*}\right)$ & $0.079 R_{*}\left(0.250 R_{*}\right)$ & $0.079 R_{*}\left(0.250 R_{*}\right)$ & $0.079 R_{*}\left(0.250 R_{*}\right)$ \\
\hline
\end{tabular}

$R_{\mathrm{p}}=4.00 R_{\oplus}$

\begin{tabular}{|c|c|c|c|c|c|c|c|c|c|c|}
\hline 3400 & 60 & $0.017 R_{*}\left(0.056 R_{*}\right)$ & $0.017 R_{*}\left(0.056 R_{*}\right)$ & $0.017 R_{*}\left(0.056 R_{*}\right)$ & $0.017 R_{*}\left(0.056 R_{*}\right)$ & $0.017 R_{*}\left(0.056 R_{*}\right)$ & $0.017 R_{*}\left(0.056 R_{*}\right)$ & $0.026 R_{*}\left(0.083 R_{*}\right)$ & $0.026 R_{*}\left(0.083 R_{*}\right)$ & $0.026 R_{*}\left(0.083 R_{*}\right)$ \\
\hline 3400 & 100 & $0.026 R_{*}\left(0.083 R_{*}\right)$ & $0.026 R_{*}\left(0.083 R_{*}\right)$ & $0.026 R_{*}\left(0.083 R_{*}\right)$ & $0.026 R_{*}\left(0.083 R_{*}\right)$ & $0.026 R_{*}\left(0.083 R_{*}\right)$ & $0.026 R_{*}\left(0.083 R_{*}\right)$ & $0.026 R_{*}\left(0.083 R_{*}\right)$ & $0.026 R_{*}\left(0.083 R_{*}\right)$ & $0.026 R_{*}\left(0.083 R_{*}\right)$ \\
\hline 3400 & 150 & $26 R_{*}\left(0.083 R_{*}\right)$ & $0.026 R_{*}\left(0.083 R_{*}\right)$ & $0.026 R_{*}\left(0.083 R_{*}\right)$ & $026 R_{*}\left(0.083 R_{*}\right)$ & $0.026 R_{*}\left(0.083 R_{*}\right)$ & $0.026 R_{*}\left(0.083 R_{*}\right)$ & $0.035 R_{*}\left(0.111 R_{*}\right)$ & $0.035 R_{*}\left(0.111 R_{*}\right)$ & $0.035 R_{*}\left(0.111 R_{*}\right)$ \\
\hline 3400 & 200 & $026 R_{*}\left(0.083 R_{*}\right)$ & $0.035 R_{*}\left(0.111 R_{*}\right)$ & $0.035 R_{*}\left(0.111 R_{*}\right)$ & $0.035 R_{*}\left(0.111 R_{*}\right)$ & $0.035 R_{*}\left(0.111 R_{*}\right)$ & $0.035 R_{*}\left(0.111 R_{*}\right)$ & $0.035 R_{*}\left(0.111 R_{*}\right)$ & $0.035 R_{*}\left(0.111 R_{*}\right)$ & $0.035 R_{*}\left(0.111 R_{*}\right)$ \\
\hline 3475 & 60 & $017 R_{*}\left(0.056 R_{*}\right)$ & $0.017 R_{*}\left(0.056 R_{*}\right)$ & $0.017 R_{*}\left(0.056 R_{*}\right)$ & $0.026 R_{*}\left(0.083 R_{*}\right)$ & $0.026 R_{*}\left(0.083 R_{*}\right)$ & $0.026 R_{*}\left(0.083 R_{*}\right)$ & $0.026 R_{*}\left(0.083 R_{*}\right)$ & $0.026 R_{*}\left(0.083 R_{*}\right)$ & $\left..083 R_{*}\right)$ \\
\hline 3475 & 100 & $026 R_{*}\left(0.083 R_{*}\right)$ & $0.026 R_{*}\left(0.083 R_{*}\right)$ & $0.026 R_{*}\left(0.083 R_{*}\right)$ & $0.026 R_{*}\left(0.083 R_{*}\right)$ & $0.026 R_{*}\left(0.083 R_{*}\right)$ & $0.026 R_{*}\left(0.083 R_{*}\right)$ & $0.026 R_{*}\left(0.083 R_{*}\right)$ & $0.026 R_{*}\left(0.083 R_{*}\right)$ & $0.026 R_{*}\left(0.083 R_{*}\right)$ \\
\hline 3475 & 150 & $\left.111 R_{*}\right)$ & $0.026 R_{*}\left(0.083 R_{*}\right)$ & $0.026 R_{*}\left(0.083 R_{*}\right)$ & $\left.111 R_{*}\right)$ & $\mathrm{R}_{*}\left(0.111 R_{*}\right)$ & $\left.111 R_{*}\right)$ & $0.035 R_{*}\left(0.111 R_{*}\right)$ & $0.035 R_{*}\left(0.111 R_{*}\right)$ & $\left.0.111 R_{*}\right)$ \\
\hline 3475 & 200 & $0.035 R_{*}($ & $0.035 R_{*}(0$ & $0.035 R_{*}(0$ & $0.035 R_{*}\left(0.111 R_{*}\right)$ & $0.035 R_{*}\left(0.111 R_{*}\right)$ & $0.044 R_{*}\left(0.139 R_{*}\right)$ & $0.044 R_{*}\left(0.139 R_{*}\right)$ & $4 R_{*}\left(0.139 R_{*}\right)$ & $0.044 R_{*}\left(0.139 R_{*}\right)$ \\
\hline 3550 & 60 & $0.026 R_{*}\left(0.083 R_{*}\right)$ & $0.026 R_{*}\left(0.083 R_{*}\right)$ & $0.026 R_{*}\left(0.083 R_{*}\right)$ & $0.026 R_{*}\left(0.083 R_{*}\right)$ & $0.026 R_{*}\left(0.083 R_{*}\right)$ & $0.026 R_{*}\left(0.083 R_{*}\right)$ & $0.026 R_{*}\left(0.083 R_{*}\right)$ & $0.026 R_{*}\left(0.083 R_{*}\right)$ & $0.026 R_{*}\left(0.083 R_{*}\right)$ \\
\hline 3550 & 100 & $0.026 R_{*}\left(0.083 R_{*}\right)$ & $0.026 R_{*}\left(0.083 R_{*}\right)$ & $0.026 R_{*}\left(0.083 R_{*}\right)$ & $0.035 R_{*}\left(0.111 R_{*}\right)$ & $0.035 R_{*}\left(0.111 R_{*}\right)$ & $0.035 R_{*}\left(0.111 R_{*}\right)$ & $0.035 R_{*}\left(0.111 R_{*}\right)$ & $0.035 R_{*}\left(0.111 R_{*}\right)$ & $0.035 R_{*}\left(0.111 R_{*}\right)$ \\
\hline 3550 & 150 & $0.035 R_{*}\left(0.111 R_{*}\right)$ & $0.035 R_{*}\left(0.111 R_{*}\right)$ & $0.035 R_{*}\left(0.111 R_{*}\right)$ & $0.035 R_{*}\left(0.111 R_{*}\right)$ & $0.044 R_{*}\left(0.139 R_{*}\right)$ & $0.044 R_{*}\left(0.139 R_{*}\right)$ & $0.044 R_{*}\left(0.139 R_{*}\right)$ & $0.044 R_{*}\left(0.139 R_{*}\right)$ & $0.044 R_{*}\left(0.139 R_{*}\right)$ \\
\hline 3550 & 200 & $0.044 R_{*}\left(0.139 R_{*}\right)$ & $0.044 R_{*}\left(0.139 R_{*}\right)$ & $0.044 R_{*}\left(0.139 R_{*}\right)$ & $0.044 R_{*}\left(0.139 R_{*}\right)$ & $0.044 R_{*}\left(0.139 R_{*}\right)$ & $0.052 R_{*}\left(0.167 R_{*}\right)$ & $0.052 R_{*}\left(0.167 R_{*}\right)$ & $0.052 R_{*}\left(0.167 R_{*}\right)$ & $0.052 R_{*}\left(0.167 R_{*}\right)$ \\
\hline 3625 & 60 & $0.035 R_{*}\left(0.111 R_{*}\right)$ & $0.035 R_{*}\left(0.111 R_{*}\right)$ & $0.035 R_{*}\left(0.111 R_{*}\right)$ & $0.035 R_{*}\left(0.111 R_{*}\right)$ & $0.035 R_{*}\left(0.111 R_{*}\right)$ & $0.035 R_{*}\left(0.111 R_{*}\right)$ & $0.044 R_{*}\left(0.139 R_{*}\right)$ & $0.044 R_{*}\left(0.139 R_{*}\right)$ & $0.044 R_{*}\left(0.139 R_{*}\right)$ \\
\hline 3625 & 100 & $0.044 R_{*}\left(0.139 R_{*}\right)$ & $0.044 R_{*}\left(0.139 R_{*}\right)$ & $0.044 R_{*}\left(0.139 R_{*}\right)$ & $0.044 R_{*}\left(0.139 R_{*}\right)$ & $0.044 R_{*}\left(0.139 R_{*}\right)$ & $0.044 R_{*}\left(0.139 R_{*}\right)$ & $0.052 R_{*}\left(0.167 R_{*}\right)$ & $0.052 R_{*}\left(0.167 R_{*}\right)$ & $0.052 R_{*}\left(0.167 R_{*}\right)$ \\
\hline 3625 & 150 & $0.052 R_{*}\left(0.167 R_{*}\right)$ & $0.052 R_{*}\left(0.167 R_{*}\right)$ & $0.052 R_{*}\left(0.167 R_{*}\right)$ & $0.052 R_{*}\left(0.167 R_{*}\right)$ & $0.052 R_{*}\left(0.167 R_{*}\right)$ & $0.061 R_{*}\left(0.194 R_{*}\right)$ & $0.061 R_{*}\left(0.194 R_{*}\right)$ & $0.061 R_{*}\left(0.194 R_{*}\right)$ & $0.070 R_{*}\left(0.222 R_{*}\right)$ \\
\hline 3625 & 200 & $0.052 R_{*}\left(0.167 R_{*}\right)$ & $0.061 R_{*}\left(0.194 R_{*}\right)$ & $0.052 R_{*}\left(0.167 R_{*}\right)$ & $0.061 R_{*}\left(0.194 R_{*}\right)$ & $0.061 R_{*}\left(0.194 R_{*}\right)$ & $0.070 R_{*}\left(0.222 R_{*}\right)$ & $0.070 R_{*}\left(0.222 R_{*}\right)$ & $0.070 R_{*}\left(0.222 R_{*}\right)$ & $0.079 R_{*}\left(0.250 R_{*}\right)$ \\
\hline
\end{tabular}


Table A.3. Smallest detectable value of $r_{\text {spot }}$ determined from the 6737 simulations of the 1296 scenarios that used an K5V dwarf host star.

\begin{tabular}{|c|c|c|c|c|c|c|c|c|c|c|}
\hline \multirow{2}{*}{$\begin{array}{l}T_{\text {spot }} \\
(K)\end{array}$} & \multirow{2}{*}{$\begin{array}{c}\text { rms scatter } \\
\text { (ppm) }\end{array}$} & \multicolumn{3}{|c|}{$\begin{array}{c}\lambda_{\mathrm{obs}}=600 \mathrm{~nm} \\
P\end{array}$} & \multicolumn{3}{|c|}{$\begin{array}{c}\lambda_{\mathrm{obs}}=785 \mathrm{~nm} \\
P\end{array}$} & \multicolumn{3}{|c|}{$\begin{array}{c}\lambda_{\mathrm{obs}}=1000 \mathrm{~nm} \\
P\end{array}$} \\
\hline & & $2 \mathrm{~d}$ & $4 \mathrm{~d}$ & $6 \mathrm{~d}$ & $2 \mathrm{~d}$ & $4 \mathrm{~d}$ & $6 \mathrm{~d}$ & $2 \mathrm{~d}$ & $4 \mathrm{~d}$ & $6 \mathrm{~d}$ \\
\hline \multicolumn{11}{|c|}{$R_{\mathrm{p}}=3.00 R_{\oplus}$} \\
\hline 3700 & 60 & $0.017 R_{*}\left(0.070 R_{*}\right)$ & $0.017 R_{*}\left(0.070 R_{*}\right)$ & $0.026 R_{*}\left(0.105 R_{*}\right)$ & $0.017 R_{*}\left(0.070 R_{*}\right)$ & $0.017 R_{*}\left(0.070 R_{*}\right)$ & $0.026 R_{*}\left(0.105 R_{*}\right)$ & $0.017 R_{*}\left(0.070 R_{*}\right)$ & $0.026 R_{*}\left(0.105 R_{*}\right)$ & $0.026 R_{*}\left(0.105 R_{*}\right)$ \\
\hline 3700 & 100 & $0.026 R_{*}\left(0.105 R_{*}\right)$ & $0.026 R_{*}\left(0.105 R_{*}\right)$ & $0.026 R_{*}\left(0.105 R_{*}\right)$ & $0.026 R_{*}\left(0.105 R_{*}\right)$ & $0.026 R_{*}\left(0.105 R_{*}\right)$ & $0.035 R_{*}\left(0.140 R_{*}\right)$ & $0.026 R_{*}\left(0.105 R_{*}\right)$ & $0.035 R_{*}\left(0.140 R_{*}\right)$ & $0.035 R_{*}\left(0.140 R_{*}\right)$ \\
\hline 3700 & 150 & $0.026 R_{*}\left(0.105 R_{*}\right)$ & $0.035 R_{*}\left(0.140 R_{*}\right)$ & $0.035 R_{*}\left(0.140 R_{*}\right)$ & $0.026 R_{*}\left(0.105 R_{*}\right)$ & $0.035 R_{*}\left(0.140 R_{*}\right)$ & $0.035 R_{*}\left(0.140 R_{*}\right)$ & $0.035 R_{*}\left(0.140 R_{*}\right)$ & $0.035 R_{*}\left(0.140 R_{*}\right)$ & $0.044 R_{*}\left(0.175 R_{*}\right)$ \\
\hline 3700 & 200 & $0.035 R_{*}\left(0.140 R_{*}\right)$ & $0.035 R_{*}\left(0.140 R_{*}\right)$ & $0.044 R_{*}\left(0.175 R_{*}\right)$ & $0.035 R_{*}\left(0.140 R_{*}\right)$ & $0.035 R_{*}\left(0.140 R_{*}\right)$ & $0.044 R_{*}\left(0.175 R_{*}\right)$ & $0.035 R_{*}\left(0.140 R_{*}\right)$ & $0.044 R_{*}\left(0.175 R_{*}\right)$ & $0.044 R_{*}\left(0.175 R_{*}\right)$ \\
\hline 3800 & 60 & $0.017 R_{*}\left(0.070 R_{*}\right)$ & $0.017 R_{*}\left(0.070 R_{*}\right)$ & $0.026 R_{*}\left(0.105 R_{*}\right)$ & $0.017 R_{*}\left(0.070 R_{*}\right)$ & $0.026 R_{*}\left(0.105 R_{*}\right)$ & $0.026 R_{*}\left(0.105 R_{*}\right)$ & $0.026 R_{*}\left(0.105 R_{*}\right)$ & $0.026 R_{*}\left(0.105 R_{*}\right)$ & $0.026 R_{*}\left(0.105 R_{*}\right)$ \\
\hline 3800 & 100 & $0.026 R_{*}\left(0.105 R_{*}\right)$ & $0.026 R_{*}\left(0.105 R_{*}\right)$ & $0.035 R_{*}\left(0.140 R_{*}\right)$ & $0.026 R_{*}\left(0.105 R_{*}\right)$ & $0.035 R_{*}\left(0.140 R_{*}\right)$ & $0.035 R_{*}\left(0.140 R_{*}\right)$ & $0.026 R_{*}\left(0.105 R_{*}\right)$ & $0.035 R_{*}\left(0.140 R_{*}\right)$ & $0.044 R_{*}\left(0.175 R_{*}\right)$ \\
\hline 3800 & 150 & $0.026 R_{*}\left(0.105 R_{*}\right)$ & $0.035 R_{*}\left(0.140 R_{*}\right)$ & $0.035 R_{*}\left(0.140 R_{*}\right)$ & $0.035 R_{*}\left(0.140 R_{*}\right)$ & $0.044 R_{*}\left(0.175 R_{*}\right)$ & $0.044 R_{*}\left(0.175 R_{*}\right)$ & $0.035 R_{*}\left(0.140 R_{*}\right)$ & $0.044 R_{*}\left(0.175 R_{*}\right)$ & $0.052 R_{*}\left(0.209 R_{*}\right)$ \\
\hline 3800 & 200 & $0.035 R_{*}\left(0.140 R_{*}\right)$ & $0.035 R_{*}\left(0.140 R_{*}\right)$ & $0.044 R_{*}\left(0.175 R_{*}\right)$ & $0.044 R_{*}\left(0.175 R_{*}\right)$ & $0.044 R_{*}\left(0.175 R_{*}\right)$ & $0.052 R_{*}\left(0.209 R_{*}\right)$ & $0.044 R_{*}\left(0.175 R_{*}\right)$ & $0.052 R_{*}\left(0.209 R_{*}\right)$ & $0.061 R_{*}\left(0.244 R_{*}\right)$ \\
\hline 3900 & 60 & $0.017 R_{*}\left(0.070 R_{*}\right)$ & $0.026 R_{*}\left(0.105 R_{*}\right)$ & $0.026 R_{*}\left(0.105 R_{*}\right)$ & $0.026 R_{*}\left(0.105 R_{*}\right)$ & $0.026 R_{*}\left(0.105 R_{*}\right)$ & $0.035 R_{*}\left(0.140 R_{*}\right)$ & $0.026 R_{*}\left(0.105 R_{*}\right)$ & $0.026 R_{*}\left(0.105 R_{*}\right)$ & $0.035 R_{*}\left(0.140 R_{*}\right)$ \\
\hline 3900 & 100 & $0.026 R_{*}\left(0.105 R_{*}\right)$ & $0.035 R_{*}\left(0.140 R_{*}\right)$ & $0.035 R_{*}\left(0.140 R_{*}\right)$ & $0.035 R_{*}\left(0.140 R_{*}\right)$ & $0.035 R_{*}\left(0.140 R_{*}\right)$ & $0.044 R_{*}\left(0.175 R_{*}\right)$ & $0.035 R_{*}\left(0.140 R_{*}\right)$ & $0.044 R_{*}\left(0.175 R_{*}\right)$ & $0.052 R_{*}\left(0.209 R_{*}\right)$ \\
\hline 3900 & 150 & $0.035 R_{*}\left(0.140 R_{*}\right)$ & $0.044 R_{*}\left(0.175 R_{*}\right)$ & $0.044 R_{*}\left(0.175 R_{*}\right)$ & $0.044 R_{*}\left(0.175 R_{*}\right)$ & $0.044 R_{*}\left(0.175 R_{*}\right)$ & $0.061 R_{*}\left(0.244 R_{*}\right)$ & $0.044 R_{*}\left(0.175 R_{*}\right)$ & $0.061 R_{*}\left(0.244 R_{*}\right)$ & $0.070 R_{*}\left(0.279 R_{*}\right)$ \\
\hline 3900 & 200 & $0.044 R_{*}\left(0.175 R_{*}\right)$ & $0.052 R_{*}\left(0.209 R_{*}\right)$ & $0.061 R_{*}\left(0.244 R_{*}\right)$ & $0.052 R_{*}\left(0.209 R_{*}\right)$ & $0.061 R_{*}\left(0.244 R_{*}\right)$ & $0.079 R_{*}\left(0.314 R_{*}\right)$ & $0.061 R_{*}\left(0.244 R_{*}\right)$ & $0.079 R_{*}\left(0.314 R_{*}\right)$ & $0.087 R_{*}\left(0.349 R_{*}\right)$ \\
\hline 4000 & 60 & $0.026 R_{*}\left(0.105 R_{*}\right)$ & $0.035 R_{*}\left(0.140 R_{*}\right)$ & $0.035 R_{*}\left(0.140 R_{*}\right)$ & $0.035 R_{*}\left(0.140 R_{*}\right)$ & $0.035 R_{*}\left(0.140 R_{*}\right)$ & $0.044 R_{*}\left(0.175 R_{*}\right)$ & $0.035 R_{*}\left(0.140 R_{*}\right)$ & $0.044 R_{*}\left(0.175 R_{*}\right)$ & $0.044 R_{*}\left(0.175 R_{*}\right)$ \\
\hline 4000 & 100 & $0.044 R_{*}\left(0.175 R_{*}\right)$ & $0.044 R_{*}\left(0.175 R_{*}\right)$ & $0.052 R_{*}\left(0.209 R_{*}\right)$ & $0.044 R_{*}\left(0.175 R_{*}\right)$ & $0.061 R_{*}\left(0.244 R_{*}\right)$ & $0.070 R_{*}\left(0.279 R_{*}\right)$ & $0.061 R_{*}\left(0.244 R_{*}\right)$ & $0.079 R_{*}\left(0.314 R_{*}\right)$ & $0.096 R_{*}\left(0.384 R_{*}\right)$ \\
\hline 4000 & 150 & $0.052 R_{*}\left(0.209 R_{*}\right)$ & $0.070 R_{*}\left(0.279 R_{*}\right)$ & $0.079 R_{*}\left(0.314 R_{*}\right)$ & $0.183 R_{*}\left(0.733 R_{*}\right)$ & $0.140 R_{*}\left(0.559 R_{*}\right)$ & $0.271 R_{*}\left(1.082 R_{*}\right)$ & $\mathrm{NaN}$ & $\mathrm{NaN}$ & $\mathrm{NaN}$ \\
\hline 4000 & 200 & $\mathrm{NaN}$ & $\mathrm{NaN}$ & $\mathrm{NaN}$ & $\mathrm{NaN}$ & $\mathrm{NaN}$ & $\mathrm{NaN}$ & $\mathrm{NaN}$ & $\mathrm{NaN}$ & $\mathrm{NaN}$ \\
\hline
\end{tabular}
$R_{\mathrm{p}}=3.50 R_{\oplus}$

\begin{tabular}{|c|c|c|c|c|c|c|c|c|c|c|}
\hline 3700 & 60 & $0.017 R_{*}\left(0.070 R_{*}\right)$ & $0.017 R_{*}\left(0.070 R_{*}\right)$ & $0.017 R_{*}\left(0.070 R_{*}\right)$ & $0.017 R_{*}\left(0.070 R_{*}\right)$ & $0.017 R_{*}\left(0.070 R_{*}\right)$ & $0.026 R_{*}\left(0.105 R_{*}\right)$ & $0.017 R_{*}\left(0.070 R_{*}\right)$ & $0.017 R_{*}\left(0.070 R_{*}\right)$ & $0.026 R_{*}\left(0.105 R_{*}\right)$ \\
\hline 3700 & 100 & $0.017 R_{*}\left(0.070 R_{*}\right)$ & $0.026 R_{*}\left(0.105 R_{*}\right)$ & $026 R_{*}\left(0.105 R_{*}\right)$ & $.026 R_{*}\left(0.105 R_{*}\right)$ & $0.026 R_{*}\left(0.105 R_{*}\right)$ & $0.026 R_{*}\left(0.105 R_{*}\right)$ & $0.026 R_{*}\left(0.105 R_{*}\right)$ & $0.026 R_{*}\left(0.105 R_{*}\right)$ & $0.035 R_{*}\left(0.140 R_{*}\right)$ \\
\hline 3700 & 150 & $0.026 R_{*}\left(0.105 R_{*}\right)$ & $0.026 R_{*}\left(0.105 R_{*}\right)$ & $0.035 R_{*}\left(0.140 R_{*}\right)$ & $.026 R_{*}\left(0.105 R_{*}\right)$ & $0.035 R_{*}\left(0.140 R_{*}\right)$ & $0.035 R_{*}\left(0.140 R_{*}\right)$ & $0.035 R_{*}\left(0.140 R_{*}\right)$ & $0.035 R_{*}\left(0.140 R_{*}\right)$ & $0.035 R_{*}\left(0.140 R_{*}\right)$ \\
\hline 3700 & 200 & $0.035 R_{*}\left(0.140 R_{*}\right)$ & $0.035 R_{*}\left(0.140 R_{*}\right)$ & $0.035 R_{*}\left(0.140 R_{*}\right)$ & $0.035 R_{*}\left(0.140 R_{*}\right)$ & $0.035 R_{*}\left(0.140 R_{*}\right)$ & $0.044 R_{*}\left(0.175 R_{*}\right)$ & $0.035 R_{*}\left(0.140 R_{*}\right)$ & $0.044 R_{*}\left(0.175 R_{*}\right)$ & $0.044 R_{*}\left(0.175 R_{*}\right)$ \\
\hline 3800 & 60 & $0.017 R_{*}\left(0.070 R_{*}\right)$ & $0.017 R_{*}\left(0.070 R_{*}\right)$ & $0.026 R_{*}\left(0.105 R_{*}\right)$ & $0.017 R_{*}\left(0.070 R_{*}\right)$ & $0.017 R_{*}\left(0.070 R_{*}\right)$ & $0.026 R_{*}\left(0.105 R_{*}\right)$ & $0.017 R_{*}\left(0.070 R_{*}\right)$ & $0.026 R_{*}\left(0.105 R_{*}\right)$ & $0.026 R_{*}\left(0.105 R_{*}\right)$ \\
\hline 3800 & 100 & $0.026 R_{*}\left(0.105 R_{*}\right)$ & $0.026 R_{*}\left(0.105 R_{*}\right)$ & $0.026 R_{*}\left(0.105 R_{*}\right)$ & $0.026 R_{*}\left(0.105 R_{*}\right)$ & $0.026 R_{*}\left(0.105 R_{*}\right)$ & $0.035 R_{*}\left(0.140 R_{*}\right)$ & $0.026 R_{*}\left(0.105 R_{*}\right)$ & $0.035 R_{*}\left(0.140 R_{*}\right)$ & $0.035 R_{*}\left(0.140 R_{*}\right)$ \\
\hline 3800 & 150 & $0.026 R_{*}\left(0.105 R_{*}\right)$ & $0.035 R_{*}\left(0.140 R_{*}\right)$ & $0.035 R_{*}\left(0.140 R_{*}\right)$ & $0.035 R_{*}\left(0.140 R_{*}\right)$ & $0.035 R_{*}\left(0.140 R_{*}\right)$ & $0.044 R_{*}\left(0.175 R_{*}\right)$ & $0.035 R_{*}\left(0.140 R_{*}\right)$ & $0.044 R_{*}\left(0.175 R_{*}\right)$ & $0.044 R_{*}\left(0.175 R_{*}\right)$ \\
\hline 3800 & 200 & $0.035 R_{*}\left(0.140 R_{*}\right)$ & $0.035 R_{*}\left(0.140 R_{*}\right)$ & $0.044 R_{*}\left(0.175 R_{*}\right)$ & $0.035 R_{*}\left(0.140 R_{*}\right)$ & $0.044 R_{*}\left(0.175 R_{*}\right)$ & $0.044 R_{*}\left(0.175 R_{*}\right)$ & $0.044 R_{*}\left(0.175 R_{*}\right)$ & $0.044 R_{*}\left(0.175 R_{*}\right)$ & $0.052 R_{*}\left(0.209 R_{*}\right)$ \\
\hline 3900 & 60 & $017 R_{*}\left(0.070 R_{*}\right)$ & $0.026 R_{*}\left(0.105 R_{*}\right)$ & $0.026 R_{*}\left(0.105 R_{*}\right)$ & $0.026 R_{*}\left(0.105 R_{*}\right)$ & $0.026 R_{*}\left(0.105 R_{*}\right)$ & $0.026 R_{*}\left(0.105 R_{*}\right)$ & $0.026 R_{*}\left(0.105 R_{*}\right)$ & $0.026 R_{*}\left(0.105 R_{*}\right)$ & $0.035 R_{*}\left(0.140 R_{*}\right)$ \\
\hline 3900 & 100 & $0.026 R_{*}\left(0.105 R_{*}\right)$ & $0.035 R_{*}\left(0.140 R_{*}\right)$ & $0.035 R_{*}\left(0.140 R_{*}\right)$ & $0.035 R_{*}\left(0.140 R_{*}\right)$ & $0.035 R_{*}\left(0.140 R_{*}\right)$ & $0.044 R_{*}\left(0.175 R_{*}\right)$ & $0.035 R_{*}\left(0.140 R_{*}\right)$ & $0.035 R_{*}\left(0.140 R_{*}\right)$ & $0.044 R_{*}\left(0.175 R_{*}\right)$ \\
\hline 3900 & 150 & $0.035 R_{*}\left(0.140 R_{*}\right)$ & $0.035 R_{*}\left(0.140 R_{*}\right)$ & $0.044 R_{*}\left(0.175 R_{*}\right)$ & $0.035 R_{*}\left(0.140 R_{*}\right)$ & $0.044 R_{*}\left(0.175 R_{*}\right)$ & $0.052 R_{*}\left(0.209 R_{*}\right)$ & $0.044 R_{*}\left(0.175 R_{*}\right)$ & $0.052 R_{*}\left(0.209 R_{*}\right)$ & $0.061 R_{*}\left(0.244 R_{*}\right)$ \\
\hline 3900 & 200 & $0.035 R_{*}\left(0.140 R_{*}\right)$ & $0.044 R_{*}\left(0.175 R_{*}\right)$ & $0.052 R_{*}\left(0.209 R_{*}\right)$ & $0.044 R_{*}\left(0.175 R_{*}\right)$ & $0.052 R_{*}\left(0.209 R_{*}\right)$ & $0.061 R_{*}\left(0.244 R_{*}\right)$ & $0.052 R_{*}\left(0.209 R_{*}\right)$ & $0.052 R_{*}\left(0.209 R_{*}\right)$ & $0.070 R_{*}\left(0.279 R_{*}\right)$ \\
\hline 4000 & 60 & $0.026 R_{*}\left(0.105 R_{*}\right)$ & $0.026 R_{*}\left(0.105 R_{*}\right)$ & $0.035 R_{*}\left(0.140 R_{*}\right)$ & $0.035 R_{*}\left(0.140 R_{*}\right)$ & $0.035 R_{*}\left(0.140 R_{*}\right)$ & $0.035 R_{*}\left(0.140 R_{*}\right)$ & $0.035 R_{*}\left(0.140 R_{*}\right)$ & $0.035 R_{*}\left(0.140 R_{*}\right)$ & $0.044 R_{*}\left(0.175 R_{*}\right)$ \\
\hline 4000 & 100 & $0.035 R_{*}\left(0.140 R_{*}\right)$ & $0.044 R_{*}\left(0.175 R_{*}\right)$ & $0.052 R_{*}\left(0.209 R_{*}\right)$ & $0.044 R_{*}\left(0.175 R_{*}\right)$ & $0.052 R_{*}\left(0.209 R_{*}\right)$ & $0.052 R_{*}\left(0.209 R_{*}\right)$ & $0.052 R_{*}\left(0.209 R_{*}\right)$ & $0.052 R_{*}\left(0.209 R_{*}\right)$ & $0.061 R_{*}\left(0.244 R_{*}\right)$ \\
\hline 4000 & 150 & $0.052 R_{*}\left(0.209 R_{*}\right)$ & $0.052 R_{*}\left(0.209 R_{*}\right)$ & $0.061 R_{*}\left(0.244 R_{*}\right)$ & $0.061 R_{*}\left(0.244 R_{*}\right)$ & $0.070 R_{*}\left(0.279 R_{*}\right)$ & $0.079 R_{*}\left(0.314 R_{*}\right)$ & $0.070 R_{*}\left(0.279 R_{*}\right)$ & $0.087 R_{*}\left(0.349 R_{*}\right)$ & $0.113 R_{*}\left(0.454 R_{*}\right)$ \\
\hline 4000 & 200 & $0.061 R_{*}\left(0.244 R_{*}\right)$ & $0.070 R_{*}\left(0.279 R_{*}\right)$ & $0.087 R_{*}\left(0.349 R_{*}\right)$ & $0.175 R_{*}\left(0.698 R_{*}\right)$ & $0.096 R_{*}\left(0.384 R_{*}\right)$ & $0.122 R_{*}\left(0.489 R_{*}\right)$ & $\mathrm{NaN}$ & $\mathrm{NaN}$ & $\mathrm{NaN}$ \\
\hline
\end{tabular}

$R_{\mathrm{p}}=4.00 R_{\oplus}$

\begin{tabular}{|c|c|c|c|c|c|c|c|c|c|c|}
\hline 3700 & 60 & $0.017 R_{*}\left(0.070 R_{*}\right)$ & $0.017 R_{*}\left(0.070 R_{*}\right)$ & $0.017 R_{*}\left(0.070 R_{*}\right)$ & $0.017 R_{*}\left(0.070 R_{*}\right)$ & $0.017 R_{*}\left(0.070 R_{*}\right)$ & $0.017 R_{*}\left(0.070 R_{*}\right)$ & $0.017 R_{*}\left(0.070 R_{*}\right)$ & $0.017 R_{*}\left(0.070 R_{*}\right)$ & $\left.105 R_{*}\right)$ \\
\hline 3700 & 100 & $026 R_{*}\left(0.105 R_{*}\right)$ & $\left(0.105 R_{*}\right)$ & $26 R_{*}\left(0.105 R_{*}\right)$ & $026 R_{*}\left(0.105 R_{*}\right)$ & $0.026 R_{*}\left(0.105 R_{*}\right)$ & $0.026 R_{*}\left(0.105 R_{*}\right)$ & $0.026 R_{*}\left(0.105 R_{*}\right)$ & $0.026 R$ & $\left.05 R_{*}\right)$ \\
\hline 3700 & 150 & $0.026 R_{*}\left(0.105 R_{*}\right)$ & $0.026 R_{*}\left(0.105 R_{*}\right)$ & $0.026 R_{*}\left(0.105 R_{*}\right)$ & $026 R_{*}\left(0.105 R_{*}\right)$ & $0.026 R_{*}\left(0.105 R_{*}\right)$ & $0.035 R_{*}\left(0.140 R_{*}\right)$ & $.026 R_{*}\left(0.105 R_{*}\right)$ & $0.035 R_{*}\left(0.140 R_{*}\right)$ & $0.035 R_{*}\left(0.140 R_{*}\right)$ \\
\hline 3700 & 200 & $0.026 R_{*}\left(0.105 R_{*}\right)$ & $0.035 R_{*}\left(0.140 R_{*}\right)$ & $0.035 R_{*}\left(0.140 R_{*}\right)$ & $0.035 R_{*}\left(0.140 R_{*}\right)$ & $0.035 R_{*}\left(0.140 R_{*}\right)$ & $0.035 R_{*}\left(0.140 R_{*}\right)$ & $0.035 R_{*}\left(0.140 R_{*}\right)$ & $0.035 R_{*}\left(0.140 R_{*}\right)$ & $0.044 R_{*}\left(0.175 R_{*}\right)$ \\
\hline 3800 & 60 & $0.017 R_{*}\left(0.070 R_{*}\right)$ & $0.017 R_{*}\left(0.070 R_{*}\right)$ & $0.017 R_{*}\left(0.070 R_{*}\right)$ & $0.017 R_{*}\left(0.070 R_{*}\right)$ & $0.017 R_{*}\left(0.070 R_{*}\right)$ & $0.026 R_{*}\left(0.105 R_{*}\right)$ & $0.017 R_{*}\left(0.070 R_{*}\right)$ & $0.026 R_{*}\left(0.105 R_{*}\right)$ & $0.026 R_{*}\left(0.105 R_{*}\right)$ \\
\hline 3800 & 100 & $0.026 R_{*}\left(0.105 R_{*}\right)$ & $0.026 R_{*}\left(0.105 R_{*}\right)$ & $.026 R_{*}\left(0.105 R_{*}\right)$ & $0.026 R_{*}\left(0.105 R_{*}\right)$ & $0.026 R_{*}\left(0.105 R_{*}\right)$ & $0.035 R_{* *}\left(0.140 R_{*}\right)$ & $0.026 R_{*}\left(0.105 R_{*}\right)$ & $0.026 R_{*}\left(0.105 R_{*}\right)$ & $0.035 R_{*}\left(0.140 R_{*}\right)$ \\
\hline 3800 & 150 & $0.026 R_{*}\left(0.105 R_{*}\right)$ & $0.026 R_{*}\left(0.105 R_{*}\right)$ & $0.035 R_{*}\left(0.140 R_{*}\right)$ & $0.035 R_{*}\left(0.140 R_{*}\right)$ & $0.035 R_{*}\left(0.140 R_{*}\right)$ & $0.035 R_{*}\left(0.140 R_{*}\right)$ & $0.035 R_{*}\left(0.140 R_{*}\right)$ & $0.035 R_{*}\left(0.140 R_{*}\right)$ & $0.044 R_{*}\left(0.175 R_{*}\right)$ \\
\hline 3800 & 200 & $0.035 R_{*}\left(0.140 R_{*}\right)$ & $0.035 R_{*}\left(0.140 R_{*}\right)$ & $0.035 R_{*}\left(0.140 R_{*}\right)$ & $0.035 R_{*}\left(0.140 R_{*}\right)$ & $0.035 R_{*}\left(0.140 R_{*}\right)$ & $0.044 R_{*}\left(0.175 R_{*}\right)$ & $0.035 R_{*}\left(0.140 R_{*}\right)$ & $0.044 R_{*}\left(0.175 R_{*}\right)$ & $0.044 R_{*}\left(0.175 R_{*}\right)$ \\
\hline 3900 & 60 & $017 R_{*}\left(0.070 R_{*}\right)$ & $\left.0.105 R_{*}\right)$ & $0.026 R_{*}\left(0.105 R_{*}\right)$ & $0.026 R_{*}\left(0.105 R_{*}\right)$ & $0.026 R_{*}\left(0.105 R_{*}\right)$ & $0.026 R_{*}\left(0.105 R_{*}\right)$ & $0.026 R_{*}\left(0.105 R_{*}\right)$ & $0.026 R_{*}\left(0.105 R_{*}\right)$ & $0.026 R_{*}\left(0.105 R_{*}\right)$ \\
\hline 3900 & 100 & $0.026 R_{*}\left(0.105 R_{*}\right)$ & $0.026 R_{*}\left(0.105 R_{*}\right)$ & $0.035 R_{*}\left(0.140 R_{*}\right)$ & $0.026 R_{*}\left(0.105 R_{*}\right)$ & $0.035 R_{*}\left(0.140 R_{*}\right)$ & $0.035 R_{*}\left(0.140 R_{*}\right)$ & $0.035 R_{*}\left(0.140 R_{*}\right)$ & $0.035 R_{*}\left(0.140 R_{*}\right)$ & $0.044 R_{*}\left(0.175 R_{*}\right)$ \\
\hline 3900 & 150 & $0.035 R_{*}\left(0.140 R_{*}\right)$ & $0.035 R_{*}\left(0.140 R_{*}\right)$ & $0.044 R_{*}\left(0.175 R_{*}\right)$ & $0.035 R_{*}\left(0.140 R_{*}\right)$ & $0.044 R_{*}\left(0.175 R_{*}\right)$ & $0.044 R_{*}$ & $\left.0.175 R_{*}\right)$ & $\left(0.175 R_{*}\right)$ & $\left(0.209 R_{*}\right)$ \\
\hline 3900 & 200 & $035 R_{*}\left(0.140 R_{*}\right)$ & $0.044 R_{*}\left(0.175 R_{*}\right)$ & $0.044 R_{*}\left(0.175 R_{*}\right)$ & $0.044 R_{*}\left(0.175 R_{*}\right)$ & $0.052 R_{*}\left(0.209 R_{*}\right)$ & $0.052 R_{*}\left(0.209 R_{*}\right)$ & $0.044 R_{*}\left(0.175 R_{*}\right)$ & $0.052 R_{*}\left(0.209 R_{*}\right)$ & $0.061 R_{*}\left(0.244 R_{*}\right)$ \\
\hline 4000 & 60 & $0.026 R_{*}\left(0.105 R_{*}\right)$ & $0.026 R_{*}\left(0.105 R_{*}\right)$ & $0.035 R_{*}\left(0.140 R_{*}\right)$ & $0.026 R_{*}\left(0.105 R_{*}\right)$ & $0.035 R_{*}\left(0.140 R_{*}\right)$ & $0.035 R_{*}\left(0.140 R_{*}\right)$ & $0.035 R_{*}\left(0.140 R_{*}\right)$ & $0.035 R_{*}\left(0.140 R_{*}\right)$ & $0.044 R_{*}\left(0.175 R_{*}\right)$ \\
\hline 4000 & 100 & $0.035 R_{*}\left(0.140 R_{*}\right)$ & $0.044 R_{*}\left(0.175 R_{*}\right)$ & $0.044 R_{*}\left(0.175 R_{*}\right)$ & $0.044 R_{*}\left(0.175 R_{*}\right)$ & $0.044 R_{*}\left(0.175 R_{*}\right)$ & $0.044 R_{*}\left(0.175 R_{*}\right)$ & $0.052 R_{*}\left(0.209 R_{*}\right)$ & $0.052 R_{*}\left(0.209 R_{*}\right)$ & $0.061 R_{*}\left(0.244 R_{*}\right)$ \\
\hline 4000 & 150 & $0.044 R_{*}\left(0.175 R_{*}\right)$ & $0.052 R_{*}\left(0.209 R_{*}\right)$ & $0.052 R_{*}\left(0.209 R_{*}\right)$ & $0.052 R_{*}\left(0.209 R_{*}\right)$ & $0.061 R_{*}\left(0.244 R_{*}\right)$ & $0.061 R_{*}\left(0.244 R_{*}\right)$ & $0.061 R_{*}\left(0.244 R_{*}\right)$ & $0.070 R_{*}\left(0.279 R_{*}\right)$ & $0.079 R_{*}\left(0.314 R_{*}\right)$ \\
\hline 4000 & 200 & $0.052 R_{*}\left(0.209 R_{*}\right)$ & $0.061 R_{*}\left(0.244 R_{*}\right)$ & $0.061 R_{*}\left(0.244 R_{*}\right)$ & $0.061 R_{*}\left(0.244 R_{*}\right)$ & $0.070 R_{*}\left(0.279 R_{*}\right)$ & $0.087 R_{*}\left(0.349 R_{*}\right)$ & $0.105 R_{*}\left(0.419 R_{*}\right)$ & $0.105 R_{*}\left(0.419 R_{*}\right)$ & $0.113 R_{*}\left(0.454 R_{*}\right)$ \\
\hline
\end{tabular}

$R_{\mathrm{p}}=4.50 R_{\oplus}$

\begin{tabular}{|c|c|c|c|c|c|c|c|c|c|c|}
\hline 3700 & 60 & $0.017 R_{*}\left(0.070 R_{*}\right)$ & $0.017 R_{*}\left(0.070 R_{*}\right)$ & $0.017 R_{*}\left(0.070 R_{*}\right)$ & $0.017 R_{*}\left(0.070 R_{*}\right)$ & $0.017 R_{*}\left(0.070 R_{*}\right)$ & $0.017 R_{*}\left(0.070 R_{*}\right)$ & $0.017 R_{*}\left(0.070 R_{*}\right)$ & $0.017 R_{*}\left(0.070 R_{*}\right)$ & \\
\hline 3700 & 100 & $0.026 R_{*}\left(0.105 R_{*}\right)$ & $0.026 R_{*}\left(0.105 R_{*}\right)$ & $0.026 R_{*}\left(0.105 R_{*}\right)$ & $0.026 R_{*}\left(0.105 R_{*}\right)$ & $0.026 R_{*}\left(0.105 R_{*}\right)$ & $0.026 R_{*}\left(0.105 R_{*}\right)$ & $0.026 R_{*}\left(0.105 R_{*}\right)$ & $0.026 R_{*}\left(0.105 R_{*}\right)$ & $0.026 R_{*}\left(0.105 R_{*}\right)$ \\
\hline 3700 & 150 & $0.026 R_{*}\left(0.105 R_{*}\right)$ & $0.026 R_{*}\left(0.105 R_{*}\right)$ & $0.026 R_{*}\left(0.105 R_{*}\right)$ & $0.026 R_{*}\left(0.105 R_{*}\right)$ & $0.026 R_{*}\left(0.105 R_{*}\right)$ & $0.035 R_{*}\left(0.140 R_{*}\right)$ & $0.026 R_{*}\left(0.105 R_{*}\right)$ & $0.035 R_{*}\left(0.140 R_{*}\right)$ & $0.035 R_{*}\left(0.140 R_{*}\right)$ \\
\hline 3700 & 200 & $0.026 R_{*}\left(0.105 R_{*}\right)$ & $0.026 R_{*}\left(0.105 R_{*}\right)$ & $0.035 R_{*}\left(0.140 R_{*}\right)$ & $0.035 R_{*}\left(0.140 R_{*}\right)$ & $0.035 R_{*}\left(0.140 R_{*}\right)$ & $0.035 R_{*}\left(0.140 R_{*}\right)$ & $0.035 R_{*}\left(0.140 R_{*}\right)$ & $0.035 R_{*}\left(0.140 R_{*}\right)$ & $0.035 R_{*}\left(0.140 R_{*}\right)$ \\
\hline 3800 & 60 & $0.017 R_{*}\left(0.070 R_{*}\right)$ & $0.017 R_{*}\left(0.070 R_{*}\right)$ & $0.017 R_{*}\left(0.070 R_{*}\right)$ & $0.017 R_{*}\left(0.070 R_{*}\right)$ & $0.017 R_{*}\left(0.070 R_{*}\right)$ & $0.026 R_{*}\left(0.105 R_{*}\right)$ & $0.026 R_{*}\left(0.105 R_{*}\right)$ & $0.017 R_{*}\left(0.070 R_{*}\right)$ & $0.026 R_{*}\left(0.105 R_{*}\right)$ \\
\hline 3800 & 100 & $0.026 R_{*}\left(0.105 R_{*}\right)$ & $0.026 R_{*}\left(0.105 R_{*}\right)$ & $0.026 R_{*}\left(0.105 R_{*}\right)$ & $0.026 R_{*}\left(0.105 R_{*}\right)$ & $0.026 R_{*}\left(0.105 R_{*}\right)$ & $0.026 R_{*}\left(0.105 R_{*}\right)$ & $0.026 R_{*}\left(0.105 R_{*}\right)$ & $0.026 R_{*}\left(0.105 R_{*}\right)$ & $0.035 R_{*}\left(0.140 R_{*}\right)$ \\
\hline 3800 & 150 & $0.026 R_{*}\left(0.105 R_{*}\right)$ & $0.026 R_{*}\left(0.105 R_{*}\right)$ & $0.035 R_{*}\left(0.140 R_{*}\right)$ & $0.035 R_{*}\left(0.140 R_{*}\right)$ & $0.035 R_{*}\left(0.140 R_{*}\right)$ & $0.035 R_{*}\left(0.140 R_{*}\right)$ & $0.035 R_{*}\left(0.140 R_{*}\right)$ & $0.035 R_{*}\left(0.140 R_{*}\right)$ & $0.035 R_{*}\left(0.140 R_{*}\right)$ \\
\hline 3800 & 200 & $0.035 R_{*}\left(0.140 R_{*}\right)$ & $0.035 R_{*}\left(0.140 R_{*}\right)$ & $0.035 R_{*}\left(0.140 R_{*}\right)$ & $0.035 R_{*}\left(0.140 R_{*}\right)$ & $0.035 R_{*}\left(0.140 R_{*}\right)$ & $0.044 R_{*}\left(0.175 R_{*}\right)$ & $0.044 R_{*}\left(0.175 R_{*}\right)$ & $0.044 R_{*}\left(0.175 R_{*}\right)$ & $0.044 R_{*}\left(0.175 R_{*}\right)$ \\
\hline 3900 & 60 & $0.017 R_{*}\left(0.070 R_{*}\right)$ & $0.026 R_{*}\left(0.105 R_{*}\right)$ & $0.026 R_{*}\left(0.105 R_{*}\right)$ & $0.026 R_{*}\left(0.105 R_{*}\right)$ & $0.026 R_{*}\left(0.105 R_{*}\right)$ & $0.026 R_{*}\left(0.105 R_{*}\right)$ & $0.026 R_{*}\left(0.105 R_{*}\right)$ & $0.026 R_{*}\left(0.105 R_{*}\right)$ & $0.026 R_{*}\left(0.105 R_{*}\right)$ \\
\hline 3900 & 100 & $0.026 R_{*}\left(0.105 R_{*}\right)$ & $0.026 R_{*}\left(0.105 R_{*}\right)$ & $0.035 R_{*}\left(0.140 R_{*}\right)$ & $0.035 R_{*}\left(0.140 R_{*}\right)$ & $0.035 R_{*}\left(0.140 R_{*}\right)$ & $0.035 R_{*}\left(0.140 R_{*}\right)$ & $0.035 R_{*}\left(0.140 R_{*}\right)$ & $0.035 R_{*}\left(0.140 R_{*}\right)$ & $0.035 R_{*}\left(0.140 R_{*}\right)$ \\
\hline 3900 & 150 & $0.035 R_{*}\left(0.140 R_{*}\right)$ & $0.035 R_{*}\left(0.140 R_{*}\right)$ & $0.035 R_{*}\left(0.140 R_{*}\right)$ & $0.035 R_{*}\left(0.140 R_{*}\right)$ & $0.044 R_{*}\left(0.175 R_{*}\right)$ & $0.044 R_{*}\left(0.175 R_{*}\right)$ & $0.044 R_{*}\left(0.175 R_{*}\right)$ & $0.044 R_{*}\left(0.175 R_{*}\right)$ & $0.044 R_{*}\left(0.175 R_{*}\right)$ \\
\hline
\end{tabular}

Notes. For a direct comparison with the results from the M4V dwarf host star simulations, the values of $r_{\text {spot }}$ in brackets are the starspot size if they were on the M4V dwarf. 
A\&A 630, A114 (2019)

Table A.3. continued.

\begin{tabular}{|c|c|c|c|c|c|c|c|c|c|c|}
\hline \multirow{2}{*}{$\begin{array}{l}T_{\text {spot }} \\
(K)\end{array}$} & \multirow{2}{*}{$\begin{array}{l}\text { rms scatter } \\
\text { (ppm) }\end{array}$} & \multicolumn{3}{|c|}{$\begin{array}{c}\lambda_{\mathrm{obs}}=600 \mathrm{~nm} \\
P\end{array}$} & \multicolumn{3}{|c|}{$\begin{array}{c}\lambda_{\mathrm{obs}}=785 \mathrm{~nm} \\
P\end{array}$} & \multicolumn{3}{|c|}{$\begin{array}{c}\lambda_{\mathrm{obs}}=1000 \mathrm{~nm} \\
P\end{array}$} \\
\hline & & $2 \mathrm{~d}$ & $4 \mathrm{~d}$ & $6 \mathrm{~d}$ & $2 \mathrm{~d}$ & $4 \mathrm{~d}$ & $6 \mathrm{~d}$ & $2 \mathrm{~d}$ & $4 \mathrm{~d}$ & $6 \mathrm{~d}$ \\
\hline 3900 & 200 & $0.044 R_{*}\left(0.175 R_{*}\right)$ & $0.044 R_{*}\left(0.175 R_{*}\right)$ & $0.044 R_{*}\left(0.175 R_{*}\right)$ & $0.044 R_{*}\left(0.175 R_{*}\right)$ & $0.044 R_{*}\left(0.175 R_{*}\right)$ & $0.052 R_{*}\left(0.209 R_{*}\right)$ & $0.052 R_{*}\left(0.209 R_{*}\right)$ & $0.052 R_{*}\left(0.209 R_{*}\right)$ & $0.052 R_{*}\left(0.209 R_{*}\right)$ \\
\hline 4000 & 60 & $0.026 R_{*}\left(0.105 R_{*}\right)$ & $0.026 R_{*}\left(0.105 R_{*}\right)$ & $0.035 R_{*}\left(0.140 R_{*}\right)$ & $0.026 R_{*}\left(0.105 R_{*}\right)$ & $0.035 R_{*}\left(0.140 R_{*}\right)$ & $0.035 R_{*}\left(0.140 R_{*}\right)$ & $0.035 R_{*}\left(0.140 R_{*}\right)$ & $0.035 R_{*}\left(0.140 R_{*}\right)$ & $0.035 R_{*}\left(0.140 R_{*}\right)$ \\
\hline 4000 & 100 & $0.035 R_{*}\left(0.140 R_{*}\right)$ & $0.035 R_{*}\left(0.140 R_{*}\right)$ & $0.044 R_{*}\left(0.175 R_{*}\right)$ & $0.044 R_{*}\left(0.175 R_{*}\right)$ & $0.044 R_{*}\left(0.175 R_{*}\right)$ & $0.052 R_{*}\left(0.209 R_{*}\right)$ & $0.044 R_{*}\left(0.175 R_{*}\right)$ & $0.052 R_{*}\left(0.209 R_{*}\right)$ & $0.052 R_{*}\left(0.209 R_{*}\right)$ \\
\hline 4000 & 150 & $0.044 R_{*}\left(0.175 R_{*}\right)$ & $0.052 R_{*}\left(0.209 R_{*}\right)$ & $0.052 R_{*}\left(0.209 R_{*}\right)$ & $0.052 R_{*}\left(0.209 R_{*}\right)$ & $0.052 R_{*}\left(0.209 R_{*}\right)$ & $0.061 R_{*}\left(0.244 R_{*}\right)$ & $0.052 R_{*}\left(0.209 R_{*}\right)$ & $0.061 R_{*}\left(0.244 R_{*}\right)$ & $0.070 R_{*}\left(0.279 R_{*}\right)$ \\
\hline 4000 & 200 & $0.052 R_{*}\left(0.209 R_{*}\right)$ & $0.061 R_{*}\left(0.244 R_{*}\right)$ & $0.061 R_{*}\left(0.244 R_{*}\right)$ & $0.052 R_{*}\left(0.209 R_{*}\right)$ & $0.061 R_{*}\left(0.244 R_{*}\right)$ & $0.070 R_{*}\left(0.279 R_{*}\right)$ & $0.061 R_{*}\left(0.244 R_{*}\right)$ & $0.079 R_{*}\left(0.314 R_{*}\right)$ & $0.087 R_{*}\left(0.349 R_{*}\right)$ \\
\hline
\end{tabular}

$R_{\mathrm{p}}=5.00 R_{\oplus}$

\begin{tabular}{|c|c|c|c|c|c|c|c|c|c|c|}
\hline 3700 & 60 & $0.017 R_{*}\left(0.070 R_{*}\right)$ & $0.017 R_{*}\left(0.070 R_{*}\right)$ & $0.017 R_{*}\left(0.070 R_{*}\right)$ & $0.017 R_{*}\left(0.070 R_{*}\right)$ & $0.017 R_{*}\left(0.070 R_{*}\right)$ & $0.017 R_{*}\left(0.070 R_{*}\right)$ & $0.017 R_{*}\left(0.070 R_{*}\right)$ & $0.017 R_{*}\left(0.070 R_{*}\right)$ & $0.017 R_{*}\left(0.070 R_{*}\right)$ \\
\hline 3700 & 100 & $0.026 R_{*}\left(0.105 R_{*}\right)$ & $0.026 R_{*}\left(0.105 R_{*}\right)$ & $.026 R_{*}\left(0.105 R_{*}\right)$ & $026 R_{*}\left(0.105 R_{*}\right)$ & $0.026 R_{*}\left(0.105 R_{*}\right)$ & $0.026 R_{*}\left(0.105 R_{*}\right)$ & $0.026 R_{*}\left(0.105 R_{*}\right)$ & $0.026 R_{*}\left(0.105 R_{*}\right)$ & $0.026 R_{*}\left(0.105 R_{*}\right)$ \\
\hline 3700 & 150 & $0.026 R_{*}\left(0.105 R_{*}\right)$ & $0.026 R_{*}\left(0.105 R_{*}\right)$ & $0.026 R_{*}\left(0.105 R_{*}\right)$ & $26 R_{*}\left(0.105 R_{*}\right)$ & $0.026 R_{*}\left(0.105 R_{*}\right)$ & $0.026 R_{*}\left(0.105 R_{*}\right)$ & $0.026 R_{*}\left(0.105 R_{*}\right)$ & $0.026 R_{*}\left(0.105 R_{*}\right)$ & $0.035 R_{*}\left(0.140 R_{*}\right)$ \\
\hline 3700 & 200 & $0.026 R_{*}\left(0.105 R_{*}\right)$ & $0.026 R_{*}\left(0.105 R_{*}\right)$ & $0.035 R_{*}\left(0.140 R_{*}\right)$ & $035 R_{*}\left(0.140 R_{*}\right)$ & $0.035 R_{*}\left(0.140 R_{*}\right)$ & $0.035 R_{*}\left(0.140 R_{*}\right)$ & $0.035 R_{*}\left(0.140 R_{*}\right)$ & $0.035 R_{*}\left(0.140 R_{*}\right)$ & $0.044 R_{*}\left(0.175 R_{*}\right)$ \\
\hline 3800 & 60 & $0.017 R_{*}\left(0.070 R_{*}\right)$ & $0.017 R_{*}\left(0.070 R_{*}\right)$ & $0.017 R_{*}\left(0.070 R_{*}\right)$ & $0.017 R_{*}\left(0.070 R_{*}\right)$ & $0.017 R_{*}\left(0.070 R_{*}\right)$ & $0.017 R_{*}\left(0.070 R_{*}\right)$ & $0.017 R_{*}\left(0.070 R_{*}\right)$ & $0.017 R_{*}\left(0.070 R_{*}\right)$ & $0.026 R_{*}\left(0.105 R_{*}\right)$ \\
\hline 3800 & 100 & $026 R_{*}\left(0.105 R_{*}\right)$ & $0.026 R_{*}\left(0.105 R_{*}\right)$ & $0.026 R_{*}\left(0.105 R_{*}\right)$ & $\left..105 R_{*}\right)$ & $0.026 R_{*}\left(0.105 R_{*}\right)$ & $\left..105 R_{*}\right)$ & $0.026 R_{*}\left(0.105 R_{*}\right)$ & $\left.5 R_{*}\right)$ & $\left..105 R_{*}\right)$ \\
\hline 3800 & 150 & $0.026 R_{*}\left(0.105 R_{*}\right)$ & $0.026 R_{*}\left(0.105 R_{*}\right)$ & $0.035 R_{*}\left(0.140 R_{*}\right)$ & $035 R_{*}\left(0.140 R_{*}\right)$ & $35 R_{*}\left(0.140 R_{*}\right)$ & $\left(0.140 R_{*}\right)$ & $R_{*}\left(0.140 R_{*}\right)$ & $\left(0.140 R_{*}\right)$ & $\left..140 R_{*}\right)$ \\
\hline 3800 & 200 & $0.035 R_{*}\left(0.140 R_{*}\right)$ & 0.035 & 0.035 & 0.0 & 0.035 & 0.03. & & 0.04 & $\left.175 R_{*}\right)$ \\
\hline 3900 & 60 & $0.017 R_{*}\left(0.070 R_{*}\right)$ & $0.017 R_{*}\left(0.070 R_{*}\right)$ & $0.026 R_{*}\left(0.105 R_{*}\right)$ & $0.026 R_{*}\left(0.105 R_{*}\right)$ & $0.026 R_{*}\left(0.105 R_{*}\right)$ & $0.026 R_{*}\left(0.105 R_{*}\right)$ & $0.026 R_{*}\left(0.105 R_{*}\right)$ & $0.026 R_{*}\left(0.105 R_{*}\right)$ & $0.026 R_{*}\left(0.105 R_{*}\right)$ \\
\hline 3900 & 100 & $0.026 R_{*}\left(0.105 R_{*}\right)$ & $0.026 R_{*}\left(0.105 R_{*}\right)$ & $0.035 R_{*}\left(0.140 R_{*}\right)$ & $0.026 R_{*}\left(0.105 R_{*}\right)$ & $0.035 R_{*}\left(0.140 R_{*}\right)$ & $0.035 R_{*}\left(0.140 R_{*}\right)$ & $0.035 R_{*}\left(0.140 R_{*}\right)$ & $0.035 R_{*}\left(0.140 R_{*}\right)$ & $0.035 R_{*}\left(0.140 R_{*}\right)$ \\
\hline 3900 & 150 & $0.035 R_{*}\left(0.140 R_{*}\right)$ & $0.035 R_{*}\left(0.140 R_{*}\right)$ & $0.035 R_{*}\left(0.140 R_{*}\right)$ & $0.035 R_{*}\left(0.140 R_{*}\right)$ & $0.035 R_{*}\left(0.140 R_{*}\right)$ & $0.044 R_{*}\left(0.175 R_{*}\right)$ & $0.035 R_{*}\left(0.140 R_{*}\right)$ & $0.044 R_{*}\left(0.175 R_{*}\right)$ & $0.044 R_{*}\left(0.175 R_{*}\right)$ \\
\hline 3900 & 200 & $0.035 R_{*}\left(0.140 R_{*}\right)$ & $0.044 R_{*}\left(0.175 R_{*}\right)$ & $0.044 R_{*}\left(0.175 R_{*}\right)$ & $0.044 R_{*}\left(0.175 R_{*}\right)$ & $0.044 R_{*}\left(0.175 R_{*}\right)$ & $0.044 R_{*}\left(0.175 R_{*}\right)$ & $0.044 R_{*}\left(0.175 R_{*}\right)$ & $0.044 R_{*}\left(0.175 R_{*}\right)$ & $0.052 R_{*}\left(0.209 R_{*}\right)$ \\
\hline 4000 & 60 & $0.026 R_{*}\left(0.105 R_{*}\right)$ & $0.026 R_{*}\left(0.105 R_{*}\right)$ & $0.026 R_{*}\left(0.105 R_{*}\right)$ & $0.035 R_{*}\left(0.140 R_{*}\right)$ & $0.026 R_{*}\left(0.105 R_{*}\right)$ & $0.035 R_{*}\left(0.140 R_{*}\right)$ & $0.035 R_{*}\left(0.140 R_{*}\right)$ & $0.035 R_{*}\left(0.140 R_{*}\right)$ & $0.035 R_{*}\left(0.140 R_{*}\right)$ \\
\hline 4000 & 100 & $0.035 R_{*}\left(0.140 R_{*}\right)$ & $0.035 R_{*}\left(0.140 R_{*}\right)$ & $0.044 R_{*}\left(0.175 R_{*}\right)$ & $0.044 R_{*}\left(0.175 R_{*}\right)$ & $0.044 R_{*}\left(0.175 R_{*}\right)$ & $0.044 R_{*}\left(0.175 R_{*}\right)$ & $0.044 R_{*}\left(0.175 R_{*}\right)$ & $0.044 R_{*}\left(0.175 R_{*}\right)$ & $0.052 R_{*}\left(0.209 R_{*}\right)$ \\
\hline 4000 & 150 & $0.044 R_{*}\left(0.175 R_{*}\right)$ & $0.044 R_{*}\left(0.175 R_{*}\right)$ & $0.052 R_{*}\left(0.209 R_{*}\right)$ & $0.052 R_{*}\left(0.209 R_{*}\right)$ & $0.052 R_{*}\left(0.209 R_{*}\right)$ & $0.052 R_{*}\left(0.209 R_{*}\right)$ & $0.061 R_{*}\left(0.244 R_{*}\right)$ & $0.061 R_{*}\left(0.244 R_{*}\right)$ & $0.061 R_{*}\left(0.244 R_{*}\right)$ \\
\hline 4000 & 200 & $0.052 R_{*}\left(0.209 R_{*}\right)$ & $0.052 R_{*}\left(0.209 R_{*}\right)$ & $0.061 R_{*}\left(0.244 R_{*}\right)$ & $0.061 R_{*}\left(0.244 R_{*}\right)$ & $0.061 R_{*}\left(0.244 R_{*}\right)$ & $0.070 R_{*}\left(0.279 R_{*}\right)$ & $0.070 R_{*}\left(0.279 R_{*}\right)$ & $0.070 R_{*}\left(0.279 R_{*}\right)$ & $0.079 R_{*}\left(0.314 R_{*}\right)$ \\
\hline
\end{tabular}

$R_{\mathrm{p}}=5.50 R_{\oplus}$

\begin{tabular}{|c|c|c|c|c|c|c|c|c|c|c|}
\hline 3700 & 60 & $0.017 R_{*}\left(0.070 R_{*}\right)$ & $0.017 R_{*}\left(0.070 R_{*}\right)$ & $0.017 R_{*}\left(0.070 R_{*}\right)$ & $0.017 R_{*}\left(0.070 R_{*}\right)$ & $0.017 R_{*}\left(0.070 R_{*}\right)$ & $0.017 R_{*}\left(0.070 R_{*}\right)$ & $0.017 R_{*}\left(0.070 R_{*}\right)$ & $0.017 R_{*}\left(0.070 R_{*}\right)$ & $0.017 R_{*}($ \\
\hline 3700 & 100 & $0.026 R_{*}\left(0.105 R_{*}\right)$ & $0.026 R_{*}\left(0.105 R_{*}\right)$ & $0.026 R_{*}\left(0.105 R_{*}\right)$ & $0.026 R_{*}\left(0.105 R_{*}\right)$ & $0.026 R_{*}\left(0.105 R_{*}\right)$ & $0.026 R_{*}\left(0.105 R_{*}\right)$ & $0.026 R_{*}\left(0.105 R_{*}\right)$ & $0.026 R_{*}\left(0.105 R_{*}\right)$ & $0.026 R_{*}\left(0.105 R_{*}\right)$ \\
\hline 3700 & 150 & $0.026 R_{*}\left(0.105 R_{*}\right)$ & $0.026 R_{*}\left(0.105 R_{*}\right)$ & $0.026 R_{*}\left(0.105 R_{*}\right)$ & $026 R_{*}\left(0.105 R_{*}\right)$ & $0.026 R_{*}\left(0.105 R_{*}\right)$ & $0.026 R_{*}\left(0.105 R_{*}\right)$ & $0.026 R_{*}\left(0.105 R_{*}\right)$ & $0.035 R_{*}\left(0.140 R_{*}\right)$ & $0.035 R_{*}\left(0.140 R_{*}\right)$ \\
\hline 3700 & 200 & $026 R_{*}\left(0.105 R_{*}\right)$ & $0.026 R_{*}\left(0.105 R_{*}\right)$ & $0.035 R_{*}\left(0.140 R_{*}\right)$ & $035 R_{*}\left(0.140 R_{*}\right)$ & $0.035 R_{*}\left(0.140 R_{*}\right)$ & $0.035 R_{*}\left(0.140 R_{*}\right)$ & $0.035 R_{*}\left(0.140 R_{*}\right)$ & $0.035 R_{*}\left(0.140 R_{*}\right)$ & $0.035 R_{*}\left(0.140 R_{*}\right)$ \\
\hline 3800 & 60 & $0.017 R_{*}\left(0.070 R_{*}\right)$ & $0.017 R_{*}\left(0.070 R_{*}\right)$ & $0.017 R_{*}\left(0.070 R_{*}\right)$ & $0.026 R_{*}\left(0.105 R_{*}\right)$ & $0.026 R_{*}\left(0.105 R_{*}\right)$ & $0.026 R_{*}\left(0.105 R_{*}\right)$ & $0.026 R_{*}\left(0.105 R_{*}\right)$ & $0.026 R_{*}\left(0.105 R_{*}\right)$ & $0.026 R_{*}\left(0.105 R_{*}\right)$ \\
\hline 3800 & 100 & $0.026 R_{*}\left(0.105 R_{*}\right)$ & $0.026 R_{*}\left(0.105 R_{*}\right)$ & $0.026 R_{*}\left(0.105 R_{*}\right)$ & $0.026 R_{*}\left(0.105 R_{*}\right)$ & $0.026 R_{*}\left(0.105 R_{*}\right)$ & $0.026 R_{*}\left(0.105 R_{*}\right)$ & $0.026 R_{*}\left(0.105 R_{*}\right)$ & $0.026 R_{*}\left(0.105 R_{*}\right)$ & $0.026 R_{*}\left(0.105 R_{*}\right)$ \\
\hline 3800 & 150 & $0.026 R_{*}\left(0.105 R_{*}\right)$ & $0.026 R_{*}\left(0.105 R_{*}\right)$ & $0.035 R_{*}\left(0.140 R_{*}\right)$ & $0.035 R_{*}\left(0.140 R_{*}\right)$ & $0.035 R_{*}\left(0.140 R_{*}\right)$ & $0.035 R_{*}\left(0.140 R_{*}\right)$ & $0.035 R_{*}\left(0.140 R_{*}\right)$ & $0.035 R_{*}\left(0.140 R_{*}\right)$ & $0.035 R_{*}\left(0.140 R_{*}\right)$ \\
\hline 3800 & 200 & $0.035 R_{*}\left(0.140 R_{*}\right)$ & $0.035 R_{*}\left(0.140 R_{*}\right)$ & $0.035 R_{*}\left(0.140 R_{*}\right)$ & $0.035 R_{*}\left(0.140 R_{*}\right)$ & $0.035 R_{*}\left(0.140 R_{*}\right)$ & $0.035 R_{*}\left(0.140 R_{*}\right)$ & $0.044 R_{*}\left(0.175 R_{*}\right)$ & $0.035 R_{*}\left(0.140 R_{*}\right)$ & $0.044 R_{*}\left(0.175 R_{*}\right)$ \\
\hline 3900 & 60 & $0.026 R_{*}\left(0.105 R_{*}\right)$ & $0.026 R_{*}\left(0.105 R_{*}\right)$ & $0.026 R_{*}\left(0.105 R_{*}\right)$ & $0.026 R_{*}\left(0.105 R_{*}\right)$ & $0.026 R_{*}\left(0.105 R_{*}\right)$ & $0.026 R_{*}\left(0.105 R_{*}\right)$ & $0.026 R_{*}\left(0.105 R_{*}\right)$ & $0.026 R_{*}\left(0.105 R_{*}\right)$ & $0.026 R_{*}\left(0.105 R_{*}\right)$ \\
\hline 3900 & 100 & $0.026 R_{*}\left(0.105 R_{*}\right)$ & $0.026 R_{*}\left(0.105 R_{*}\right)$ & $0.026 R_{*}\left(0.105 R_{*}\right)$ & $0.035 R_{*}\left(0.140 R_{*}\right)$ & $0.026 R_{*}\left(0.105 R_{*}\right)$ & $0.035 R_{*}\left(0.140 R_{*}\right)$ & $0.035 R_{*}\left(0.140 R_{*}\right)$ & $0.035 R_{*}\left(0.140 R_{*}\right)$ & $0.035 R_{*}\left(0.140 R_{*}\right)$ \\
\hline 3900 & 150 & $0.035 R_{*}\left(0.140 R_{*}\right)$ & $0.035 R_{*}\left(0.140 R_{*}\right)$ & $0.035 R_{*}\left(0.140 R_{*}\right)$ & $0.035 R_{*}\left(0.140 R_{*}\right)$ & $0.035 R_{*}\left(0.140 R_{*}\right)$ & $0.035 R_{*}\left(0.140 R_{*}\right)$ & $0.044 R_{*}\left(0.175 R_{*}\right)$ & $0.044 R_{*}\left(0.175 R_{*}\right)$ & $0.044 R_{*}\left(0.175 R_{*}\right)$ \\
\hline 3900 & 200 & $0.035 R_{*}\left(0.140 R_{*}\right)$ & $0.035 R_{*}\left(0.140 R_{*}\right)$ & $0.044 R_{*}\left(0.175 R_{*}\right)$ & $0.044 R_{*}\left(0.175 R_{*}\right)$ & $0.044 R_{*}\left(0.175 R_{*}\right)$ & $0.044 R_{*}\left(0.175 R_{*}\right)$ & $0.044 R_{*}\left(0.175 R_{*}\right)$ & $0.052 R_{*}\left(0.209 R_{*}\right)$ & $0.052 R_{*}\left(0.209 R_{*}\right)$ \\
\hline 4000 & 60 & $0.026 R_{*}\left(0.105 R_{*}\right)$ & $0.026 R_{*}\left(0.105 R_{*}\right)$ & $0.035 R_{*}\left(0.140 R_{*}\right)$ & $0.035 R_{*}\left(0.140 R_{*}\right)$ & $0.035 R_{*}\left(0.140 R_{*}\right)$ & $0.035 R_{*}\left(0.140 R_{*}\right)$ & $0.035 R_{*}\left(0.140 R_{*}\right)$ & $0.035 R_{*}\left(0.140 R_{*}\right)$ & $0.035 R_{*}\left(0.140 R_{*}\right)$ \\
\hline 4000 & 100 & $0.035 R_{*}\left(0.140 R_{*}\right)$ & $0.035 R_{*}\left(0.140 R_{*}\right)$ & $0.044 R_{*}\left(0.175 R_{*}\right)$ & $0.044 R_{*}\left(0.175 R_{*}\right)$ & $0.044 R_{*}\left(0.175 R_{*}\right)$ & $0.044 R_{*}\left(0.175 R_{*}\right)$ & $0.044 R_{*}\left(0.175 R_{*}\right)$ & $0.044 R_{*}\left(0.175 R_{*}\right)$ & $0.052 R_{*}\left(0.209 R_{*}\right)$ \\
\hline 4000 & 150 & $0.044 R_{*}\left(0.175 R_{*}\right)$ & $0.044 R_{*}\left(0.175 R_{*}\right)$ & $0.044 R_{*}\left(0.175 R_{*}\right)$ & $0.052 R_{*}\left(0.209 R_{*}\right)$ & $0.052 R_{*}\left(0.209 R_{*}\right)$ & $0.052 R_{*}\left(0.209 R_{*}\right)$ & $0.052 R_{*}\left(0.209 R_{*}\right)$ & $0.061 R_{*}\left(0.244 R_{*}\right)$ & $0.061 R_{*}(0.244 K$ \\
\hline 4000 & 200 & $0.052 R_{*}\left(0.209 R_{*}\right)$ & $0.052 R_{*}\left(0.209 R_{*}\right)$ & $0.052 R_{*}\left(0.209 R_{*}\right)$ & $0.061 R_{*}\left(0.244 R_{*}\right)$ & $0.061 R_{*}\left(0.244 R_{*}\right)$ & $0.061 R_{*}\left(0.244 R_{*}\right)$ & $0.061 R_{*}\left(0.244 R_{*}\right)$ & $0.070 R_{*}\left(0.279 R_{*}\right)$ & $0.070 R_{*}\left(0.279 R_{*}\right)$ \\
\hline
\end{tabular}

\begin{tabular}{rrr}
\hline $\mathrm{p}=6.00 R_{\oplus}$ \\
\hline
\end{tabular}

\begin{tabular}{|c|c|c|c|c|c|c|c|c|c|c|}
\hline 3700 & 60 & $0.017 R_{*}\left(0.070 R_{*}\right)$ & $0.017 R_{*}\left(0.070 R_{*}\right)$ & $0.017 R_{*}\left(0.070 R_{*}\right)$ & $0.017 R_{*}\left(0.070 R_{*}\right)$ & $0.017 R_{*}\left(0.070 R_{*}\right)$ & $0.017 R_{*}\left(0.070 R_{*}\right)$ & $0.026 R_{*}\left(0.105 R_{*}\right)$ & $0.026 R_{*}\left(0.105 R_{*}\right)$ & $0.026 R_{*}\left(0.105 R_{*}\right)$ \\
\hline 3700 & 100 & $0.017 R_{*}\left(0.070 R_{*}\right)$ & $026 R_{*}\left(0.105 R_{*}\right)$ & $.026 R_{*}\left(0.105 R_{*}\right)$ & $0.026 R_{*}\left(0.105 R_{*}\right)$ & $0.026 R_{*}\left(0.105 R_{*}\right)$ & $0.026 R_{*}\left(0.105 R_{*}\right)$ & $0.026 R_{*}\left(0.105 R_{*}\right)$ & $0.026 R_{*}\left(0.105 R_{*}\right)$ & $0.026 R_{*}\left(0.105 R_{*}\right)$ \\
\hline 3700 & 150 & $0.026 R_{*}\left(0.105 R_{*}\right)$ & $0.026 R_{*}\left(0.105 R_{*}\right)$ & $0.026 R_{*}\left(0.105 R_{*}\right)$ & $0.026 R_{*}\left(0.105 R_{*}\right)$ & $0.026 R_{*}\left(0.105 R_{*}\right)$ & $0.026 R_{*}\left(0.105 R_{*}\right)$ & $0.026 R_{*}\left(0.105 R_{*}\right)$ & $0.035 R_{*}\left(0.140 R_{*}\right)$ & $0.026 R_{*}\left(0.105 R_{*}\right)$ \\
\hline 3700 & 200 & $0.026 R_{*}\left(0.105 R_{*}\right)$ & $0.026 R_{*}\left(0.105 R_{*}\right)$ & $0.026 R_{*}\left(0.105 R_{*}\right)$ & $0.035 R_{*}\left(0.140 R_{*}\right)$ & $0.035 R_{*}\left(0.140 R_{*}\right)$ & $0.035 R_{*}\left(0.140 R_{*}\right)$ & $0.035 R_{*}\left(0.140 R_{*}\right)$ & $0.035 R_{*}\left(0.140 R_{*}\right)$ & $0.035 R_{*}\left(0.140 R_{*}\right)$ \\
\hline 3800 & 60 & $0.017 R_{*}\left(0.070 R_{*}\right)$ & $0.017 R_{*}\left(0.070 R_{*}\right)$ & $0.017 R_{*}\left(0.070 R_{*}\right)$ & $0.026 R_{*}\left(0.105 R_{*}\right)$ & $0.026 R_{*}\left(0.105 R_{*}\right)$ & $0.026 R_{*}\left(0.105 R_{*}\right)$ & $0.026 R_{*}\left(0.105 R_{*}\right)$ & $0.026 R_{*}\left(0.105 R_{*}\right)$ & $0.026 R_{*}\left(0.105 R_{*}\right)$ \\
\hline 3800 & 100 & $0.026 R_{*}\left(0.105 R_{*}\right)$ & $0.026 R_{*}\left(0.105 R_{*}\right)$ & $026 R_{*}\left(0.105 R_{*}\right)$ & $0.026 R_{*}\left(0.105 R_{*}\right)$ & $0.026 R_{*}\left(0.105 R_{*}\right)$ & $0.026 R_{*}\left(0.105 R_{*}\right)$ & $0.026 R_{*}\left(0.105 R_{*}\right)$ & $0.026 R_{*}\left(0.105 R_{*}\right)$ & $0.026 R_{*}\left(0.105 R_{*}\right)$ \\
\hline 3800 & 150 & $0.026 R_{*}\left(0.105 R_{*}\right)$ & $0.026 R_{*}\left(0.105 R_{*}\right)$ & $0.026 R_{*}\left(0.105 R_{*}\right)$ & $0.035 R_{*}\left(0.140 R_{*}\right)$ & $0.035 R_{*}\left(0.140 R_{*}\right)$ & $0.035 R_{*}\left(0.140 R_{*}\right)$ & $0.035 R_{*}\left(0.140 R_{*}\right)$ & $0.035 R_{*}\left(0.140 R_{*}\right)$ & $0.035 R_{*}\left(0.140 R_{*}\right)$ \\
\hline 3800 & 200 & $0.035 R_{*}\left(0.140 R_{*}\right)$ & $0.035 R_{*}\left(0.140 R_{*}\right)$ & $0.035 R_{*}\left(0.140 R_{*}\right)$ & $0.035 R_{*}\left(0.140 R_{*}\right)$ & $0.035 R_{*}\left(0.140 R_{*}\right)$ & $0.035 R_{*}\left(0.140 R_{*}\right)$ & $0.035 R_{*}\left(0.140 R_{*}\right)$ & $0.035 R_{*}\left(0.140 R_{*}\right)$ & $0.044 R_{*}\left(0.175 R_{*}\right)$ \\
\hline 3900 & 60 & $0.026 R_{*}\left(0.105 R_{*}\right)$ & $0.026 R_{*}\left(0.105 R_{*}\right)$ & $0.026 R_{*}\left(0.105 R_{*}\right)$ & $0.026 R_{*}\left(0.105 R_{*}\right)$ & $0.026 R_{*}\left(0.105 R_{*}\right)$ & $0.026 R_{*}\left(0.105 R_{*}\right)$ & $0.026 R_{*}\left(0.105 R_{*}\right)$ & $0.026 R_{*}\left(0.105 R_{*}\right)$ & $0.026 R_{*}\left(0.105 R_{*}\right)$ \\
\hline 3900 & 100 & $0.026 R_{*}\left(0.105 R_{*}\right)$ & $0.026 R_{*}\left(0.105 R_{*}\right)$ & $0.026 R_{*}\left(0.105 R_{*}\right)$ & $0.035 R_{*}\left(0.140 R_{*}\right)$ & $0.026 R_{*}\left(0.105 R_{*}\right)$ & $0.035 R_{*}\left(0.140 R_{*}\right)$ & $0.035 R_{*}\left(0.140 R_{*}\right)$ & $0.035 R_{*}\left(0.140 R_{*}\right)$ & $0.035 R_{*}\left(0.140 R_{*}\right)$ \\
\hline 3900 & 150 & $0.035 R_{*}\left(0.140 R_{*}\right)$ & $0.035 R_{*}\left(0.140 R_{*}\right)$ & $0.035 R_{*}\left(0.140 R_{*}\right)$ & $0.035 R_{*}\left(0.140 R_{*}\right)$ & $0.035 R_{*}\left(0.140 R_{*}\right)$ & $0.035 R_{*}\left(0.140 R_{*}\right)$ & $0.044 R_{*}\left(0.175 R_{*}\right)$ & $0.044 R_{*}\left(0.175 R_{*}\right)$ & $0.044 R_{*}\left(0.175 R_{*}\right)$ \\
\hline 3900 & 200 & $0.035 R_{*}\left(0.140 R_{*}\right)$ & $0.035 R_{*}\left(0.140 R_{*}\right)$ & $0.035 R_{*}\left(0.140 R_{*}\right)$ & $0.044 R_{*}\left(0.175 R_{*}\right)$ & $0.044 R_{*}\left(0.175 R_{*}\right)$ & $0.044 R_{*}\left(0.175 R_{*}\right)$ & $0.044 R_{*}\left(0.175 R_{*}\right)$ & $0.044 R_{*}\left(0.175 R_{*}\right)$ & $0.044 R_{*}\left(0.175 R_{*}\right)$ \\
\hline 4000 & 60 & $0.035 R_{*}\left(0.140 R_{*}\right)$ & $0.035 R_{*}\left(0.140 R_{*}\right)$ & $0.035 R_{*}\left(0.140 R_{*}\right)$ & $0.035 R_{*}\left(0.140 R_{*}\right)$ & $0.035 R_{*}\left(0.140 R_{*}\right)$ & $0.035 R_{*}\left(0.140 R_{*}\right)$ & $0.035 R_{*}\left(0.140 R_{*}\right)$ & $0.035 R_{*}\left(0.140 R_{*}\right)$ & $0.044 R_{*}\left(0.175 R_{*}\right)$ \\
\hline 4000 & 100 & $0.035 R_{*}\left(0.140 R_{*}\right)$ & $0.035 R_{*}\left(0.140 R_{*}\right)$ & $0.035 R_{*}\left(0.140 R_{*}\right)$ & $0.044 R_{*}\left(0.175 R_{*}\right)$ & $0.044 R_{*}\left(0.175 R_{*}\right)$ & $0.044 R_{*}\left(0.175 R_{*}\right)$ & $0.044 R_{*}\left(0.175 R_{*}\right)$ & $0.044 R_{*}\left(0.175 R_{*}\right)$ & $0.044 R_{*}\left(0.175 R_{*}\right)$ \\
\hline 4000 & 150 & $0.044 R_{*}\left(0.175 R_{*}\right)$ & $0.044 R_{*}\left(0.175 R_{*}\right)$ & $0.044 R_{*}\left(0.175 R_{*}\right)$ & $0.052 R_{*}\left(0.209 R_{*}\right)$ & $0.052 R_{*}\left(0.209 R_{*}\right)$ & $0.052 R_{*}\left(0.209 R_{*}\right)$ & $0.052 R_{*}\left(0.209 R_{*}\right)$ & $0.052 R_{*}\left(0.209 R_{*}\right)$ & $0.061 R_{*}\left(0.244 R_{*}\right)$ \\
\hline 4000 & 200 & $0.052 R_{*}\left(0.209 R_{*}\right)$ & $0.052 R_{*}\left(0.209 R_{*}\right)$ & $0.052 R_{*}\left(0.209 R_{*}\right)$ & $0.061 R_{*}\left(0.244 R_{*}\right)$ & $0.061 R_{*}\left(0.244 R_{*}\right)$ & $0.061 R_{*}\left(0.244 R_{*}\right)$ & $0.061 R_{*}\left(0.244 R_{*}\right)$ & $0.070 R_{*}\left(0.279 R_{*}\right)$ & $0.070 R_{*}\left(0.279 R_{*}\right)$ \\
\hline \multicolumn{11}{|c|}{$R_{\mathrm{p}}=6.50 R_{\oplus}$} \\
\hline 3700 & 60 & $0.017 R_{*}\left(0.070 R_{*}\right)$ & $0.017 R_{*}\left(0.070 R_{*}\right)$ & $0.017 R_{*}\left(0.070 R_{*}\right)$ & $0.026 R_{*}\left(0.105 R_{*}\right)$ & $0.026 R_{*}($ & $0.026 R_{*}\left(0.105 R_{*}\right)$ & $0.026 R_{*}($ & $0.026 R_{*}\left(0.105 R_{*}\right)$ & $0.026 R_{*}\left(0.105 R_{*}\right)$ \\
\hline 3700 & 100 & $0.026 R_{*}\left(0.105 R_{*}\right)$ & $0.026 R_{*}\left(0.105 R_{*}\right)$ & $0.026 R_{*}\left(0.105 R_{*}\right)$ & $0.026 R_{*}\left(0.105 R_{*}\right)$ & $0.026 R_{*}\left(0.105 R_{*}\right)$ & $0.026 R_{*}\left(0.105 R_{*}\right)$ & $0.026 R_{*}\left(0.105 R_{*}\right)$ & $0.026 R_{*}\left(0.105 R_{*}\right)$ & $0.026 R_{*}\left(0.105 R_{*}\right)$ \\
\hline 3700 & 150 & $0.026 R_{*}\left(0.105 R_{*}\right)$ & $0.026 R_{*}\left(0.105 R_{*}\right)$ & $0.026 R_{*}\left(0.105 R_{*}\right)$ & $0.026 R_{*}\left(0.105 R_{*}\right)$ & $0.026 R_{*}\left(0.105 R_{*}\right)$ & $0.026 R_{*}\left(0.105 R_{*}\right)$ & $0.035 R_{*}\left(0.140 R_{*}\right)$ & $0.035 R_{*}\left(0.140 R_{*}\right)$ & $0.035 R_{*}\left(0.140 R_{*}\right)$ \\
\hline 3700 & 200 & $0.026 R_{*}\left(0.105 R_{*}\right)$ & $0.026 R_{*}\left(0.105 R_{*}\right)$ & $0.026 R_{*}\left(0.105 R_{*}\right)$ & $0.035 R_{*}\left(0.140 R_{*}\right)$ & $0.035 R_{*}\left(0.140 R_{*}\right)$ & $0.035 R_{*}\left(0.140 R_{*}\right)$ & $0.035 R_{*}\left(0.140 R_{*}\right)$ & $0.035 R_{*}\left(0.140 R_{*}\right)$ & $0.035 R_{*}\left(0.140 R_{*}\right)$ \\
\hline 3800 & 60 & $0.026 R_{*}\left(0.105 R_{*}\right)$ & $0.026 R_{*}\left(0.105 R_{*}\right)$ & $0.026 R_{*}\left(0.105 R_{*}\right)$ & $0.026 R_{*}\left(0.105 R_{*}\right)$ & $0.026 R_{*}\left(0.105 R_{*}\right)$ & $0.026 R_{*}\left(0.105 R_{*}\right)$ & $0.026 R_{*}\left(0.105 R_{*}\right)$ & $0.026 R_{*}\left(0.105 R_{*}\right)$ & $0.026 R_{*}\left(0.105 R_{*}\right)$ \\
\hline 3800 & 100 & $0.026 R_{*}\left(0.105 R_{*}\right)$ & $0.026 R_{*}\left(0.105 R_{*}\right)$ & $0.026 R_{*}\left(0.105 R_{*}\right)$ & $0.026 R_{*}\left(0.105 R_{*}\right)$ & $0.026 R_{*}\left(0.105 R_{*}\right)$ & $0.026 R_{*}\left(0.105 R_{*}\right)$ & $0.026 R_{*}\left(0.105 R_{*}\right)$ & $0.026 R_{*}\left(0.105 R_{*}\right)$ & $0.026 R_{*}\left(0.105 R_{*}\right)$ \\
\hline 3800 & 150 & $0.026 R_{*}\left(0.105 R_{*}\right)$ & $0.026 R_{*}\left(0.105 R_{*}\right)$ & $0.026 R_{*}\left(0.105 R_{*}\right)$ & $0.035 R_{*}\left(0.140 R_{*}\right)$ & $0.035 R_{*}\left(0.140 R_{*}\right)$ & $0.035 R_{*}\left(0.140 R_{*}\right)$ & $0.035 R_{*}\left(0.140 R_{*}\right)$ & $0.035 R_{*}\left(0.140 R_{*}\right)$ & $0.035 R_{*}\left(0.140 R_{*}\right)$ \\
\hline 3800 & 200 & $0.035 R_{*}\left(0.140 R_{*}\right)$ & $0.035 R_{*}\left(0.140 R_{*}\right)$ & $0.035 R_{*}\left(0.140 R_{*}\right)$ & $0.035 R_{*}\left(0.140 R_{*}\right)$ & $0.035 R_{*}\left(0.140 R_{*}\right)$ & $0.035 R_{*}\left(0.140 R_{*}\right)$ & $0.035 R_{*}\left(0.140 R_{*}\right)$ & $0.044 R_{*}\left(0.175 R_{*}\right)$ & $0.044 R_{*}\left(0.175 R_{*}\right)$ \\
\hline 3900 & 60 & $0.026 R_{*}\left(0.105 R_{*}\right)$ & $0.026 R_{*}\left(0.105 R_{*}\right)$ & $0.026 R_{*}\left(0.105 R_{*}\right)$ & $0.026 R_{*}\left(0.105 R_{*}\right)$ & $0.026 R_{*}\left(0.105 R_{*}\right)$ & $0.026 R_{*}\left(0.105 R_{*}\right)$ & $0.035 R_{*}\left(0.140 R_{*}\right)$ & $0.035 R_{*}\left(0.140 R_{*}\right)$ & $0.035 R_{*}\left(0.140 R_{*}\right)$ \\
\hline
\end{tabular}


J. Tregloan-Reed and E. Unda-Sanzana: Simulations of starspot anomalies within TESS

Table A.3. continued.

\begin{tabular}{|c|c|c|c|c|c|c|c|c|c|c|}
\hline \multirow{2}{*}{$\begin{array}{l}T_{\text {spot }} \\
(K)\end{array}$} & \multirow{2}{*}{$\begin{array}{l}\text { rms scatter } \\
\text { (ppm) }\end{array}$} & \multicolumn{3}{|c|}{$\begin{array}{c}\lambda_{\mathrm{obs}}=600 \mathrm{~nm} \\
P\end{array}$} & \multicolumn{3}{|c|}{$\begin{array}{c}\lambda_{\mathrm{obs}}=785 \mathrm{~nm} \\
P\end{array}$} & \multicolumn{3}{|c|}{$\begin{array}{c}\lambda_{\mathrm{obs}}=1000 \mathrm{~nm} \\
P\end{array}$} \\
\hline & & $2 \mathrm{~d}$ & $4 \mathrm{~d}$ & $6 \mathrm{~d}$ & $2 \mathrm{~d}$ & $4 \mathrm{~d}$ & $6 \mathrm{~d}$ & $2 \mathrm{~d}$ & $4 \mathrm{~d}$ & $6 \mathrm{~d}$ \\
\hline 3900 & 100 & $0.026 R_{*}\left(0.105 R_{*}\right)$ & $0.026 R_{*}\left(0.105 R_{*}\right)$ & $0.026 R_{*}\left(0.105 R_{*}\right)$ & $0.035 R_{*}\left(0.140 R_{*}\right)$ & $0.035 R_{*}\left(0.140 R_{*}\right)$ & $0.035 R_{*}\left(0.140 R_{*}\right)$ & $0.035 R_{*}\left(0.140 R_{*}\right)$ & $0.035 R_{*}\left(0.140 R_{*}\right)$ & $0.035 R_{*}\left(0.140 R_{*}\right)$ \\
\hline 3900 & 150 & $0.035 R_{*}\left(0.140 R_{*}\right)$ & $0.035 R_{*}\left(0.140 R_{*}\right)$ & $0.035 R_{*}\left(0.140 R_{*}\right)$ & $0.035 R_{*}\left(0.140 R_{*}\right)$ & $0.035 R_{*}\left(0.140 R_{*}\right)$ & $0.035 R_{*}\left(0.140 R_{*}\right)$ & $0.035 R_{*}\left(0.140 R_{*}\right)$ & $0.044 R_{*}\left(0.175 R_{*}\right)$ & $0.044 R_{*}\left(0.175 R_{*}\right)$ \\
\hline 3900 & 200 & $0.035 R_{*}\left(0.140 R_{*}\right)$ & $0.035 R_{*}\left(0.140 R_{*}\right)$ & $0.035 R_{*}\left(0.140 R_{*}\right)$ & $0.044 R_{*}\left(0.175 R_{*}\right)$ & $0.044 R_{*}\left(0.175 R_{*}\right)$ & $0.044 R_{*}\left(0.175 R_{*}\right)$ & $0.044 R_{*}\left(0.175 R_{*}\right)$ & $0.044 R_{*}\left(0.175 R_{*}\right)$ & $0.044 R_{*}\left(0.175 R_{*}\right)$ \\
\hline 4000 & 60 & $0.035 R_{*}\left(0.140 R_{*}\right)$ & $0.035 R_{*}\left(0.140 R_{*}\right)$ & $0.035 R_{*}\left(0.140 R_{*}\right)$ & $0.035 R_{*}\left(0.140 R_{*}\right)$ & $0.035 R_{*}\left(0.140 R_{*}\right)$ & $0.035 R_{*}\left(0.140 R_{*}\right)$ & $0.044 R_{*}\left(0.175 R_{*}\right)$ & $0.044 R_{*}\left(0.175 R_{*}\right)$ & $0.044 R_{*}\left(0.175 R_{*}\right)$ \\
\hline 4000 & 100 & $0.035 R_{*}\left(0.140 R_{*}\right)$ & $0.035 R_{*}\left(0.140 R_{*}\right)$ & $0.035 R_{*}\left(0.140 R_{*}\right)$ & $0.044 R_{*}\left(0.175 R_{*}\right)$ & $0.044 R_{*}\left(0.175 R_{*}\right)$ & $0.044 R_{*}\left(0.175 R_{*}\right)$ & $0.044 R_{*}\left(0.175 R_{*}\right)$ & $0.044 R_{*}\left(0.175 R_{*}\right)$ & $0.044 R_{*}\left(0.175 R_{*}\right)$ \\
\hline 4000 & 150 & $0.044 R_{*}\left(0.175 R_{*}\right)$ & $0.044 R_{*}\left(0.175 R_{*}\right)$ & $0.044 R_{*}\left(0.175 R_{*}\right)$ & $0.052 R_{*}\left(0.209 R_{*}\right)$ & $0.052 R_{*}\left(0.209 R_{*}\right)$ & $0.052 R_{*}\left(0.209 R_{*}\right)$ & $0.052 R_{*}\left(0.209 R_{*}\right)$ & $0.052 R_{*}\left(0.209 R_{*}\right)$ & $0.061 R_{*}\left(0.244 R_{*}\right)$ \\
\hline 4000 & 200 & $0.052 R_{*}\left(0.209 R_{*}\right)$ & $0.052 R_{*}\left(0.209 R_{*}\right)$ & $0.052 R_{*}\left(0.209 R_{*}\right)$ & $0.052 R_{*}\left(0.209 R_{*}\right)$ & $0.052 R_{*}\left(0.209 R_{*}\right)$ & $0.061 R_{*}\left(0.244 R_{*}\right)$ & $0.061 R_{*}\left(0.244 R_{*}\right)$ & $0.061 R_{*}\left(0.244 R_{*}\right)$ & $0.070 R_{*}\left(0.279 R_{*}\right)$ \\
\hline \multicolumn{11}{|c|}{$R_{\mathrm{p}}=7.00 R_{\oplus}$} \\
\hline 3700 & 60 & $0.017 R_{*}\left(0.070 R_{*}\right)$ & $0.017 R_{*}\left(0.070 R_{*}\right)$ & $0.017 R_{*}\left(0.070 R_{*}\right)$ & $0.026 R_{*}\left(0.105 R_{*}\right)$ & $0.026 R_{*}\left(0.105 R_{*}\right)$ & $0.026 R_{*}\left(0.105 R_{*}\right)$ & $0.026 R_{*}\left(0.105 R_{*}\right)$ & $0.026 R_{*}\left(0.105 R_{*}\right)$ & $0.026 R_{*}\left(0.105 R_{*}\right)$ \\
\hline 3700 & 100 & $0.017 R_{*}\left(0.070 R_{*}\right)$ & $0.026 R_{*}\left(0.105 R_{*}\right)$ & $0.017 R_{*}\left(0.070 R_{*}\right)$ & $0.026 R_{*}\left(0.105 R_{*}\right)$ & $0.026 R_{*}\left(0.105 R_{*}\right)$ & $0.026 R_{*}\left(0.105 R_{*}\right)$ & $0.026 R_{*}\left(0.105 R_{*}\right)$ & $0.026 R_{*}\left(0.105 R_{*}\right)$ & $0.026 R_{*}\left(0.105 R_{*}\right)$ \\
\hline 3700 & 150 & $0.026 R_{*}\left(0.105 R_{*}\right)$ & $0.026 R_{*}\left(0.105 R_{*}\right)$ & $0.026 R_{*}\left(0.105 R_{*}\right)$ & $0.026 R_{*}\left(0.105 R_{*}\right)$ & $0.026 R_{*}\left(0.105 R_{*}\right)$ & $0.026 R_{*}\left(0.105 R_{*}\right)$ & $0.026 R_{*}\left(0.105 R_{*}\right)$ & $0.035 R_{*}\left(0.140 R_{*}\right)$ & $0.035 R_{*}\left(0.140 R_{*}\right)$ \\
\hline 3700 & 200 & $0.026 R_{*}\left(0.105 R_{*}\right)$ & $0.026 R_{*}\left(0.105 R_{*}\right)$ & $0.026 R_{*}\left(0.105 R_{*}\right)$ & $0.035 R_{*}\left(0.140 R_{*}\right)$ & $0.035 R_{*}\left(0.140 R_{*}\right)$ & $0.035 R_{*}\left(0.140 R_{*}\right)$ & $0.035 R_{*}\left(0.140 R_{*}\right)$ & $0.035 R_{*}\left(0.140 R_{*}\right)$ & $0.035 R_{*}\left(0.140 R_{*}\right)$ \\
\hline 3800 & 60 & $0.026 R_{*}\left(0.105 R_{*}\right)$ & $0.026 R_{*}\left(0.105 R_{*}\right)$ & $0.026 R_{*}\left(0.105 R_{*}\right)$ & $0.026 R_{*}\left(0.105 R_{*}\right)$ & $0.026 R_{*}\left(0.105 R_{*}\right)$ & $0.026 R_{*}\left(0.105 R_{*}\right)$ & $0.026 R_{*}\left(0.105 R_{*}\right)$ & $0.026 R_{*}\left(0.105 R_{*}\right)$ & $0.026 R_{*}\left(0.105 R_{*}\right)$ \\
\hline 3800 & 100 & $0.026 R_{*}\left(0.105 R_{*}\right)$ & $0.026 R_{*}\left(0.105 R_{*}\right)$ & $0.026 R_{*}\left(0.105 R_{*}\right)$ & $0.026 R_{*}\left(0.105 R_{*}\right)$ & $0.026 R_{*}\left(0.105 R_{*}\right)$ & $0.026 R_{*}\left(0.105 R_{*}\right)$ & $0.026 R_{*}\left(0.105 R_{*}\right)$ & $0.026 R_{*}\left(0.105 R_{*}\right)$ & $0.026 R_{*}\left(0.105 R_{*}\right)$ \\
\hline 3800 & 150 & $0.026 R_{*}\left(0.105 R_{*}\right)$ & $0.026 R_{*}\left(0.105 R_{*}\right)$ & $0.026 R_{*}\left(0.105 R_{*}\right)$ & $0.035 R_{*}\left(0.140 R_{*}\right)$ & $0.035 R_{*}\left(0.140 R_{*}\right)$ & $0.035 R_{*}\left(0.140 R_{*}\right)$ & $0.035 R_{*}\left(0.140 R_{*}\right)$ & $0.035 R_{*}\left(0.140 R_{*}\right)$ & $0.035 R_{*}\left(0.140 R_{*}\right)$ \\
\hline 3800 & 200 & $0.035 R_{*}\left(0.140 R_{*}\right)$ & $0.035 R_{*}\left(0.140 R_{*}\right)$ & $0.035 R_{*}\left(0.140 R_{*}\right)$ & $0.035 R_{*}\left(0.140 R_{*}\right)$ & $0.035 R_{*}\left(0.140 R_{*}\right)$ & $0.035 R_{*}\left(0.140 R_{*}\right)$ & $0.035 R_{*}\left(0.140 R_{*}\right)$ & $0.035 R_{*}\left(0.140 R_{*}\right)$ & $0.044 R_{*}\left(0.175 R_{*}\right)$ \\
\hline 3900 & 60 & $0.026 R_{*}\left(0.105 R_{*}\right)$ & $0.026 R_{*}\left(0.105 R_{*}\right)$ & $0.026 R_{*}\left(0.105 R_{*}\right)$ & $0.035 R_{*}\left(0.140 R_{*}\right)$ & $0.035 R_{*}\left(0.140 R_{*}\right)$ & $0.035 R_{*}\left(0.140 R_{*}\right)$ & $0.035 R_{*}\left(0.140 R_{*}\right)$ & $0.035 R_{*}\left(0.140 R_{*}\right)$ & $0.035 R_{*}\left(0.140 R_{*}\right)$ \\
\hline 3900 & 100 & $0.026 R_{*}\left(0.105 R_{*}\right)$ & $0.026 R_{*}\left(0.105 R_{*}\right)$ & $0.026 R_{*}\left(0.105 R_{*}\right)$ & $0.035 R_{*}\left(0.140 R_{*}\right)$ & $0.035 R_{*}\left(0.140 R_{*}\right)$ & $0.035 R_{*}\left(0.140 R_{*}\right)$ & $0.035 R_{*}\left(0.140 R_{*}\right)$ & $0.035 R_{*}\left(0.140 R_{*}\right)$ & $0.035 R_{*}\left(0.140 R_{*}\right)$ \\
\hline 3900 & 150 & $0.035 R_{*}\left(0.140 R_{*}\right)$ & $0.035 R_{*}\left(0.140 R_{*}\right)$ & $0.035 R_{*}\left(0.140 R_{*}\right)$ & $0.035 R_{*}\left(0.140 R_{*}\right)$ & $0.035 R_{*}\left(0.140 R_{*}\right)$ & $0.035 R_{*}\left(0.140 R_{*}\right)$ & $0.044 R_{*}\left(0.175 R_{*}\right)$ & $0.044 R_{*}\left(0.175 R_{*}\right)$ & $0.044 R_{*}\left(0.175 R_{*}\right)$ \\
\hline 3900 & 200 & $0.035 R_{*}\left(0.140 R_{*}\right)$ & $0.035 R_{*}\left(0.140 R_{*}\right)$ & $0.035 R_{*}\left(0.140 R_{*}\right)$ & $0.044 R_{*}\left(0.175 R_{*}\right)$ & $0.044 R_{*}\left(0.175 R_{*}\right)$ & $0.044 R_{*}\left(0.175 R_{*}\right)$ & $0.044 R_{*}\left(0.175 R_{*}\right)$ & $0.044 R_{*}\left(0.175 R_{*}\right)$ & $0.044 R_{*}\left(0.175 R_{*}\right)$ \\
\hline 4000 & 60 & $0.035 R_{*}\left(0.140 R_{*}\right)$ & $0.035 R_{*}\left(0.140 R_{*}\right)$ & $0.035 R_{*}\left(0.140 R_{*}\right)$ & $0.044 R_{*}\left(0.175 R_{*}\right)$ & $0.044 R_{*}\left(0.175 R_{*}\right)$ & $0.044 R_{*}\left(0.175 R_{*}\right)$ & $0.044 R_{*}\left(0.175 R_{*}\right)$ & $0.044 R_{*}\left(0.175 R_{*}\right)$ & $0.044 R_{*}\left(0.175 R_{*}\right)$ \\
\hline 4000 & 100 & $0.035 R_{*}\left(0.140 R_{*}\right)$ & $0.035 R_{*}\left(0.140 R_{*}\right)$ & $0.035 R_{*}\left(0.140 R_{*}\right)$ & $0.044 R_{*}\left(0.175 R_{*}\right)$ & $0.044 R_{*}\left(0.175 R_{*}\right)$ & $0.044 R_{*}\left(0.175 R_{*}\right)$ & $0.044 R_{*}\left(0.175 R_{*}\right)$ & $0.044 R_{*}\left(0.175 R_{*}\right)$ & $0.044 R_{*}\left(0.175 R_{*}\right)$ \\
\hline 4000 & 150 & $0.044 R_{*}\left(0.175 R_{*}\right)$ & $0.044 R_{*}\left(0.175 R_{*}\right)$ & $0.044 R_{*}\left(0.175 R_{*}\right)$ & $0.052 R_{*}\left(0.209 R_{*}\right)$ & $0.052 R_{*}\left(0.209 R_{*}\right)$ & $0.052 R_{*}\left(0.209 R_{*}\right)$ & $0.052 R_{*}\left(0.209 R_{*}\right)$ & $0.052 R_{*}\left(0.209 R_{*}\right)$ & $0.052 R_{*}\left(0.209 R_{*}\right)$ \\
\hline 4000 & 200 & $0.052 R_{*}\left(0.209 R_{*}\right)$ & $0.052 R_{*}\left(0.209 R_{*}\right)$ & $0.052 R_{*}\left(0.209 R_{*}\right)$ & $0.052 R_{*}\left(0.209 R_{*}\right)$ & $0.061 R_{*}\left(0.244 R_{*}\right)$ & $0.052 R_{*}\left(0.209 R_{*}\right)$ & $0.061 R_{*}\left(0.244 R_{*}\right)$ & $0.061 R_{*}\left(0.244 R_{*}\right)$ & $0.061 R_{*}\left(0.244 R_{*}\right)$ \\
\hline
\end{tabular}

